\title{
Swallowing the Black Pill: A Qualitative Exploration of Incel Antifeminism within Digital Society
}

\author{
By \\ Angus Charles Lindsay \\ Supervisor: Professor Jan Jordan \\ Co-Supervisor: Dr Carol Harrington
}

\begin{abstract}
A thesis submitted to Victoria University of Wellington in fulfilment of the requirements for the degree of Master of Arts in Criminology
\end{abstract}

Social and Cultural Studies

Victoria University of Wellington

2020 


\section{Acknowledgements}

To those who said I could do it, but even more so to those who said I could not.

I'd like to acknowledge those individuals whose comments were analysed in the production of this thesis. In this study of your subculture, I can almost guarantee you will not like some of what you will read. However, I did my best to carve out a critical understanding of your world and the pain you experience. This thesis was inspired by the adage "Hurt people, hurt people." I do not think that you are individually bad, evil or morally contempt. I wish you all find peace and love in your journey.

I would like to acknowledge the victims of violence. In particular, those who have passed at the hands of cultures which encourage militant misogyny, of which this thesis is primarily concerned. I would also like to acknowledge their families, friends and communities who continue to deal with the blight of male-violence every day.

I wish to acknowledge my supervisors (including my unofficial supervisors) who I have leaned on greatly to create a thesis (hopefully) worth reading. I also want to acknowledge the many informal meetings, elevator discussions and impromptu coffees with the lecturers and postgrads in the School of Social and Cultural Studies - your comments, feedback and support have been greatly appreciated in conducting this interdisciplinary work.

I particularly want to thank the School of Criminology and the admin staff on level 9; you have backed me entirely throughout my university career and every one of you has pushed me in different ways. You have helped to shape and inform my critical view of the world and I will be forever grateful for that.

My proofreaders: sorry, just sorry.

To my family. When I completed my undergraduate degree, I was the first in my immediate family to go to university. Without you, this would not have been possible. I hope that I have made you proud.

To my partner, thank you for putting up with my grumpiness, my impecuniousness and my constant need for validation while researching this dark topic. Thank you for your rationality and calculatedness, they have certainly helped me throughout the last few years at university.

Finally, I am grateful to the Victoria University of Wellington's Master's by Thesis scholarship which provided me with financial support for the duration of this thesis. 


\begin{abstract}
This thesis explores the online 'manosphere' subculture of Involuntary Celibates (Incels). Incels have been widely discussed in contemporary media in recent years and have been cited as harmful after several mass-murders and attacks have taken place offline. Previous academic research has largely focused on individual-level explanations for Incel mass-murderers, with few studies seeking to uncover the structural determinants of the rise of Incels. This thesis attempts to fill this gap, exploring the subculture's negotiation with the changing features of contemporary society. The study utilised internet-based qualitative research methods over a period of three-months to collect data on two Incel forums: r/Braincels and Incels.co. The data was then interpreted through thematic analysis within a constructivist grounded theory approach. The research found that Incels negotiate their anxieties of a rapidly changing globalised world with a sense of victimisation and 'aggrieved entitlement' through a worldview that understands society as set up to economically, socially, and sexually favour women. It was also found that through such a sense of entitlement, Incels conceive of a hetero-patriarchal racial caste-system that relies on uncritical readings of selected biological and evolutionary psychological studies. This worldview is known as the 'Black Pill' and is employed to ideologically condition Incels against out-groups. Through a shared mythology of victimisation, the Incel ideology of the Black Pill functions to produce a form of 'stochastic terrorism' in which individual users interpret the spectrum of beliefs from enacting online gender-based hate-speech to mass violence in the terrestrial world. This thesis presents understandings that could inform future educational programs in critical literacy skills that aim to dismantle the conceptual apparatus that feeds the ideologically charged hatred of groups like Incels.
\end{abstract}




\section{Contents}

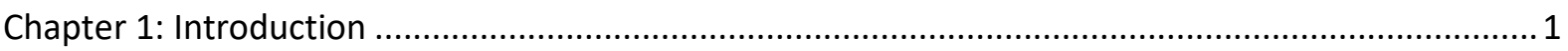

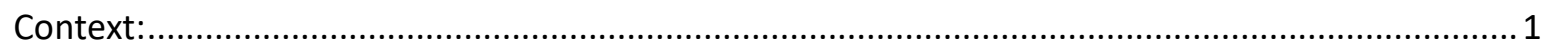

Thesis Overview:

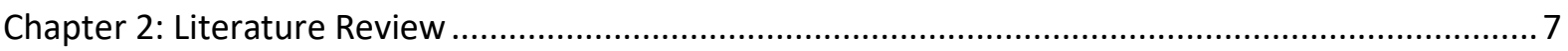

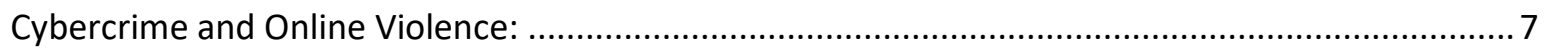

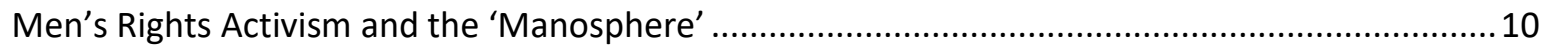

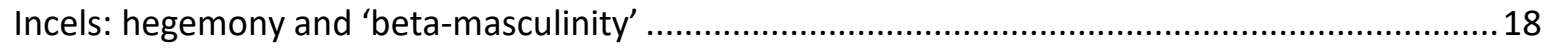

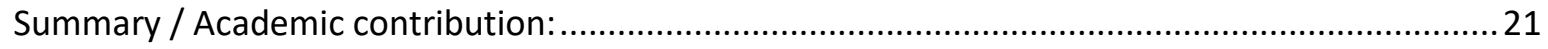

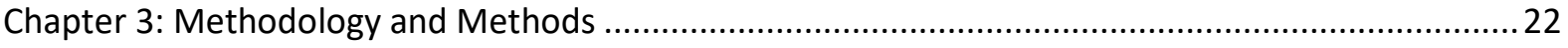

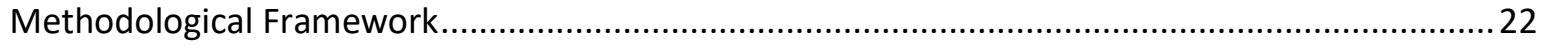

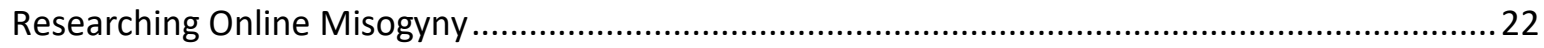

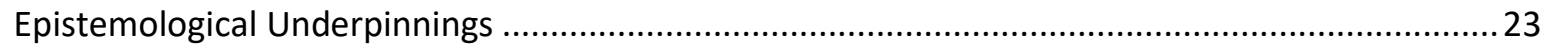

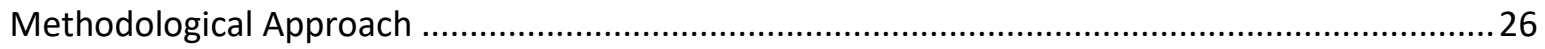

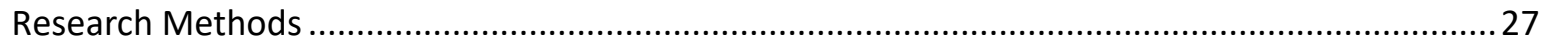

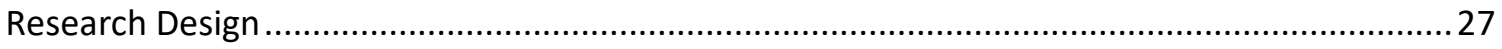

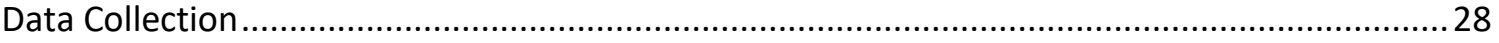

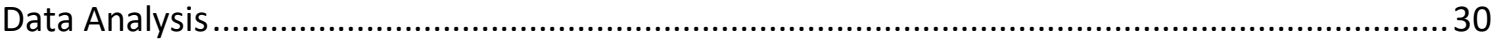

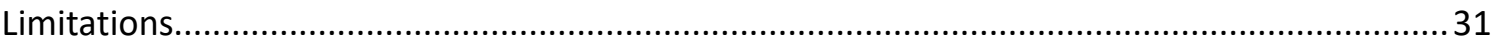

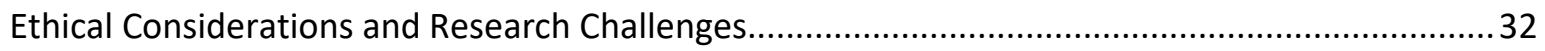

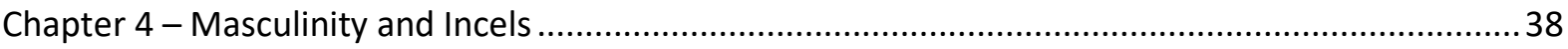

Ideals of Hegemonic Masculinity: Painting the picture of 'Chad Thundercock' ............................... 38

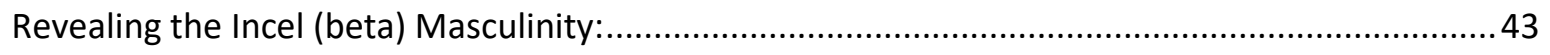

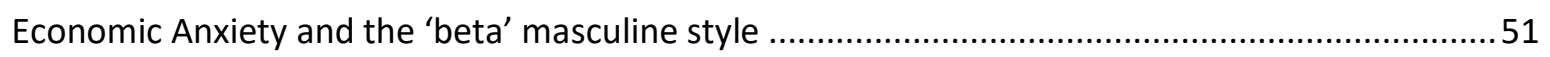

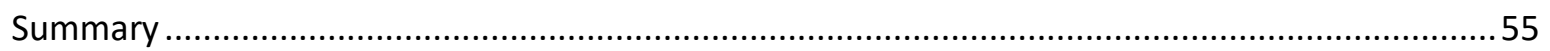

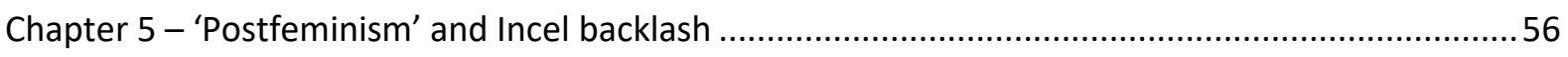

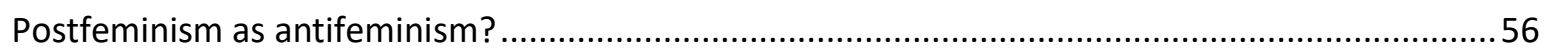

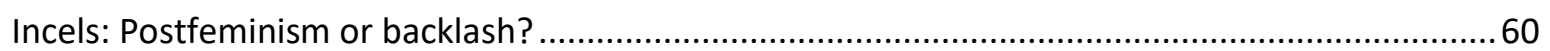

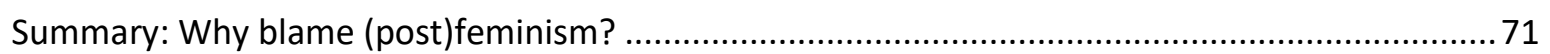



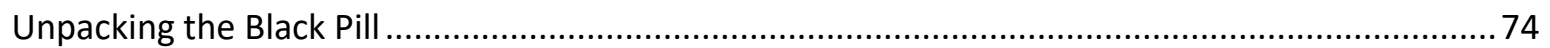

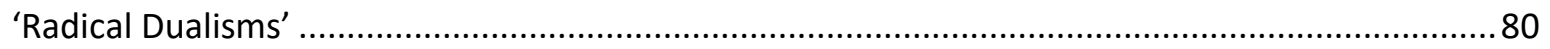

Normalisation of ideologically structured hatred against women ............................................... 83

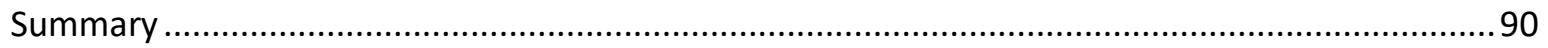

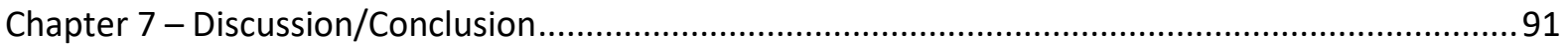

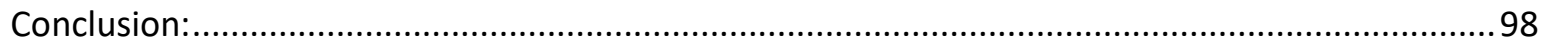


Appendix.

101

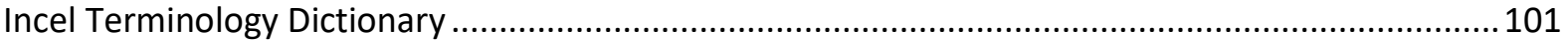

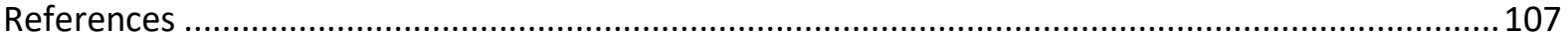




\section{Chapter 1: Introduction}

\section{Context:}

Violence against women is not a new phenomenon. The United Nations' Declaration on the Elimination of Violence Against Women states that "violence against women is a manifestation of historically unequal power relations between men and women", and that "violence against women is one of the crucial social mechanisms by which women are forced into a subordinate position compared with men" (United Nations, 2014, para. 7). In an increasingly interconnected digital age, understanding the role of the 'social' in technology use and the role of technology in facilitating violence becomes imperative (Powell, Stratton, \& Cameron, 2019; Segrave \& Vitis, 2017). In the Web 2.0 period there has been a growing awareness of the relative ease, anonymity, and reach of online technologies, which has prompted questions about our negotiation with online platforms. ${ }^{1}$ Critical reflections on digital technologies have emerged which recognise the necessity of challenging the relationship between social hierarchies, gender relations, power structures and the role and use of communicative media (Fuchs, 2014). The evolving nature of communicative technologies in the late twentieth and early twenty-first centuries prompted a considerable shift in academic attention relating to interpersonal violence within the technosocial landscape. In particular, ongoing feminist challenges in revealing the invisibility, acceptability and privatisation of issues surrounding gendered violence have driven social change (Henry \& Powell, 2016; Powell \& Henry, 2019). However, the scope and scale of violence against women and children is still present despite gains made in policy reform and equality, and there are now expanding risks and opportunities for crime to occur with the ubiquity of new digital technologies in everyday life (Grabosky, 2001; Yar, 2012).

During the contemporary digital era of communicative and collaborative media, technosocial networks have proliferated. The Web 2.0 marked a shift from the one-to-many logic of the Web 1.0 era of the internet towards that of an increasingly communicative and collaborative space, with a host of platforms that facilitated user-generated content development and sharing, as well as social networking. Since 2002, there has been an explosion of social networks and

\footnotetext{
${ }^{1}$ A term which refers to the advent of online technologies such as Wi-Fi, broadband access, wireless computing etc., that enable interactive capabilities in an environment characterised by user control, freedom, and dialogue (Lupton, 2015).
} 
image-sharing platforms, including MySpace, Facebook, Youtube, Twitter, Tumblr, Instagram and Reddit. A growing focus of contemporary cybercrime research has thus been to examine the nature and patterns of criminal online social networks (Brewer, 2017; Décary-Hétu, \& Dupont, 2012; Westlake \& Bouchard, 2016). With the rapid uptake in the use of internetenabled devices and online communication services such as social media networks and social applications, it has been shown that digital technologies are being used as tools to facilitate harms against women (Henry \& Powell, 2015; 2016). Global estimates published from the World Health Organisation (2014) show that 35\% of women worldwide report having experienced either physical or sexual violence. Recent studies of online behaviours further demonstrate that these harms are commonplace and not merely confined to the 'offline' world (Citron, 2014; Oksanen, 2014; Powell \& Henry, 2019).

In the \#MeToo era, conversations about misogynistic attitudes, harassment and violence against women have increasingly been at the forefront of public debate. ${ }^{2}$ Research has shown that in the digital sphere, misogynistic attitudes underpin much of the harassment experienced online, with women being the primary targets and men being the likely perpetrators (BanetWeiser, 2016; Citron, 2012; Jane, 2014). Many of the online harms in the digital era are associated with the technosocial networks of the manosphere, which are understood as the digital manifestation of the Men's Rights Movement (Ging, 2017; Jones, Trott, \& Wright, 2019). These nefariously antifeminist networks have been shown to coalesce with one another and proliferate in the form of 'harmless' memes, jokes and other cultural material, as well as more damaging forms of online attacks such as doxxing, trolling, and hacking to achieve political action and personal retribution (Ging, 2017; 2019; Lumsden, 2019). ${ }^{3}$ This vile antifeminism has bled out into offline spaces, as in the case of Incel (involuntary celibate) mass murder. In 2014, 22-year-old Elliot Rodger, who was deeply immersed in the manosphere, killed six people and injured 14 others in Isla Vista, California. Rodger's mass-murder drew media attention to the Incel subculture and particularly the misogyny and glorification of violence present in online Incel communities. It was clear from an analysis of Rodger's lengthy manifesto that his killing spree was an attempt to punish young women for their disinterest in

\footnotetext{
${ }^{2}$ The \#MeToo movement is an international social justice and empowerment movement that began online in 2006 and is based upon breaking the silence of sexual harassment survivors. The movement came to the fore in 2017 when the New York Times ran its piece outing Harvey Weinstein for sexual assaults, of which he was convicted in February 2020. It is adopted online as a tactic to empower women through empathy and strength in numbers by visibly demonstrating the numbers of women who have survived sexual assault and harassment, especially in the workplace.

${ }^{3}$ Doxing refers to search for and publish private or identifying information about (a particular individual) on the Internet, typically with malicious intent.
} 
him and young men for their perceived sexual success (Vito, Admire, \& Hughes, 2017). Frustrated with his lack of social and sexual success, Rodger sought comfort in the manosphere communities who shared and commiserated about their ability to 'pick up' women. Rodger and other mass-murderers such as the 2018 Toronto Van Attack perpetrator, 25-year-old Alek Minassian were applauded by members of online Incel communities on Reddit, 4Chan and other Incel-related online spaces (Jaki et al., 2019).

During the course of this thesis, several so-called "Incel-related" attacks have taken place which have garnered significant traction in international media. Two confirmed examples of Incel-related attacks took place in North America, however, many online media outlets have been quick to jump to label other lone-wolf shooters as Incels, such as the 2019 Dayton, Ohio and El Paso, Texas mass-shooters and others who possess a common thread of being lonely, angry white men who share a hatred for women (Danner \& Raymond, 2019; Follman, 2019). ${ }^{4}$ In Texas, Incels have been labelled an "emerging domestic terrorism threat" (Texas Department of Public Safety, 2020 p.3). Todd Phillips' 2019 movie Joker also received mainstream critique for its potential to incite Incel-related violence, and even warranted warnings from the U.S military over potential violence at screenings of the film (Bharti, 2019). Recognising the connection between such ideologies and violence that can proliferate in online echo chambers, digital platforms such as Reddit have begun to respond to the threat posed by radical manosphere groups, including closing the r/Incels and r/Braincels subreddits.

Finally, within the course of this study, Aotearoa/New Zealand experienced its most devastating mass shooting. On 15 May 2019, an Australian-national entered several mosques in Christchurch, killing 51 people and injuring 49 others. Although the Christchurch shooting was not specifically Incel-related, the cross-pollination of ideas, norms and values between manosphere groups has become undeniable (Ging, 2017). It is within an international and domestic context in which the growing phenomenon of Incels and their noted interconnections with radical extremist organisations such as Alt-Right and White Supremacist groups must be further interrogated in order to prevent such expressions of mass-violence, and to shed light on the contexts in which they derive.

Research Aims and Significance:

\footnotetext{
${ }^{4}$ For example, Brian Clyde, June 2019, Dallas, Texas, and Alexander Stavropoulos, January 2020, Sudbury, Ontario are both confirmed examples of attackers who admitted to being Incels or utilising Incel-related ideology.
} 
This thesis was guided by three overarching aims:

1. To explore and describe the Incel subculture by examining the discourse, memes and other cultural material specifically forged and (re)produced through Incel forums and networks

2. To gain insights and critically examine Incels' conceptualisation and negotiation with hegemonic masculine norms.

3. To understand how online technologies and cultures are weaponised through technosocial networks.

The experiences of the men observed in these international forums have direct implications for men living in Aotearoa/New Zealand. The structural features of a globalised, neoliberal and so-called 'postfeminist' society interact with the technological affordances of social media to create a space in which some men view society as economically, sexually and socially favouring women. This has led to a sense of 'aggrieved entitlement' and victimisation (Kimmel, 2014). This victimisation is argued by Incels to be at the hands of feminist social progress. Their calls for the restriction of women's rights and the celebration of a (White) masculine utopia in which lies a racialized and patriarchal hierarchy are noted throughout the thesis. Incels ideologically structured violence and misogyny is shown to be facilitated by a set of biological and evolutionary psychological theories and studies which, when combined, are referred to as the 'Black Pill.' Such insights into the ideological conditioning that takes place within such forums also extends to wider 'masculine' internet cultures within the manosphere space, and in other online communities that harbour such self-described 'beta' males.

This research will also have implications for policy, research, and violence prevention initiatives. It is vital to understand the developments in the mechanisms through which individuals' worldviews become shaped and influenced by pervasive influences that lead some young men down a path of violence. The insights gathered provide support to organisations dedicated to teaching critical literacy skills, sexual violence prevention and progressive views of gender that work to dismantle the conceptual apparatuses that feed the ideologically charged hatred of groups like Incels. The thesis also should compel governments to mitigate the harms of an unequal society by taking gender-related violence more seriously. It is anticipated that this research will assist in furthering academic understanding of the interconnections between Incel, Alt-Right, and other manosphere cultures. 


\section{Thesis Overview:}

This thesis presents qualitative data derived from a three-month observational study of two online Incel forums. The aim of this research is to explore the virulent misogyny espoused by one subcultural group of the wider manosphere, namely Incels. Incels occupy a noteworthy position within the proliferation of groups within the manosphere, as their expressions of antifeminism and racism are among the most violent and hate-filled. As will be discussed in the literature review (Chapter Two), much of the Incel-related research that has preceded this thesis has focused on individual or micro-level explanations for Incel-related violence. Therefore, my focus on the structural and ideological particularities of the group provides an important contribution to the burgeoning field of academic knowledge about Incels. In Chapter Three, I discuss my methodological decisions, ethical conundrums, and problems regarding researching online hate-groups. I also reflexively examine my personal experiences as a qualitative internet researcher and illuminate some of the challenges I encountered doing this research.

Chapters Four and Five utilise thematic data to interrogate the structural origins of the Incel subculture. To 'unpack' the Incel masculinity, I sketch a caricaturised version of Incels' understanding of the dominant form of masculinity. I then show how their self-positioning as 'beta males', and their dissent against dominant norms of masculinity, work to reinforce a 'crisis of masculinity'. This crisis-narrative works to justify attacks on other non-traditional performances of masculinity and women through virulent online misogyny, and occasionally extreme expressions of violence. I then comment on how economic anxieties reify such crisis narratives. I further expand these structural arguments in Chapter Five to examine how the social and cultural context of postfeminism impacts on masculine subjectivities and men's practices (O’Neill, 2015). I evaluate Incels' relationship to feminism and argue that the neoliberalisation of gender politics (Gill, 2017) has resulted in backlash against perceived feminist gains. The contemporary digital politics observed through this study reveals a confluence of misogynist and white supremacist worldviews. Through such structural particularities, I demonstrate how Incels collective venting of anger centres on a defining paradox: that (White) men hold institutional power in patriarchal society, but most do not feel that they possess such power.

In Chapter Six I reveal insights gained about the 'Black Pill' ideology and suggest that this array of theories, which over-subscribes to genetic determinist philosophies and cherry-picked 
studies, acts as an explanatory mechanism for Incels' perceived alienated positions within society. The Black Pill is used to justify their perceived oppression by women through the assertion of biological theories that purport to highlight the so-called 'immutable' traits of social and sexual exclusion. I show that the Black Pill also assists in creating a shared language, culture and social identity that gives Incels a sense of significance and glory, which is co-opted, but distinct from the wider manosphere. I suggest that this ideology provides the justification for wholesale misogyny, calls for a re-ordering of society, and extreme violence against outgroups. It is thus considered to be an ideological tool utilised to radicalise forum members. Chapter Six expands on this discussion, highlighting the implications for the ideological belief in the Black Pill, which broadens previous discussions on radicalisation into the wider context of violence against women. In Chapters Six and Seven I briefly problematize the labelling of groups such as Incels as 'terrorist' groups, however, I argue that Incels' ideology is dangerous insofar as it produces the justification for individuals to commit 'stochastic terrorism'. Finally, Chapter Seven concludes with reflections on future directions for Incel-related study. 


\section{Chapter 2: Literature Review Cybercrime and Online Violence:}

The pre-web era of the 1980s to 1991 saw government institutions, education providers and corporations adopt personal computers in their daily activities. This saw a greater reliance on electronic data storage and increased connectivity within closed and private networks (Ceruzzi, 2003). Criminology within the pre-web era began to recognise that the widespread use of personal computers left governments, corporations and educational institutions vulnerable to new forms of crime through technology misuse. Computer-related crime concerns of the time were often practices that have long existed, but are altered or amplified via new technologies. Economic crimes, data theft, identity fraud and the security and privacy of information systems were major concerns of the time (Clough \& Mungo, 1992; Yar, 2012). Initially, due to government and corporate institutions adopting computer-related technologies, many of the emerging harms were mostly associated with white-collar crime (Croall, 1992).

The public release of the internet, the World Wide Web, went live across the world on 6 August 1991. The Web 1.0 or the 'global web' era (1991-2000s) invoked an associated shift in research on the internet and cybercrime. Criminologists recognised that the increased accessibility of online information sharing and communication on a global scale created new and evolving opportunities for crime against individuals, corporations and institutions. The field began to question threats around national security, cyber security and terrorism that were now changing with the advent of the internet (Yar, 2013). While the original concerns that are associated with white collar and financial crimes remained persistent avenues of inquiry, the attention of cybercrime scholars quickly broadened to engage with interpersonal harms such as online child sexual exploitation, human trafficking and child pornography (Powell, et al., 2018; Yar, 2013). These crimes sparked the attention of much public and policy concern, and encouraged the need for analysis in order to understand this new online frontier of crime and to identify when, where and how criminal justice systems ought to respond.

Through the development of Web 2.0 and the 'social web' (2000s to present), criminology saw a shift in cybercrime research. The Web 2.0 marked a shift from the one-to-many logic of the Web 1.0 era of the internet towards that of an increasingly communicative and collaborative space, with a host of platforms that facilitated user-generated content development and sharing, and social networking. Between 2002 and 2010, there was an explosion of social networks and 
image-sharing platforms, including MySpace, Facebook, Youtube, Twitter, Tumblr, Instagram and Reddit. Contemporary cybercrime research has grown to focus on identifying and understanding the nature and patterns of criminal online social networks (Décary-Hétu \& Dupont, 2012; Westlake \& Bouchard, 2016). A further feature of the social web era is the proliferation of smartphones, social media and wearable technology that is simultaneously collecting expansive 'big-data' about our identities, ourselves and our everyday lives (Powell et al., 2018; Segrave \& Vitis, 2017). Criminologists have also begun to engage with the increasingly algorithmic and computational capacities in relation to crime data analytics, law enforcement and justice system practices (Brantingham, 2011; Chan \& Bennett-Moses, 2016). ${ }^{5}$

These new developments have prompted further questions in understanding the 'social' in technology use and the role of technology in new forms of technological violence (Powell et al., 2018; Segrave and Vitis, 2017). Research into cyberbullying, cyberstalking and online harassment rapidly expanded over the Web 2.0 period as the relative ease, anonymity and reach of online communications was associated with continuing concerns regarding invasive and threatening communications (Reyns, Henson \& Fisher, 2011; Spitzberg \& Hoobler, 2002). Critical reflections on digital technologies have emerged which recognise the necessity of challenging the relationship between social hierarchies, gender relations, power structures and the role and use of communicative media (Fuchs, 2014). For example, Yar (2012) argues that if we understand the internet not as a technology, but as a shifting set of social practices, we can begin to grasp how changes to the online environment can reshape patterns of crime and victimisation.

The evolving nature of communicative technologies in the late twentieth and early twenty-first centuries prompted a considerable shift in academic attention relating to interpersonal violence within the technosocial landscape. This has been particularly evident in ongoing feminist challenges in revealing the invisibility, acceptability and privatisation of issues surrounding gendered violence which has driven social change (Henry \& Powell, 2015; 2016; Powell \& Henry, 2019). However, the scope and scale of violence against women and children persists, and despite gains made in policy reform and equality, there are new risks and opportunities for crime to proliferate with the ubiquity of new digital technologies in everyday life (Grabosky, 2001; Yar, 2012). There is also growing media, activist, practitioner, legal and scholarly attention to technologically facilitated violence, where mobile and online technologies are

\footnotetext{
${ }^{5}$ Critical criminologists in particular have been critical of the computational and algorithmic politics of such technology in relation to criminal justice and mass-surveillance (see: McCulloch \& Wilson, 2015).
} 
being used as tools to blackmail, control, coerce, harass, humiliate, objectify or violate (Henry \& Powell, 2015; 2016; Powell \& Henry, 2019).

The lines identifying internet-facilitated abuse are often blurry and uncertain when constant digital presence and communications are normative (Maher et al., 2017). When such normative boundaries are crossed, digital technologies can be weaponised and used as tools to facilitate violence and harassment. Powell and Henry (2019) examined the prevalence and nature of technology-facilitated sexual violence (TFSV) among a sample of 2,956 Australian adults. The study examined multiple dimensions of non-consensual sexual behaviours toward adult victims facilitated wholly or partially via digital communications technologies. Influenced by Brown's (2006) call for social science to look beyond the distinct offline/online dualism that studies of cybercrime and cyberharm often perpetuate, Powell and Henry (2019) argue that "contemporary manifestations of sexual violence are increasingly and variously mediated, facilitated, or extended via the internet and other digital communications and technologies, including the use of images and social media” (p. 3). Importantly, Powell and Henry's (2019) study purposely utilises the concept of TFSV in relation to deliberately constructed "sexed" and "gendered" subjects (Connell, 1987). This reflects the understanding that sexual violence in digital forms is experienced in gendered ways by male and female victims, and a gendered focus serves to remind us that TFSV is experienced by all genders.

At present, few studies illustrate the empirical experiences of either gender or sexuality-based harassment in the digital world. Some scholars have discussed the nature and potential impacts of gender and/or sexuality-based harassment in online spaces (Lewis, Rowe, \& Wiper, 2019; Powell \& Henry, 2019), noting the likely effect of creating hostile and exclusionary spaces for women, as well as for gender and sexuality-diverse groups. Oksanen et al. (2014) discovered that of 723 Finnish Facebook users, 67\% had been disparaged online, most of which (68\%) was focused on the individual's sexual orientation. Interestingly, there appeared to be no significant differences found between genders or by age. Citron (2014) has noted that male victims of online harassment often experience sexualised harassment involving attacks on their gender, sexuality, or masculinity. This suggests men and women experience online hate in different ways. In the most recent empirical research, Powell and Henry (2019) highlight that women report significantly higher rates of digital sexual harassment, whilst men report higher rates of other online victimisation for other categories including nude or semi-nude images taken or sent onto others without permission, and images taken of an unwanted sexual experience. In particular, men's victimisation results in disparaging comments about their 
sexuality or sexual identity. Non-heterosexual identifying respondents were significantly more likely than heterosexual identifying respondents to report online sexual harassment, as well as both gender and sexuality-based harassment. When discussed in relation to Oksanen et al's (2014) finding, this is unsurprising, as sexuality-based harassment constitutes a large portion of young people's exposure to online hate.

Previous research has examined rape threats (Jane, 2014), death threats (Megarry, 2014) and cyber-stalking (Pittaro, 2007) as examples of behaviours that are used to attack and silence women in online spaces. However, Jones, Trott, and Wright's (2019) study found a high degree of passive harassment in their study of the Men Going Their Own Way (MGTOW) forum, and demonstrated the banality of the misogyny and sexism reflected in the group's rhetoric. Manne (2017) highlighted a difference between sexism and misogyny, arguing that sexism is the ideology that supports patriarchy, while misogyny polices it. According to Manne (2017) misogyny is a response to the threat of feminism to the patriarchy and operates as a way of punishing those who step beyond the status-quo. Manne's (2017) understanding of this difference allows us to highlight the different purposes and intensions of sexism and misogyny, whether that be the passive harassment found in Jones et al., 2019, or the more violent coercion, objectification, and violence present in other studies (Ging, 2017; 2019; Henry \& Powell, 2016; Jane, 2014; Megarry, 2014; Piattro, 2007; Powell \& Henry, 2019).

\section{Men's Rights Activism and the 'Manosphere'}

The troubling rise in the new 'toxic technocultures' (Massanari, 2015) such as Incels', 'Men's Right's' and other forms of exclusionary politics have been explained to be driven by economic insecurity (Inglehart, 2016; Kiely \& Saull, 2017; Messner, 2016). Several studies have shown that sexism, racism and economic dissatisfaction were strong correlates to the rise of the extreme right (Schaffner, MacWilliams \& Netea, 2017; Wayne, Ocean, \& Valentino). Green (2019) highlights that internet forums provide "a fertile ground for the coalescence and growth of new manifestations of extreme-right communities" (p. 65). While this research does not examine the Alt-Right in particular, it highlights that the misogynist and racist politics that embody the movement have developed alongside a 'backlash' against feminist action and social justice movements of the past 30-years, while socio-economic changes to the working class have been largely ignored (Green, 2019; Odin Shaw, 2019). Previous studies have established the broader manosphere, which both Incels and the Alt-Right are constituents of (Ging, 2017; 2019), which share in the 'politics of sentiment' of a crisis of (White) masculinity. 
Therefore, such research is an establishing link between misogynist attitudes and economic anxiety (Odin Shaw, 2019).

The economic and cultural changes brought in by post-Fordism globalization and neoliberalism challenged the primacy of the White male worker in the Global North. In particular, the 1980s recession accelerated the deindustrialization of the American labour force. This resulted in the loss of millions of unionized jobs, rising levels of structural unemployment, and the growth of low-paid non-unionized service sector jobs (Messner, 2016). Other global north countries such as the UK, Australia, Canada and Aotearoa/New Zealand also facilitated this economic restructuring by removing many of the previously set economic safety nets and adopting a neoliberal system that celebrated individualism and market primacy, while slashing taxes on rich individuals and corporations, alongside cutting support for welfare and education (McDowell, 2019). Such shifts have continued throughout the current era, resulting in the dramatic growth of a super-rich minority, a shrinking middle-class and record numbers of working poor in the population as rates of inequality and social deprivation skyrocket. For many men, the assured job stability that was available to their fathers was replaced with casual and fluid work situations and growing levels of unemployment (Kimmel, 2015).

The shift to post-Fordism and neoliberalism saw the search for cheap foreign labour and a growing 'feminization' of the workforce. Such changes challenged the economic privilege which men could have expected in the mid-to-late $20^{\text {th }}$ century, as "under neoliberalism, the breadwinners of the global north are a dying breed" (Salzinger, 2016, p. 9). As economic restructuring accelerated from the 1980s and 1990s, globalization shifted many factory jobs to countries rich with vast pools of cheap labour in the global south (Salzinger, 2016). These flights of capital were compounded by the impacts of women's increased entry into the labour market - borne as much out of necessity as for reasons sparked by the ideals of feminist empowerment. More highly educated women entered into a growing field of white-collar occupations, while the greater mass of women filled an expanding array of low-paid pink collar and service sector jobs (Charles \& Grusky, 2004). During this economic restructuring, the professional or white-collar class of men continued to fare reasonably well, however, blue collar and poor men faced an increasingly bleak economic environment (Kimmel, 2015; Messner, 2016).

During these economically turbulent times, the late 1970s and 1980s saw a proliferation of Men's Right's Activism (henceforth MRA) groups who gained leverage among men who felt 
oppressed by society and blamed women (and feminism) for their supposed decline in power (Kimmel, 2017; Schmitz \& Kazyack, 2016; Whitehead, 2002). Such structural changes created a feeling of 'crisis' amongst men, who, for a disproportionately large portion of young, bluecollar men, were made redundant by economic deregulation and a changing workforce. Authors such as Kimmel $(2011 ; 2017)$ and Messner (2016) recognised that for many lower and middle-class men, such changes have undermined their identities and sense of masculinity, which were attached to employment, success and the breadwinner status. Some men responded to their declining status within society with fear and powerlessness, which Kimmel (2010) refers to as 'aggrieved entitlement'; a state where men believe "themselves to be entitled to power - by a combination of historical legacy, religious fiat, biological destiny, and moral legitimacy" (p. 148).

The origins of the contemporary MRA movement lie in response to the 'second wave' of feminism during the 1960s and 1970s. Some MRA leaders promoted the idea of a 'men's liberation movement' that would work in symmetry with feminism to bring about progressive personal and societal change (Messner, 1998; 2016). Coinciding with an outgrowth of feminist research on gender, in particular the language of sex roles, men's liberationists argued that as the 'female sex role' and patriarchy had clearly oppressed women, 'the male sex role' also had negative effects for men (Marwick \& Caplan, 2018; Messner, 2016). Early men's liberationists discussed issues that negatively impacted men such as emotional stoicism, unequal child support obligations, male-only draft requirements, and the social pressures of traditional masculinity (Coston \& Kimmel, 2012; Kimmel, 2017; Messner, 2016). Messner (1998) noted that early men's liberationists sought to "attract men to feminism by constructing a discourse that stressed how the male role was impoverished, unhealthy, and even lethal for men" (p. 256).

However, despite promising beginnings of a movement that could have worked symmetrically with feminism, navigating the tensions between emphasising male privilege and the costs of masculinity proved too difficult (Messner, 2016). By the late 1970s to the early 1980s, the men's liberation movement split off into separate pro- and anti-feminist factions. Anti-feminist MRAs took advantage of shifting gender norms, and a society changing by the sexual and cultural revolutions of the $60 \mathrm{~s}$ and $70 \mathrm{~s}$, to gain leverage among men who felt oppressed by society and blamed women (and feminism) for their supposed decline in power (Kimmel, 2015; Schmitz \& Kazyack, 2016; Whitehead, 2002). 
During the 1980s-2000s, MRA's became largely concerned with state-centred issues, particularly feminism's perceived attack on fatherhood through family law (Gotell \& Duton, 2016; Kimmel, 2015; Maddison, 1999). MRA groups sought to challenge what they considered to be unfair law reform and policy discourse surrounding child custody, access and support (Boyd, 2004; Kimmel, 2015). The MRA movements of this period contested feminist research which highlighted the gendered character of domestic violence as well as challenging antiviolence policies, claiming discrimination against men (Dragiewicz, 2008; 2011). Advocates sought to reassert their perceived entitlement within society not by advocating for survivors of domestic violence (both male or female, and thus aligning themselves as feminist allies), but by calling for the disestablishment of domestic violence services that protect women, as well as other policies that imbue women with perceived social advantage (Dragiewicz, 2008; 2011; Kimmel, 2017; Schmitz \& Kazyack, 2016). MRA's of the Pre-Web or Web 1.0 era typically organised and fought against perceived attacks on the family and rallied in opposition to unfair treatment in divorce proceedings.

In the Social Web or Web 2.0 era, a growing body of academic literature points to the expanded capabilities of the internet for men's rights activists to publicly spread antifeminist discourse (Ging, 2017; Gotell \& Dutton, 2018; Lilly, 2016; Marwick \& Caplan, 2018; Schmitz \& Kazyack, 2016). Mirroring developments of third-wave feminism which centre on grassroots action and cultural struggles, contemporary MRA groups and websites now appear to be focused more on shifting attitudes through cyber activism, rather than influencing law and public policy (Gotell \& Dutton, 2018). MRA's in the digital age also appear to particularly focus on framing men as victims of false rape complaints, and casting rape culture as a feministinspired moral panic (Gottel \& Dutton, 2018). Messner (2016) asserts that the true threat to equality and feminist progress is posed by a "kinder, gentler variety of men's rights, taking the form of a neoliberal, professionally institutionalised 'moderate' men's rights strategy that skirts analysis of structural inequalities in favour of a common-sense celebration of individual choice for women and men" (p.16). However, Ging (2017) argues that Messner overlooks the venomous and pervasive nature of the contemporary online antifeminism seen within the manosphere. This 'new' men's rights activism has been shaped by, and is rooted within, the hegemonic structures of neoliberal capitalism and postfeminism, as well as the technological affordances of the Web 2.0 era (Ging, 2019). Such activism is now rooted within personal narratives and concerns that centre chiefly on sexual rejection and pseudo-scientific explanations of the sexual marketplaces, rather than an organised political movement. 
The manosphere is understood as a loosely connected online hub for 'men's issues.' It refers to a myriad of interconnected groups, including MRAs, pickup artists, MGTOW (Men Going their Own Way), Incels (involuntary celibates), father's rights activists and other male-centric communities and subcultures within the online-sphere (Ging, 2017; Marwick \& Caplan, 2018; Nagle, 2015). Jaclyn Friedman provides a helpful description of the manosphere:

Comment threads and message boards serve as the public square for MRAs, a kind of bizarre-world combination of locker room, group therapy, and organizing. Recently, on the Men's Rights subreddit, one MRA complained of how much he had to pay in child support, and how trapped he felt by the situation. His fellow MRAs helpfully suggested that the solution to his problem was to murder his wife, a tactic many on the subreddit were eager to echo, upvote, and get disturbingly specific about. Blogs like AVFM [A Voice for Men] and The Spearhead serve as what passes for the "think tanks" for MRAs, developing and promoting the MRA agenda. (2013, para. 11).

The manosphere is united by an antipathy towards feminism and the production of 'hard-core' misogynist discourses of gendered subjugation and violence. This movement shares the central belief that feminist values pervade society, and that men must rail against such an overreaching, misandrist culture to protect their already oppressed existence (Ging, 2017; Jones, et al., 2019; Lilly, 2016; Marwick \& Caplan, 2018; Schmitz \& Kazyack, 2016). The key concept that underpins the interconnected, yet nebulous organisations that inhabit the manosphere is that of the 'Red Pill'. The 'Red Pill' is an analogy appropriated from a scene in the 1999 film The Matrix, in which Laurence Fishburne's Morpheus offers Keanu Reeves's Neo a choice between two pills. Taking the blue pill means switching off and continuing to live a life of delusion; taking the red pill means becoming enlightened. In this case, the red pill represents awakening men to feminisms' misandry and brainwashing, the supposed 'real' enemy causing a 'crisis of masculinity' (Ging, 2017). However, as Lilly (2016) noted, the degree to which the manosphere has interaction with offline men's rights groups is unclear, aside from when manosphere sites write about or are sympathetic to campaigns or events of men's rights groups. It is clear, however, that MRA groups and the manosphere each have decidedly antifeminist and antiwomen philosophical underpinnings.

The Red Pill concept has also transcended antifeminist MRA politics. In their study of pathways to the Alt-Right, the Southern Poverty Law Centre (SPLC) examined how people came to be influenced by Alt-Right websites (Hankes \& Amend, 2018). It was identified that 
4chan and 'chan culture' were a significant influence in the rise of both the far-right and 'radicalisation'. ${ }^{6}$ Almost $23 \%$ of respondents to the SPLC study referred to 'chan' culture and the anonymous and 'politically incorrect' 4chan /pol/ message board which traffics in humour and transgression, and operates away from mainstream scrutiny (Wendling, 2018). 4chan and connected webpages have been integral in nurturing Alt-Right and other misogynist and racist groups within the manosphere (Reitman, 2018; Munn, 2019). Reitman (2018) noted how discussion threads on white supremacist websites considered how /pol/ might be utilised to help young people become "racially aware", in an example of 'red-pilling'. Similarly, Winter (2019) provided a useful review of the history, development and contemporary manifestations of the American far-right online, and demonstrates that in relation to political and technological change in the contemporary era, the neo-fascist Alt-Right has utilised new forms of technology, culture and online forms of humour to communicate and politically organise, as well as espouse racist and sexist forms of 'online othering'. Munn's (2019) article demonstrated a three-step process of gradual online radicalisation into the Alt-Right through such forms of digital communication. Such cultural and technological developments hold great influence over, and intersect with, the antifeminist politics of the manosphere and Alt-Right ideology, which clearly shows that such groups are intimately connected (Harmer \& Lumsden, 2019; Munn, 2019).

Analyses of the manosphere have shown a deluge of antifeminist ideas, divisive gender politics and feminist perspectives have been at the forefront of problematising such ideas throughout manosphere spaces (Ging, 2017; Lilly, 2016; Schmitz \& Kazyack, 2016). Lilly (2016) highlighted that traditional gender norms and relations are reproduced within such communities; femininity and women are disparaged, masculinity is imagined to be 'in crisis', and feminism is represented as hypocritical and oppressive. Schmitz and Kazyack's (2016) content analysis of the 12 most prominent MRA websites show that there are two primary categories of MRAs with distinctive ideological strategies to provide support for men in pursuit of social legitimacy and power: Cyber Lads in Search of Masculinity and Virtual Victims in Search of Equality. Cyber Lads were shown to utilise themes of explicit aggression towards and the devaluation of women, while Virtual Victims were judged to be more effective, as they adopted political and social movement rhetoric to address men's issues, and concealed

\footnotetext{
${ }^{6} 4$ Chan is an anonymous imageboard website that hosts a wide range of topic forums. It has been identified as an unfiltered breeding ground for racism, misogyny, and extreme content (including images, videos and accounts of interpersonal violence).
} 
antifeminist discourse through the use of legitimated sources (research studies) and issues grounded in truth (men's health inequities) (Schmitz \& Kazyack, 2016).

Several authors have noted that since the emergence of the Web 2.0 era, a particularly toxic version of antifeminism has proliferated across a range of online networks and platforms, in particular the manosphere (Ging, 2017; Gotell \& Duton, 2016; Jane, 2014; Marwick \& Caplan, 2018). Feminist scholarship has brought awareness to online gendered hatred and online misogyny connecting online behaviour to structural sexism and violence against women (Powell \& Henry, 2016). Ging (2017) highlights that the categories and features of the manosphere reflect new toxic assemblages, which complicate the orthodox alignment of power and dominance of hegemonic masculinity within society. The rapid propagation of Red Pill 'philosophy' across multiple platforms and subcultures within the manosphere shows how a compelling cultural motif has succeeded in cross-fertilising and uniting seemingly divergent groups against a common enemy. In an ethnographic study of the social news website Reddit.com, Massanari (2017) reflects on how platform algorithms, design features and community cultures provide fertile ground for antifeminist and misogynistic activism. Using the example of \#GamerGate, Marwick and Caplan (2018) showed that MRAs within the manosphere justified networked harassment by constructing feminism and feminists as villains and, men as their victims. ${ }^{7}$ The term 'misandry', which refers to the hatred of men, was highlighted as a "boundary object" in order to "coordinate and convey meaning amongst ingroup and outgroup participants, depending on the source of its use" (Marwick \& Caplan, 2018, p.11). Such research highlights the (re)production of gendered hatred within online networks such as the manosphere, and how antifeminist ideas proliferate and weave through networks and toxic technocultures (Ging, 2017; 2019).

Recent scholarship analysing the technologically mediated harms of online misogyny and antifeminist rhetoric suggest that misogyny should not be reduced to the isolated antagonisms between individuals or to the outpouring of frustrated trolls alone. Assessing MRA internal discourse, Lumsden's (2019) study analysed MRA discussions of online violence and victimisation on the r/MensRights subreddit. Lumsden's (2019) findings focused on the denial of women and feminists as victims of online violence within these spaces, where MRAs were seen to normalise online abuse and attacks against feminists, arguing that such abuse and

\footnotetext{
${ }^{7}$ The \#gamergate controversy stemmed from a harassment campaign against several women in the video game industry who spoke out on issues of sexism and progressivism in video game culture. For a more in-depth review see: Marwick and Caplan (2018).
} 
trolling was justified as a backlash against men's online victimisation. Lumsden's (2019) research highlights that MRAs police, regulate and control groups/individuals who occupy such online spaces, de-legitimising and 'othering' the victim-status of women or feminists who have experienced online violence and harassment, instead perpetuating an online/offline dichotomy of harms of 'acceptable' or 'legitimate' transgressions based in the terrestrial world (Brown, 2006). The individuals who engage and occupy such spaces are mediated by both the political and local social contexts, including the perceived alienation of certain groups of (white) men (Green, 2019). However, as Green's (2019) work assessing the online communities of the Alt-Right reveals, "these groups are loose and temporary assemblages, rather than coherent and stable communities" which can shift and become flexible where hierarchies of marginalisation become flexible depending on contingent political needs (p. 83). Ging (2017) also reflects:

[t]he loose networks of the manosphere thus materialize and disband around connective conduits of sentiment (Papacharissi, 2010), by mobilizing and reifying narratives of personal suffering to build affective consensus about an allegedly collective, gendered, experience, namely men's position in the social hierarchy due to feminism (p. 16).

Rather, the rhetoric of the declining position of men and the action of networked hate born from such echo-chambers should be understood at the intersection between systemic misogyny and sexism within the wider culture: the racist politics of the Alt-Right, coupled with the technological affordances of various platforms and their attendant (sub)cultures. Such wider structural features (both offline and online) have been shown to augment, amplify, and polarise contemporary politics, maintaining a masculinised and racialised hegemony over such online spaces (Ging, 2017; Ging \& Siapera, 2018; Gotel \& Dutton, 2016; Green, 2019; Lumsden, 2019; Marwick \& Caplan, 2018; Massanari, 2015; Nagle, 2015; Nicholas \& Agius, 2018; Powell et al., 2018). The possibilities for systemic hatred that are further enabled by the internet, combined with a reaction to the perceived diminishing social status of cisgender white men in offline society, has changed the nature and shape of antifeminist discourse in the Web 2.0 era through technosocial processes that are well suited to the amplification of new expressions of aggrieved masculinity (Ging, 2017; Kimmel, 2015). Thus, a critical understanding of such antifeminist technosocial networks within digital society will assist in understanding the 'new' assemblages of extremist masculinity that centre around such tropes of male-victimhood and gendered hate (Powell et al., 2018). 


\section{Incels: hegemony and 'beta-masculinity'}

The majority of explanations of Incels centre around the high-profile mass-killings by Elliot Rodger and Alek Minsassian. Some media and opinion articles theorised that male aggression is caused by a lack of female sexual acquiescence, whilst others centred on the killer's mental health (Hanson, 2018; Louie, 2018). At least one commentator suggested a form of sexual redistribution as an attempt to prevent in an attempt to prevent future mass-shootings (Hanson, 2018). The most commonly forwarded explanations mirrored contemporary academic work on male violence, pointing to 'toxic masculinity' and systemic misogyny as root causes for such unrestrained violence (Tolentino, 2018; Williams, 2018). Psychological explanations for Incel mass-violence centred on symptoms associated with mental health issues such as autism, psychosis, and psychopathy (Allely \& Faccini, 2017). Psychological analyses, however, tend to centre on the individuals of large-scale events, highlighting the barriers and frustrations that the shooters experienced in their day-to-day lives, yet scarcely examine the structural elements involved in such violence (Allely \& Faccini, 2017; Faccini \& Allely, 2016).

Vito, Admire, and Hughes' (2017) thematic analysis of Elliot Rodger's manifesto: My Twisted World: The Story of Elliot Rodger, explored the reproduction of masculinity, sexuality and race present in his mass-killing spree. Rodger constructed his understandings of masculinity in accordance with Western society's hegemonic masculine ideals. Notably, he highlighted the physical embodiment of masculinity, primarily as being White, fit and physically strong (Connell \& Messerschmidt, 2005; Whitehead, 2002), and affirming his masculinity through heterosexual sexual prowess as core facets of being a man (Vito, Admire \& Hughes, 2017). Vito et al's (2017) analysis of Elliot Rodger's manifesto shows that individual-level explanations alone are insufficient. The larger, structural issue of dominant notions of masculine norms, particularly in cases where young men are taught to prove or assert their masculinity through violence, must be better understood in relation to Incels as a wider subculture as opposed to individuals who commit mass-violence.

Incels and other forms of so-called 'beta masculinity' have become a continued topic for debate among journalists and bloggers, whose explanations of "toxic masculinity" mirrored contemporary academic explanations for misogyny and male violence, which ranged from sexual rejection and unstable employment to video-game violence, pornography, and the erosion of white male privilege (Beauchamp, 2019; Tolentino, 2018; Williams, 2018). The term "toxic masculinity" has become widely used in both academic and popular discourses, as 
well as within psychoanalytic contexts, and is used in essentialist terms to describe "the need to aggressively compete and dominate others and encompasses the most problematic proclivities in men" (Kupers, 2005, p. 713). Connell and Messerschmidt (2005), however, argue against such usages as they imply a fixed character type of an assemblage of toxic traits. Hegemonic masculinity constitutes a singular vision of a Euro-centric, heterosexual, middleupper class masculinity that asserts authority over other forms of masculinity as well as experiencing a collective privilege over women (Demetriou, 2001; Kimmel \& Mahler, 2003). Hegemonic masculinity, in some contexts, refers to men's engagement in toxic practices, but since hegemony has numerous configurations, this may include distancing oneself from such toxicity when it is advantageous. Performances of hegemonic masculinity serve to maintain a dominant position for men by subordinating women and non-traditional performances of masculinity, typically through violence, societal structure, and discrimination (Connell \& Messerschmidt, 2005).

Most men are barricaded from achieving hegemonic masculinities' normative standard, yet it is defined as universal and exalted within wider society (Whitehead, 2002). A fundamental ideal of hegemonic masculinity is a presumed entitlement to heterosexual sex, with women perceived as sexual objects or conquests (Connell \& Messerschmidt, 2005; Whitehead, 2002). For men who are unable to have frequent heterosexual sex, such ritualised performances of sexual objectification serve to reduce one's position in the social hierarchy if they are unable to meet this expectation of masculinity (Flood, 2008). Prior academic research on Incels demonstrates extreme responses in failures to achieve perceived normative standards, thus it has been employed as a stable concept used to explain the 'problem' of Incel (Beauchamp, 2019; Ging, 2017; Jaki et al., 2019; Vito, Admire, \& Hughes, 2017). However, Angela Nagle questions the concept of hegemonic masculinity with reagrd to Incels, who appear to openly rail against the normative masculine standard:

But how, exactly, does "hegemonic masculinity" accurately sum up a scene explicitly identifying as beta male? And can "traditional ideas about gender" really be bursting forth from an Internet culture that also features gender-bending pornography, discussions about bisexual curiosity, and a male My Little Pony Fandom? What's more, can a retreat from the traditional authority of the nuclear family into an extended adolescence of videogames, porn, and pranks really be described as patriarchal? (Nagle, 2016, para. 23). 
The 'beta-masculine' style draws intensely from counter-cultural forms, and identifies itself against feminism, but also against social conservatism, political correctness, mainstream consumer culture, and most importantly, against hegemonic masculine norms (Ging, 2017; Nagle, 2016). Nagle (2016) poses a series of challenges in attempting to unpack the 'type' of masculinity displayed by Incels. She questions the accuracy of concepts such as hegemonic masculinity (Connell \& Messerschmidt, 2005) or 'hybrid masculinity,' a position in which Incels self-positioning as victims of feminism and political correctness enables them to strategically distance themselves from hegemonic masculinity, whilst simultaneously compounding existing hierarchies of power and inequality online (Bridges \& Pascoe, 2014; Ging, 2017). Nagle (2016) asserts that such understandings of masculinity do not reflect the amorphous displays of masculinity by Incels.

Criminological examinations of Incels are so-far, non-existent. The violent and misogynist nature of particular Incels at the individual level has received some scholarly attention, however, at the group level Incel research is lacking. Feminist media scholar, Debbie Ging, argued that explanations for violence from within the wider manosphere, including those influenced by Alt-Right ideology, centred on men's claims to victimhood and aggrieved entitlement of the young, often sexually disenfranchised young men that typically inhabit such spaces (Ging, 2017). Blommaert (2017) attempted to understand the online-offline modes of identity and community that influenced Rodger's killing-spree and came to a similar conclusion. The cultural material that emphasised Rodger's perceived victimhood or 'beta' status that proliferated in such "light" online communities provided Rodger with strong ideologically structured templates justifying his resort to extreme destructive revenge on those whom he considered perpetrators of the "crimes" that made him feel such extreme loneliness and unhappiness (Blommaert, 2017, p. 20). In light of the 2018 Toronto Van Attack, previous findings relating to misogyny were further supported by a linguistic analysis of the most prominent Incel online discussion forums, Incels.me. Jaki et al (2019) found that the forum was replete with inciting and enacting explicitly violent fantasies against the out-groups. Jaki et al (2019) however, did suggest that comments might be no more than verbal tactics of selfenhancement within these communities.

Recently, Papadamou, et al. (2020) found that there has been a significant increase in Incelrelated activity on YouTube in comments and videos. This indicates that Incels are increasingly exploiting the platform to spread their antifeminist worldview. The study also indicated overlap with topics relating to the wider manosphere (e.g., the MGTOW movement). Bael, Brace and 
Coan (2019) also found that Incels misogynistic worldview was intimately connected with other manosphere groups and revealed Incels' linkages with several recent acts of politically motivated violence, including Alek Minassian's van attack in Toronto (2018) and Elliot Rodger's school shooting in Isla Vista (2014). The study also highlighted similarities between several 'Incel Saints', such as Marc Lepine's 1989 murder of female students in Montreal, George Sodini's 2009 attack, and Seung-Hui Cho's Virginia Tech mass shooting in 2007. Bael et al., (2019) provides an analysis of how Incel communities support and motivate violent antifeminist action through its use of social categories and causal narratives blaming out-groups for Incel members' sense of pain.

\section{Summary / Academic contribution:}

Empirical research solely examining the 'Incel masculinity' is lacking. Therefore, taking influence from Waling's (2019) critique of contemporary men's studies that typically result in categorical or typological analyses of masculinity/masculinities, this research will expand such analyses of masculinity by not only accounting for the ways Incels negotiate masculinity, but also it will examine the reflexivity of their engagement with masculine practices (Chapter Four). The thesis will also set-out the structural origins of such groups and provide empirical validation to Ging's (2019) assertion that such manosphere cultures can be seen as reflective of the postfeminist, neoliberal society (Chapter Five), as well as the economic anxieties that have been prevalent in Men's Rights circles and what they mean for this specific group of men (Chapter Four). Furthermore, some research has been conducted on how similar cultures radicalise members and encourage extreme opinions, however, apart from Bael et al. (2019), very little has been written on how specifically Incels beliefs and tactics are interconnected with other extremist groups. The 'Black Pill' - Incels uniting ideology - will be thoroughly interrogated (Chapter Six) in order to understand how Incel violence is justified, and weaponised, against women and other out-groups. 


\section{Chapter 3: Methodology and Methods}

\section{Methodological Framework}

The current research sought to accumulate and analyse rich data relating to online misogyny, in order to explore the masculinity of the research subjects, to understand how such cultures foster and weaponize misogyny through new forms of digital technology, and how such online spaces can be seen to foster patriarchal and racist violence within offline society. To achieve this, I adopted a qualitative research design utilizing a critical criminological approach. This chapter outlines the theoretical and epistemological underpinnings for this project, and then discusses how this research was conducted. This chapter also provides a discussion of the relevant ethical considerations, including those specific to digital research methods and techniques. Lastly, this chapter explores the limitations of the research.

\section{Researching Online Misogyny}

This research was borne from an underlying desire to understand and explain a similar kind of misogyny that I - like many young millennial boys living in the digital realm - have taken part in as we navigate these new technological worlds. Many of the posters on Incel forums grapple with feelings of insignificance, invisibility to the opposite sex, and feel alienated and detached from their peers. These feelings mirror some of my early experiences as a young man. Growing up, I tussled with the anxieties of being perceived as 'too fat', 'too ginger', 'not smart enough', 'not good looking' and struggled to get the attention of those who I deemed to be above me on the social hierarchy. Feeling alienated from my peers, in conjunction with being bullied by others, meant that I, too, felt like hiding - and I certainly did. I entrenched myself within the confines of my bedroom playing online video games and browsing online chatrooms (the more graphic and disturbing, the better). I even briefly sought joy and bonded in misogyny, anonymously disrespecting women in online forums such as 4 chan, in videogames and online chat groups. I felt frustrated at the hand I was dealt. Feeling alienated and lost, I uncritically perpetuated many of the discourses and worldviews that the Incels in this study display and fought back against those who I felt so alienated by - including women. I was about 16 at the time, and my life did indeed get better. 
I became aware of the Incel subculture soon after the 2014 massacre perpetrated by Elliot Rodger. After viewing his YouTube videos and his online manifesto, I was particularly interested in understanding how someone who had possibly harboured similar feelings as my younger self could go as far as to murder many innocent victims. In 2018, while completing my early postgraduate studies, yet another mass-murder involving an Incel took place, this time in Toronto, Canada. These events were foregrounded in my thoughts. I saw Incel-related violence as a salient topic to investigate. I also saw an opportunity to get ahead and understand Incels before an attack took place in Aotearoa/New Zealand. This, coupled with the largest terrorist attack on our country on March 15, 2019, implored me to further understand these somewhat similar forms of violence that appear to be happening ever more frequently in Western, democratic nations.

Reflexivity refers to the positionality of the researcher and how that positionality, with its privileges and biases, might affect the research process and analysis. I acknowledge my position as a cis-gendered, Pākehā, man from a working-class background, who has experienced and overcome many of the similar feelings that are espoused by Incel forum users. I also acknowledge my positionality as a pro-feminist academic, and the fundamental opposition that I hold concerning the "Red Pill" and "Black Pill" philosophies.

In this chapter, I outline the epistemological underpinnings for the study and discuss the research design. I then discuss the relevant ethical considerations and reflections of studying online misogyny and vitriol, including those specific to digital research, as well as its limitations. This chapter includes reflexive accounts in an effort to help future researchers navigate potential pitfalls that arise during the research process. It is through this bridge of personal experience and understanding that I seek to unveil a nuanced account of Incels.

\section{Epistemological Underpinnings}

The epistemological concerns of research must be interrogated in order to provide a foundation for the methodology that is selected. Epistemology broadly reflects the way that knowledge and understanding are formed, and it provides the basis for the kinds of knowledge that are possible (Crotty, 1998; Maynard, 1994). There are multiple epistemological perspectives, with positivism on one end of the spectrum and constructivism on the other. Positivist epistemology claims that truth and meaning reside within the entities themselves and views reality as universal, objective, and quantifiable. Positivist approaches have had a long history in 
criminological inquiry and align with quantitative methods, however, such approaches may be considered less appropriate for examining gender constructs which are socially constructed, as well as casting a critical lens on the social institutions that uphold them (Daly \& Chesney-Lind, 1988). The decision to study Incels - a relatively new phenomenon and subculture - dictated an exploratory approach. This meant that I needed to be constantly open and engaged to the possibility of new ideas and understandings of the social world. Given that positivist approaches to research "seek out causal explanations, often phrasing their questions as hypotheses... that set up causal relationships between variables" (Hesse-Biber, 2017, pp. 2122), as well as relying on a more deductive model of logic, an alternative approached was necessary for this study. Due to the exploratory nature of the research, an approach that interprets social phenomena through methods that presuppose that meaning is socially constructed via the interaction between humans or humans and objects was more appropriate (Hesse-Biber, 2017). Such an interpretive approach is in opposition to the positivist paradigm and is line with my own understandings about the nature of reality and what this means for exploratory research.

Standing in contrast to positivist epistemology, the basic contention of constructivism asserts that knowledge is not created through observable phenomena, but rather through individual subjectivity - it is socially constructed by and between those who experience it (Gergen, 1999). It is therefore a consequence of the backdrop in which the action occurs, and it is sculpted by the cultural, historical, political and social norms that operate within that context and time. Furthermore, constructivism embraces the understanding that reality can be different for each of us based on our unique understandings of the world and our experience of it (Berger \& Luckman, 1966). This research is thus framed and informed by a constructivist epistemology. It asserts that the social, cultural and political structures that perpetuate online misogyny are not natural, however embedded they may be, and should not be accepted as fixed; rather, it is essential that they are understood as continuously socially constructed.

In considering the research project's aims and epistemological basis, qualitative methods were employed. The research saw the relationship between theory and research as inductive rather than deductive, in line with qualitative methods that develop theories based on data rather than the positivistic testing of hypotheses against research findings (Hesse-Biber \& Leavy, 2011). This research sought to understand the worldview of Incels within online spaces through the gathering of rich, personal data to enable the analysis of personal narratives, behaviours and 
discourses that are not easily amenable to quantitative analysis (Noaks \& Wincup, 2004). Utilizing a qualitative research design allowed me to collect and analyse multimodal discourses such as verbal language and text, but also provided flexibility to acknowledge the new and creative ways that users interact with technosocial environments. For example, the collection and analysis of comments, 'memes', essays, videos and other cultural material specifically related to, and found on, Incel websites was made possible through qualitative research techniques

Critical criminology has also informed the basis of this research. At its core, critical criminology refers to a group of diverse theories and perspectives that offer a counterpoint to individualist or administrative explanations of crime and social control (Muncie, 2000; Schwendinger \& Schwendinger, 1970). Critical criminologists primarily utilize qualitative and inductive research methods, rejecting the positivistic notions of the neutral observer that are common within much mainstream criminology (Friedrichs, 2009). Critical criminology seeks to identify and challenge power structures and social inequalities that threaten social justice and human rights (Cohen, 1993; Schwendinger \& Schwendinger, 1970). In particular, critical criminology unveils and interrogates the role of state and non-state actors in the reproduction of hegemonic power structures that flourish whilst remaining invisible within the social system. Critical criminology includes a broad definition of crime that focuses on a vast and multifaceted range of harms including sexism, racism, imperialism, economic exploitation and so on, rather than merely the legalistic definitions of crime (Muncie, 2000). Critical criminology, therefore, has the flexibility to be open to understanding new and diverse harms, such as those that are (re)produced within the online sphere.

Orthodox criminology, particularly cyber-criminology, is often preoccupied with developing traditional legal and theoretical frameworks to understand many of the new and evolving crimes and harms within cyberspace (Brown, 2006; Powell et al., 2018; Yar, 2013). Criminology has created a false distinction between 'virtual' and 'embodied' crime, which fails to capture the nature of contemporary society as a "human/technical hybrid" (Brown, 2006, p. 277). The concept of 'digital society' is utilized throughout this thesis to understand the "integrated whole represented by digital technologies and society" (Powell et al. 2018, p. 4). Social life is increasingly mediated through, and interacting with, digital technology (Lupton, 2014). All manner of individuals from offenders, victims, responders to bystanders lead lives routinely shaped by technological and social interaction (Powell et al., 2018). Viewing crime 
through such a frame understands that within contemporary digital society, the nature of social interaction is itself a technosocial process. Powell et al (2018) argue that "[t]echnology is not just revealing pre-existing bigotry within society; rather, technology and society are shaping one another, coalescing in emerging cultures and practices that simultaneously produce and reproduce hate-based harms" (p. 121, emphasis in original). Lumsden and Harmer (2019) implore social science to "throw out the well-worn dichotomies of 'online versus offline', and the 'virtual world' versus 'real world', and instead acknowledge the interconnected and fluid nature of our everyday use of information and communication technologies" (p. 14).

It is from these assumptions that this thesis examines online harms within the Incel subculture. Through understanding digital society in the aforementioned ways, in conjunction with a critical criminological lens which focuses on the ways that institutions and structures reproduce and perpetuate societal harms, this thesis is well placed to critically examine masculinity and misogyny in online cultures. Furthermore, as the Incel, manosphere communities and other forms of 'affective politics' (Ging, 2017; Papacharissi, 2010) increasingly pervade the digital realm, a critical understanding of technosocial networks within contemporary digital society allows for new insights into the increasingly blurred intersections of understanding the world as a human/technical hybrid (Brown, 2006; Powell et al., 2018).

\section{Methodological Approach}

Online misogyny relating to Incels is a relatively unexplored area, so flexibility within the research approach was an indispensable part of understanding the phenomenon. For this reason, a grounded theory approach was considered most appropriate. Grounded theory is a qualitative research methodology that discovers theory through systematic analysis (Strauss \& Corbin, 1997; Glaser \& Strauss, 1967). It involves a systematic process of collecting and analysing data; developing codes to assist with identifying themes and categories; and developing a flexible theory which can be adapted as data collection continues (Glaser \& Strauss, 1967). Adopting a research methodology that was adaptable to changes in data collection methods, as well as analysing the emergence of key themes, was vital due to the fluid and continuously evolving nature of the internet. A grounded theory approach allows researchers to constantly interact with their data, whilst remaining open to emerging analyses and changes (Bryant \& Charmaz, 2007). As the Incel subculture harbours a raft of colloquialisms, idioms and insidejokes, the flexibility of grounded theory assisted in interpreting many of these comments and phrases. 
The study utilised a constructivist grounded theory (henceforth CGT) approach (Charmaz, 2000; 2014). CGT incorporates a specific focus on flexibility and researcher reflexivity, rejects the positivistic assumptions of a neutral observer and understands that all research is bias-laden and influenced by the researcher(s) and the research process. CGT permits the understanding that research is not dispassionate and detached; instead, it encourages researchers to examine their own positionality and how this influenced the research process and the inductive construction of theory (Charmaz, 2000). The reflexive approach offered by CGT also aligns well with a critical criminological approach, as the focus on flexibility and reflexivity allows the data to 'speak for itself', thus aligning with critical criminology's commitment to prioritise the voices of those who are often silenced by hegemonic power structures and norms. It also provides the ability to understand and explain violent and hateful rhetoric in Incel forums, whilst maintaining a critical approach to such discourses.

\section{Research Methods}

\section{Research Design}

This research employed a qualitative approach to investigate online misogyny within Incel forums. The approach incorporated the repeated searching and cross-comparison of online comments on well-known Incel-related forums. Initially, the research sought to utilize Social Media Critical Discourse Analysis (SM-CDA) which is an emerging form of theorization and operationalization that combines principles from Critical Discourse Studies with scholarship in digital media and technology research (KhosraviNik \& Esposito, 2018). Following advice during the research development stage, I decided to simplify the research process and utilize a grounded approach. This still allowed for pseudonymized data to be extracted in the form of comments, 'memes', essays, videos and other cultural material but in a much more streamlined way. This simplification of methods allowed for unrestrained and unfiltered versions of comments and discourses, which were then analysed to uncover significant themes within the data. Utilizing a more straightforward approach to the research shifted focus from the granular analysis of memes and online videos towards a tighter reading of textual discourse found within the online forums. Future study could utilize SM-CDA to reveal how the processes of hyperlinking to other websites and forums aids to proliferate Incel-related discourse throughout the manosphere. 


\section{Data Collection}

I chose the top two threads of highly populated Incel-related websites - www.incels.co (previously incels.me as referred to in other studies) and www.reddit.com/r/Braincels (previously /r/incels) and recorded the data via the screenshot function for each comment thread. ${ }^{8}$ The websites that were accessed were selected due to their large, active and easily accessible communities. Additionally, social-news and community hub, Reddit.com, in particular, has been previously assessed as discussed as being a hub for anti-feminist activity (Massanari, 2017). Incels.co was chosen due to a large numbers of comment threads, an active membership base and extension links to user-generated essays, 'rules', 'FAQs', and archives of Incel-related material, including an 'Incel-wiki.' ${ }^{9}$ Incels.co was also chosen as its predecessor Incels.me was used in previous studies of the Incel community. Additionally, Incels.co was chosen for its status as an independent website, offering its community flexible rules and 'free speech' benefits that may be censored on other forums.

Baele et al, (2019) have highlighted that Incels.co is a quantitatively important location within the broader manosphere, and its predecessor - Incels.me - has been described as "a distinct, sealed-off echo chamber of extreme ideas at the very end of the continuum from "moderate" to radical misogynistic beliefs." (Baele et al., 2019, p. 6). Incels.co was chosen as it is the home to a dedicated Incel community. The Incel worldview has been argued to be present on other forums within the manosphere in diluted form (for example, Sluthate.com was one forum which Elliot Rodger posted his videos and manifesto). Following Baele et al (2019), this study conceptualizes Incels.co as a place of technosocial interactions which seek to radicalize its participants by producing extreme feelings, beliefs and behaviours to encourage violence (against women, other men and at times, themselves).

This research did not directly record or assess demographic information. As the two communities of Incels from Reddit/r/Braincels and Incels.co regularly refer to each other in comments, there is likely some overlap between the groups in terms of its user base. In considering this, unless otherwise stated, the term 'Incels' is used to discuss these two groups

\footnotetext{
${ }^{8}$ Other studies such as Bael et al., (2019) and Jaki et al, (2019) have sourced data from other websites such as www.incels.me and www.reddit.com/r/incels. However, administrators closed down such sites in late 2017 for violating Reddit's community standards, and the incels.me website was shut down by the webhost of the site (Ging, 2017). During the course of the study the administrators of Reddit closed the $\mathrm{r} / \mathrm{braincels}$ forum also for breaking community standards.

${ }^{9} \mathrm{https}: / /$ incels.wiki/w/Incel_Wiki founded July 16th, 2018 and is a user-updatable website that provides various links, information and articles relating to Incels.
} 
of users that post within the the aforementioned forums. As the groups both utilize a pseudonymized account system, it is impossible to say whether the majority of Incels are young White men as much popular commentary on Incels tends to assume (Hanson, 2018; Taub, 2018; Tolentino, 2018). As will be discussed in Chapters Four through Six, this aligns with Incel and the Black Pill ideology which argues that due to women holding power over sexual selection, only cisgender men may hold the label of Incel. Furthermore, due to the anonymity afforded to users by use of pseudonymized profiles, the gender of forum posters cannot be proven. Whilst there are indeed women who identify as involuntary celibate (known colloquially as femcels) in alternate forums, the anonymity afforded in online spaces makes this complicated to determine (see: Appendix 'femcels'). For these reasons, my assumption will be that unless otherwise stated, comments analysed will be those from male-identifying individuals.

Over a period of three months from $20^{\text {th }}$ May 2019 to $20^{\text {th }}$ August 2019 I collected daily content samples of roughly equal size (two blog posts, articles or discussion threads and their comments) from each site. I chose the top commented or pinned thread on each forum, deviating only if the thread was the same as the previous day, which I selected the next appropriate thread. I manually screenshotted and copied the main body of the discussion thread, and created a new folder categorized by the date of capture and then the title of the individual thread. For example, "2307 19 [JFL] JFL at believing the top 20\% meme" highlights the categorization firstly, by date and then the individual thread title. ${ }^{10}$ I then took screenshots of each comment within the larger thread, which varied from one comment to $300+$ comments within a particular thread. To organize and maintain data, I created two master folders, one for the Reddit comment threads and another for the Incels.co comment threads. At the end of the data collection period, I had collected 250 threads containing 10,773 comments.

The collection of such a significant volume of data allowed for the identification of ideological, philosophical and rhetorical tropes and core themes. I also collected additional sources of data which derived from the incels.co forums 'Incel Wiki' page, as well as the 'Rules, Terminology, and FAQs' and 'Blackpill' pages during the analysis phase. ${ }^{11}$ Such pages helped decode the terminology and phrases used by Incels. These and other online sources were utilized to create

\footnotetext{
${ }^{10}$ The term JFL is an acronym for 'Just Fucking Lol' (laugh out loud) and is used to highlight outrageous or comedic content.

${ }^{11}$ The 'Incel Wiki' and 'Blackpill' pages are community-created Wikipedia-like archives of Incel-related information. They are located on the top left hand side of the main homepage of incels.co.
} 
an Incel terminology dictionary (see: Appendix) which holds over 90 terms. For example, early on in the research process I was confused at the phrase "riding the cock carousel." This dictionary also served as a quick-guide for terms I was unsure of during coding and analysis. Such samples were also useful to help me gain insight into how Incels as a group represent their masculinity through discursive practice (Connell \& Messerschmidt, 2005). Data collection was not a static process. After the analytical coding process, I dived deeper into hyperlinked threads within the forums and realized that I required more data to understand the context or background of certain discussions. I overcame this by utilizing the search function of the Incel.co and Reddit forums archives, which allowed me to access historical discussion threads, and in conjunction, more data, which enriched my contextual understandings.

\section{Data Analysis}

I printed and stapled together the data in their relevant threads ordered firstly by date and then by alphabetical order of thread title. During this process, I read the body and comments of each thread and began to write down key themes, narratives and ideas about the topic in field note form. For longer threads, I wrote more detailed reviews of the data and highlighted core passages from the text. Whilst this process was lengthy - and at times, frustrating - it provided me with an opportunity to become more familiar with the dataset. It was also useful as it allowed me to look for common threads and overarching themes within the data, which came to be the building blocks of the analysis. During this stage of the analysis, my goal was to understand the Incel worldview, not from a position of judgement, but one of understanding.

I utilized thematic analysis through grounded theory to analyse the data. Thematic analysis is a data analysis technique for "identifying, analyzing and reporting patterns (themes) within data" (Braun \& Clarke, 2006, p. 79). It involves coding data into systematically developed and refined codes (Braun \& Clarke, 2006; Terry, Hayfield, Clarke, \& Braun, 2017). Thematic analysis is a flexible approach, appropriate for qualitative research, and it is particularly compatible with the constructivist epistemology that underpinned my research. Furthermore, thematic analysis is often utilized within a critical framework, such as that for critical criminology, to interrogate the constructed meanings and significance behind the data (Terry, Hayfield, Clarke, \& Braun, 2017). Thematic analysis processes were applied to the collected content samples. I then manually coded the data using coloured pens and pencils. Once I had completed the initial coding. I checked and refined the codes further and undertook additional coding where appropriate. The process of final codes followed by more specific codes is well 
aligned with thematic analysis that is informed by constructivist grounded theory (Charmaz, 2014).

The themes which emerged during data analysis form the basis of the later analytical chapters of this thesis. Due to the large data set and opting to manually code the data, I selected such themes using rough estimates of the frequency by which a certain code was mentioned. For example, the code 'feminism/feminist' was routinely found in comment threads making it a highly salient theme. As the research is focused on online misogyny, masculinity and understanding how such cultures foster and weaponize misogyny, relevant comments relating to the core themes were salient in almost all threads. As the research utilized a constructivist epistemology, my own individual subjectivity and biases affected the themes and codes selected. This is a strength of the research as by immersing myself within the Incel subculture I am developing an 'insider status' which, overtime, allowed me to understand and react to the subculture through the research process. Through previous experiences with engaging in similar misogynistic forums, my cursory knowledge of the language, cultural references and memes of the manosphere was integral to the research process. However, this positionality may also influence claims to neutrality, as Kanuha (2000) discusses:

For each of the ways that being an insider researcher enhances the depth and breadth of understanding a population that may not be accessible to a nonnative scientist, questions about objectivity, reflexivity, and authenticity of a research project are raised because perhaps one knows too much or is too close to the project and may be too similar to those being studied (p. 444).

However, as Rose (1985) notes (cited in Dwyer \& Buckle, 2009, p. 55) "there is no neutrality. There is only greater or less awareness of one's biases. And if you do not appreciate the force of what you're leaving out, you are not fully in command of what you're doing" (p. 77). I argue that my position as an 'insider' is complicated by at least a decade of learning and personal growth. This temporal distance between a younger version of myself has allowed me to retain the knowledge of such forums whilst being able to critically evaluate the claims of its members and worldviews.

\section{Limitations}

As a process, these data collection methods were useful for categorizing and filing qualitative data. However, the practical limitations of constantly needing to be online and at a computer 
had to be overcome. I developed a system of 'playing catch-up', by which an additional two threads were captured for every day that was missed due to the realities of day-to-day life, work, and other responsibilities. Near the end of data collection, I reflected that there might be an automated way to collect the breadth of comment threads that were generated by the research. Microsoft Power Query, an extension for Microsoft Excel, would be a viable option for those who are engaging in similar research in future to utilize. Microsoft Power Query simplifies data-collection and access by mining the individual comments on a particular thread. It is then uploaded into Microsoft Excel, which would have been then easily exported to Nvivo for categorization and analysis purposes. ${ }^{12}$

A further limitation presented itself in the analysis and writing stages of the research. Due to the expansive data-set and manual sorting, coding and revision it was impractical to search through every coded comment thread by hand. To solve this issue, I created a word document and placed each related series of comments under the heading of particular codes. Comments were added under several applicable codes making this document very large. This meant that it was impossible to utilise the vast majority of quotations or themes in the final thesis, however, selected comments represented the common and core points of the discussion threads they were found within. During the writing phase this was particularly useful as I could easily utilize key word searches to find the relevant data relating to the theme I was discussing.

\section{Ethical Considerations and Research Challenges}

Whilst the internet "has opened up a wide range of new ways to examine human interactions in new contexts, and from a variety of disciplinary and interdisciplinary approaches" (Ess et al., 2002, p. 2), it also brings with it a range of ethical issues for new digital environments which are unique to digital researchers. Researchers of all human research need to be aware of the potential exploitation of participants and ensure that ethical standards are upheld (Noaks \& Wincup, 2004). My task was to evince the problematic behaviours, discourses and worldviews of Incels in an effort to further understand the subculture and their effect on wider societal discourses. My pathway into the content of such culture was not gained through negotiated access or direct participation in its communities. It was accessed through 'lurking' within such

\footnotetext{
${ }^{12}$ NVivo is designed to assist users organise and analyse non-numerical or unstructured data. The software allows for the classification, sorting and arrangement of information; to examine relationships within data; and combine analysis with linking, shaping, searching and modelling. The software also can help the user to make observations and build a body of evidence to support their case or project (Richards \& Richards, 2003). https://digitalcommons.wayne.edu/jmasm/vol2/iss1/4/
} 
publicly available forums in order to access the opportunity to analyse the discourse that the group creates. ${ }^{13}$ My ultimate object of analysis is, therefore, the texts and discourse promulgated within these Incel-identified forums. Though the object of analysis is the texts, discourse and ultimately the worldview of Incels, I had to keep in mind that there are individual people behind the comments. This created several ethical quandaries for digital research.

Firstly, I will briefly discuss issues that may arise from the use of 'grey' data. Rambukkana (2019) refines the term to question whether grey data may be understood as existing in the ethically grey area between analyses of texts (for example, tweets, comment threads, response videos) and human subject research. Are such examples of online data products of human participants? Or are they merely publicly published texts? One of the defining characteristics of internet and digital culture is argued to be the fine line between subjects and texts (Crosset, Tanner, \& Campana, 2018; Rambukkana, 2019). Interactions within digital society are varied, take many forms and can be recorded or captured knowingly or unknowingly between users. Baym (2015, p.7) notes that the "storage" and "replicability" of digital interactions are key concepts for understanding the affordances of communication within digital society. These concepts complicate our understandings of privacy and intimate connection as the line between public and private are increasingly ethereal. Interactions may be public in nature and therefore textual (tweets, blog posts, comment threads, YouTube videos). However, authors of such content may neither expect nor welcome researcher attention to their online interactions or even understand that such research is possible (Basset \& O'Riordan, 2002; Rambukkana, 2019). Therefore, what are the ethical responsibilities of the researcher within such dubious 'grey' areas when deciding to pursue research?

The Association of Internet Researchers (AoIR) set of guidelines asserts that:

...ethical decision-making interweaves one's fundamental worldview (ontology, epistemology, values etc.), one's academic and political environment (purposes), one's defining disciplinary assumptions, and one's methodological stances. Decision-making occurs at many junctures in the cycle of inquiry, including research design, research conduct, and research production and dissemination (Markham \& Buchanan, 2012, p. $3)$.

\footnotetext{
${ }^{13}$ The term 'lurking' refers to silently and invisibly watching people within an online space.
} 
It has long been acknowledged that ethical decisions involve the researcher's worldview, the political environment and that wholly objective research is an unattainable ideal (Haraway, 1988). Rambukkanna (2019) asserts that in politically charged cases, such as researching the "alt-right", despite there being grey areas in ethical decision-making, researchers hold a responsibility to speak truth to power and interrogate, deconstruct and expose the influence of potentially harmful groups on the wider society in the pursuit of social justice.

There are also risks inherent in researching some online groups, as some groups can be hostile to research. For example, Bergstrom's (2016) study involving the online videogame World of Warcraft discussion forums highlighted that the gaming community had begun to experience survey fatigue, with many researchers attempting to solicit research participants from the forums. Bergstrom (2016) also found that research was met with heavy criticism and mistrust from the community, and which resulted in verbal insults to the researcher and research process. Other problems arise when attempting to research other bastions of male-dominated internet culture, such as the backlash received by feminist writers and researchers reporting on the \#GamerGate ${ }^{14}$ controversy (Marwick \& Caplan, 2018; Massanari, 2017). Rambukkanna (2019) suggests that given such backlash to being researched, a resolution to the grey area problem between subjects and authors may be found in treating digital materials as published texts. This was a strategy that was useful in Pollock's (2009) study to help protect against backlash onto the researchers and their institution. The study used covert, invisible nonparticipatory research to study White supremacists' use of newsgroups. Pollock (2009) highlighted that members were aware and demonstrated their knowledge of posting in a public forum through their in-group discussions of the likelihood of being observed by authorities online. Pollock (2009) used a strategy of anonymising the conversational data, even though the open public nature of the data allowed the research to proceed without informed consent. Aside from the protection of the subjects of study, Rambukkanna (2019) importantly notes that the anonymization also was done to protect and isolate the researchers - and, by extension, their institutions - from a potentially toxic, dangerous or legally risky proximity to their subjects.

Another risk inherent in the study of hostile groups, in particular, is that those who participate in wider online harassment and other abhorrent behaviours (i.e. some groups within the manosphere) invoke additional challenges. Marwick and Caplan (2018) highlight the

\footnotetext{
${ }^{14}$ The \#gamergate controversy stemmed from a harassment campaign against several women in the video game industry who spoke out on issues of sexism and progressivism in video game culture. For a more in-depth review see: Marwick and Caplan (2018).
} 
networked harassment that has occurred when feminists and female public figures expose female structural inequality and violence. The harassment experienced by feminist scholars is networked, in the sense that it is coordinated and organised through a shared vocabulary, worldview and hyperlinking of articles, blogs and other material that is spread across the manosphere (Marwick \& Caplan, 2018). Powell et al (2018) highlight this to be a feature of digital society, adding that the nature of online hate and bigotry is a technosocial process which results from ongoing cycles of technological and social interaction. As such, a risk in studying hostile groups such as those within the manosphere is that there may be blow-back on researchers, their family and institution (Rambukkanna, 2019).

Incels are extremely self-aware of their public appearance. In the dataset, there are many references to outsiders of the forum 'lurking' and screenshotting comments within non-Incel forums. Incels are highly aware of the way that society perceives them and that they are indeed watched and observed. In the current study, one user compared themselves to "animals in a cage" with the public watching their every move. Posts on the Incel.co and r/Braincels forums critically reflected the recent BBC documentary Inside The Secret World of Incels (2019) and routinely comment on news about white male terrorism, rape and domestic abuse of women, and paedophilia. Posters are also particularly critical of media outlets and the public at large when discussing Incels. Located within the 'Incel Wiki' page on Incels.co there is a repository of academic studies and resources related to Inceldom, and several threads debate the validity of academic - namely feminist - arguments against Incels. They also highlight academic studies that provide support to their worldviews. Such threads are referred to as "LifeFuel" and include one notable thread which is titled "Academics defending Incels", which provides links to YouTube commentary and academic studies of 'Incel-Allies'. This shows that users of the Reddit and Incel.co forums are aware that they are being observed, and therefore should reasonably expect that comments posted on such public forums might be used for research purposes.

Feminist research into online misogyny (such as Marwick \& Caplan, 2018; Ging, 2017, Jane, 2016) also highlights risks for researchers, their friends and their families. As such, I made the decision not to name friends and family in the acknowledgements section in this thesis to prevent potential backlash against my loved ones. During the preparation for this research, I also heightened the security settings of my social media and public websites where possible to prevent potential backlash. This decision was not made lightly, and due to fears of doxxing, 
stalking, threats and other forms of online violence that feminist media commentators experienced during the \#Gamergate controversy, coupled with the aggressive antifeminist rhetoric discussed within Incel forums, following Rambukkana's (2019) advice for researching potentially aggressive online groups it was decided appropriate to restrict the publishing of personal information within the thesis.

The Victoria University of Wellington Human Ethics guidelines state an ethics application is necessary under the conditions of 13.1 (a) "whether the persons who posted the data would be realistically aware of its availability to third parties" and due to the nature of publicly available and searchable material within the online sphere, this project was determined to not require an ethics application. Further, (d) of the same article discusses anonymity regarding the data of the research project. In both forums, all users require the use of a pseudonym, which, following Pollock (2009), I have omitted in the final analysis to protect the anonymity of forum users. The descriptions and quotations of comment threads are referred to "a user on Incels.co" or "a user on Reddit" or similar terms to completely remove any association with particular users.

Sensitive social science research of this nature has been assessed to have, and in my experience certainly has had, negative mental health consequences (see: Rager, 2005; Sanders, Munford, Liebenberg, \& Henaghan, 2014). Throughout the research process, I kept a diary to reflect on, and try to minimize, the harms that are inherent in such sensitive research. This helped me to rationalize what I was viewing in the online forums. It also allowed me to express myself and vent about how this research emotionally affected me day-to-day. In reading the reflexive journal back and viewing my entries, it was clear that the daily drudge of examining hostile and extreme misogyny took its toll on me. For example, there were times in the early datacollection phase of the research where I started to think like an Incel. I began to judge people, particularly couples on their 'level' within the sexual marketplace (as will be discussed further in Chapter Six), and for a short while became paranoid about my daily interactions with people: Were people to be trusted? Did they judge me solely on my appearance? Did I meet the supposed standards of hegemonic masculinity? Are the claims Incels are making about politics valid, if so, have I just taken the 'blue pill' and am I living a life of delusion? These feelings and questions came to dominate my thoughts, and exacerbated feelings of social anxiety. This made me adjust the data-collection method to avoid viewing and analysing the comments as I collected them. I also began making sure I strictly recorded my thoughts in my journal. This helped me express my feelings and helped me to reframe what I was seeing, to bring me back 
into the 'real world' and to 'take me out' of the research at the end of the workday. I also regularly debriefed with academic colleagues throughout the research process. Dickson-Swift, James, Kippen, and Liamputtong, (2001) implore researchers to be mindful of how the research process affects them in the face of "ongoing challenges such as dealing with developing attachments, hearing untold stories, feelings of guilt, vulnerability and exhaustion" (p.344). Such emotional issues are fundamental to being reflexive of the research process and findings (Berger, 2015).

Finally, it should be noted that to further protect the anonymity of the Incel.co and r/Braincel members I have opted to exclude references to names, dates and the forums associated with the data. In the remaining chapters, all italicised and unattributed quotations represent the data collected and analysed from the two Incel forums chosen for this study. Additionally, definitions for obscure linguistic terms and phrases are located in the appendix section of the thesis if they are not immediately footnoted. 


\section{Chapter 4 - Masculinity and Incels}

The world creates the monsters and then acts like it had nothing to do with creating them. People constantly have to hide behind a façade that they are a good person. It's fucking beyond pathetic and disgusting. Words cannot express how fucking enraging this shit is that humans do.

In order to understand the subculture of Incels, it is vital to interrogate the structural contexts of their antifeminist perspectives and virulent misogyny. Using rich thematic data, this chapter begins by sketching a caricaturised version of Incels' conceptions of hegemonic masculinity colloquially known as "Chad". I then discuss Incels' self-positioning in relation to Chad, to deconstruct their version of 'beta-masculinity'. The chapter provides a brief commentary on the effects of economic instabilities on young people caused by shifts to modern-day neoliberal capitalism, outlining how such an environment has affected working class men in particular. I argue that the same shifting economic environment has also negatively affected men like Incels, who are also characterised by uncertain employment opportunities. I argue that these economic anxieties further reinforces and reproduces a 'crisis of masculinity', through which Incels virulent misogyny can be viewed as an attempt to reassert and reproduce an atavistic patriarchal ordering of society through the subordination of women, alongside other nontraditional performances of masculinity via online misogyny and, occasionally, extreme offline mass-violence.

\section{Ideals of Hegemonic Masculinity: Painting the picture of 'Chad Thundercock'}

Incels believe that the world is organised through group categorisations within a structure of a three-tier social hierarchy based exclusively on physical appearance (Bael et al., 2019). At the top of this hierarchy is a minority of physically attractive 'Chads' and 'Stacys'. ${ }^{15}$ Below them lie the majority of average-looking 'normies'. At the bottom of said social hierarchy lies a minority of physically unpleasant 'Incel' individuals (exclusively males), who are victims of involuntary celibacy. This hierarchy is directly aligned to the social ordering of the 'Black Pill', which understands the world as biologically determined and ordered through a racialized patriarchal hierarchy. The Black Pill concept will be discussed in greater depth in Chapter 6.

\footnotetext{
15 "Chads" refers to the anti-Incel, a man who is sexually successful, charismatic, and handsome and fulfils the expectations of the dominant mode of masculinity.

"Stacy" refers to the idealised embodiment of femininity as described by Incels. A sexually attractive, popular woman.
} 
Incels position themselves discursively and hierarchically in relation to 'Chad', referencing a 4Chan meme devoted to 'Chad Thundercock', a caricaturised version of the dominant notion of masculinity. Nagle (2016, para. 27) explains that "Chad is a stand-in for the young, attractive, muscular football player claiming dominance over the beta-world in the contest for sexual success with women." Theoretically, 'Chad' is a label primarily ascribed to male bodies who perform or possess a handful of attributes or traits that are persistent of the hegemonic masculine standard. Such standards include aggression, toughness, hardness, ableness and competitiveness (Whitehead, 2002). Scholars have suggested that the physical embodiment of hegemonic masculinity depends on the dominant characteristics of size, stature and conventional (White) male attractiveness (Light \& Kirk, 2000; Ricciardelli, Clow, \& White, 2010). Incels commonly portray Chad as a 'Sports Jock' archetype, which is usually represented as White, popular, charismatic, socially, sexually and economically successful, often unintelligent and competitive. Competitiveness, particularly within the realm of sports achievement, is a defining characteristic of the archetype of the hegemonic masculine identity (Kupers, 2005; Bird, 1996).

Importantly, Chad also embodies such physical, emotional and economic components of hegemonic masculinity, but these are less important than the paramount marker of masculinity, his ability to have frequent, heterosexual sex. Masculinity scholars agree that a presumed entitlement to women as sexual objects is a core facet of hegemonic masculinity (Connell \& Messerschmidt, 2005; Harrington, 2002; Kimmel, 2008; 2014; Whitehead, 2002). Incels conceptualise women to be agentless in their desire to have sex with Chad. Instead, they are presented as both sexual objects and conquests for Chad. Incels conceptions of Chad signal a full transition to manhood, as heterosexual sex is understood as a requirement in the transition to becoming an adult male (Carpenter, 2005). Men who fail to have regular heterosexual sex is a signal to others of either sexual incompetency or virginity, which serves to raise suspicions of homosexuality (Carpenter, 2005; Flood, 2008). Incels conceptions of Chad portray him as a hypersexualised, alpha male that frequently has heterosexual sex (sometimes even without paying as in the context of sex work for example):

Let's assume that Chad is having sex 1 hour a night for at least a couple of nights a week. In a year, this equates to approximately 100 hours of sex (although realistically they get much more). Assuming a prostitute charges $\$ 150$ for an hour (realistically a decent one is much more expensive), an Incel would need to PAY \$15,000 a year to get what Chad gets FOR FREE! Chad is able to pay less and thus able to earn less, which 
means he is able to WORK LESS in order to obtain the same prize as an Incel, because he was born with good genetics (something out of his control). Because an Incel was born with inferior genetics (something also out of his control), he will have to compensate for this by working much harder to get what Chad gets JUST FOR EXISTING.

The construction of Chad conforms to representations of the archetype of westernised characterisation of hegemonic masculinity (Connell \& Messerschmidt, 2005). Hegemonic masculinities have cultural particularities (Harrington, 2002), for example, Incels note various cross-cultural conceptions of different 'Chads'. These reflect such socio-cultural constructions of hegemonic masculinity. For example, African American 'alpha males' are referred to as 'Tyrone', while 'Chang' refers to an East Asian conception of hegemonic masculinity. Incels also commonly refer to 'Chadpreet' as the Indian archetype of hegemonic masculinity. These conceptions of the male body are not biologically driven, but rather a socio-cultural and historical construction that reflects culturally defined ideal characteristics of the dominant group (Harrington, 2002; Whitehead, 2002).

Note the inherent racism in such cross-cultural representations of 'Chad', particularly due to Incels conceptions of the Western (White) construction of Chad is the most common and dominant expression of the characteristics that are deemed desirable. This conforms to Connell and Messerschmidt's (2005) conceptions of hegemonic masculinity where 'whiteness' is valorised and accepted as the hegemonic standard. Green's (2019) discussion regarding 'extreme right' communities also reflects this idea, noting that within such forums, heteronormative white males are placed as intellectually, morally, and racially superior, while other ethnicities are argued to occupy a subordinate position within a hierarchy of inferiority.

Cross-cultural constructions of Chad - particularly African American conceptions - are imbued with a focus on hyper-masculinity, and an emphasis that such a masculinity is violent and dangerous. Such animalistic and overtly racist conceptions of Black and Brown men are congruent with stereotypes of the dark-skinned criminal that are rooted in, and perpetuated by, media stereotypes (Levchak, 2018). Such tropes are used by Incels to exaggerate stereotypes of Black and Brown men and reinforce racial hegemony. Grieg (2019) refers to such narratives in relation to Far-Right communities as the construction of "barbarous masculinities" (p. 77). Such narratives centre on the predatory male other to mobilise fear and strengthen ethnonationalism. For Incels, Black and Brown 'Chads' are associated with violence and sexual 
threat. These supposed threats from the barbarous masculinity of the dark-skinned Chad are particularly salient, when considered in the context of the hetero-patriarchal caste system to which Incels subscribe. They therefore represent a significant threat to the construction of Incels' masculinity.

However, the threat of violence or economic challenges are reduced when referring to 'Asian Chads' (Chang) due to the supposedly effeminate nature of Asian men. The wider focus on the heteronormative, Western (White) ideal of masculine virtues may then be understood both as a measuring stick by which Incels position themselves against, as well as a form of protection against the perceived existential threat of the 'barbarous masculinity' of outsiders. Such presumptions about the 'threatening' masculinities noted by Incel groups are connected to the radicalising discourse of the Black Pill, as well as mirroring narratives espoused by the FarRight (Grieg, 2019; Green, 2019).

Incels thus position themselves against the embodied characteristics of their conception of the masculine-ideal. At once, these characteristics reflect the fears and anxieties of Far-Right communities in their conceptions of the barbarous masculine other, while also embodying Incels own wounded sense of masculinity (Grieg, 2019). This embodiment depends on the selfsurveillance of one's own body to conform to such dominant discourses of masculinity as well as policing by others (Foucault, 1978). Failing to achieve such an idealised standard, Whitehead (2002) reflects that "their sense of masculinity is invested in such attempts" (p.191), and the ideal continues to be reinforced within Incels' online communities. However, to be a 'Chad', it is not just necessary to fulfil the physical archetypes of hegemonic masculinity as one user suggested that "To be an ultimate Chad, I think it can't be a matter of looks and attractiveness alone. You have to embody the idea that you can get away with being a complete piece of shit, and have no remorse for it." To be a Chad, it is also important to negotiate the world with a certain irreverence.

In a discussion of who is "the ultimate Chad" archetype, various film and sports stars were suggested, focusing on social, sexual, career and economic success as markers of being a 'Chad'. One user suggests that:

Leonardo Dicaprio is the ultimate Chad. He existencemogs all of us combined. He's tall, smart, attractive, good at acting and shit (THE ULTIMATE CHAD OCCUPATION), gets paid to kiss hot women, bangs female celebrity after female 
celebrity, he's funny, extremely rich... We are useless compared to him. He can do EVERYTHING a thousand times better than we can.

This suggestion was the most highly supported within the thread. Many comments endorsed this conception by reposting the comment with the phrase "High IQ" - a common way of expressing agreement. However, this challenges the 'Sports Jock' archetype discussed earlier.

To be a Chad, it is also important to dominate over men who are lower than they are within the social hierarchy, whom Incels" refer to as "low status men" or "beta-males". The term 'existencemog' used in the previous quotation means to overshadow, to block out into obscurity. Being a Chad implies greatness and a sense of universal superiority over others. Incels consider 'Chad' to be the ultimate (yet undeserving) victor in the genetic lottery, and often argue that everything good in life comes to him easily. Many Incels have a tenuous relationship with 'Chad'. Simultaneously, Incels revere Chad, with comments admiring elements of his 'alpha' personality:

Love is for Chad only. Chad gets hand-fed grapes by his harem, while a slave fans him with a giant fan. Chad cannot cry (not that he ever needs to, as his life is literally perfect). Chad cannot have feelings. He must be stoic and never show weakness. He is the ultimate alpha-male.

Despite such reverence, Incels also express a powerful disdain for Chad, and position themselves on the moral high ground against the mythologised version of the archetype of the dominant performance of masculinity:

The glorification of Chad's and Stacy's is so illogical and weird. Chad's are not capable of feeling love because they are degenerates who have lost the ability to pair bond. These unintelligent man-beasts are out there pumping and dumping hot thots on a regular basis, but what are they actually contributing to society. Women flock to these idiots and reinforce their aggressive and sometimes violent pursuit of pussy.

Research has shown that a failure to maintain the socially ascribed traits of hegemonic masculinity can have deleterious effects on men, such as negative body image, depression, and eating disorders (Barlett, Vowels, \& Saucier, 2008). More recently, Scaptura (2019) and 
Scaptura and Boyle (2019) have demonstrated that men who do not uphold such 'Chad-like' representations of masculinity are less likely to feel accepted as men and are, in turn, more likely to show aggression towards outsiders such as women and minorities. Incels failure to conform to such masculine expectations result in them describing themselves as 'beta-males'. Consigned to such apparently biologically determined positions within society, Incels often refer to the maxim of "alpha fuxx, beta buxx" which describes how women sexually desire alpha males but rely on beta-male providers for emotional and financial support. Incels consider Chad to be the ultimate winner in all aspects of life, representing a caricaturised version of popular cultural representations of hegemonic masculinity.

The complexity of the 'Chad' - Incel relationship is two-fold. At once, Incels revere Chad for being a confident, sex-having alpha-male, while simultaneously loathe him for achieving the societal expectations of what it is to be 'a man'. By self-positioning themselves as 'betas' and protesting hegemonic masculine norms, Incels self-conceptions of their own masculinity appear diametrically opposed to the hegemonic ideals of masculinity from a surface level appraisal. However, as the next section will demonstrate, the 'beta masculine' style that identifies itself against feminism, political correctness and the hegemonic masculine norms of 'Chad', also subordinates women and other non-traditional performances of masculinity through virulent online misogyny and hatred (and, occasionally expressions of extreme offline violence).

Revealing the Incel (beta) Masculinity:

A growing body of research has traced the evolution of reactionary antifeminist movements (Banet-Weiser \& Miltner, 2016; Ging, 2017; 2019; Jones, Trott, \& Wright, 2019; Marwick \& Caplan, 2018; Schmitz \& Kazyak, 2016), and these largely consist of 'angry, straight, white men' (Coston \& Kimmel, 2013, p. 380). Some MRA groups argue for the rejection of hegemonic masculinity, while others champion and valorise it (Kimmel, 2013). Over time, coinciding with feminist concerns gaining ground (albeit at a glacial pace), the feelings of MRA communities have turned to that of rage, fear and anger, with the main aim being the (re)appropriation of power. Feeling that women have stripped such power from men, Kimmel (2013) argues that men's anger rises from the culmination of two sentiments: 'aggrieved entitlement' and a sense of victimisation. Aggrieved entitlement refers to a state where men believe themselves to be entitled to such power, through a combination of historical position, biological destiny, so-called moral legitimacy, and when it is not received, feelings of 
victimisation occurs (Kimmel, 2013). Much of this sense of entitlement and victimisation stems from men who are part of contemporary online MRA movements. Groups of 'angry white men' such as Incels and other interconnected online manosphere cultures primarily centre on 'geek' or 'nerd' forms of masculinity. These groups have been shown as atypical of the archetype of hegemonic masculinity, however, they have engaged in varying degrees of hostile misogyny as a reaction to an increasingly equalising society (Banet-Weiser \& Miltner, 2016; Ging, 2017; 2019).

Nagle (2016) problematizes understanding the masculinity of Incels as hegemonic, highlighting that the style of manosphere cultures such as Incels "draws from a countercultural genealogy" which importantly identifies itself against the hegemonic masculinity of 'Chad'. Nagle contends that such explanations fail to acknowledge the non-hegemonic 'beta' culture of the internet and asks, "can a retreat from the traditional authority of the nuclear family into an extended adolescence of videogames, porn, and pranks really be described as patriarchal?" (2016, para. 23). Ging (2017) argues that such men's performances of masculinity should be described as 'hybrid masculinities', as their self-positioning as victims of feminist misandry and political correctness allows them to strategically distance themselves from hegemonic masculinity, while simultaneously compounding existing hierarchies of power and inequality within online spaces. Bridges and Pascoe (2014) note that such 'hybrid masculinities' involve the selective incorporation of elements of identity that are typically associated with marginalised or subordinated masculinities, while also utilising elements of privileged men's gender performances and identities to reinforce existing inequalities when it suits them.

I argue that Incels self-positioning as victims of feminist misandry and evolutionary biology allows them to utilise a hybrid model of masculinity which in turn allows them to valorise and respect 'Chad' (their conception of dominant masculinity) while marginalising and attacking those lower on the gendered and racialized hierarchy, and other subordinated or marginalised masculinities (cucks, normies etc). ${ }^{16}$ Incels create an enemy of feminism, women, political correctness and 'soy-boys' in order to channel their sense of aggrieved entitlement to conventional markers of masculine 'success' (which they are unable to access). ${ }^{17}$ In doing so, Incels perpetuate 'traditional' or hegemonic ideas of masculinity. Nagle (2016) rejects the

\footnotetext{
${ }^{16}$ 'Cuck' refers to a man whose wife/girlfriend forces him to watch her have sex with another man (usually a black man). For an in-depth genealogy of phrase see: Lokke, 2019.

'Normie' refers to anyone who is not an Incel, Chad or a Stacy.

${ }^{17}$ 'Soy-boy' refers to studies that suggest that soy lowers testosterone. It is often used to refer to progressive men as an insult directed at their masculinity.
} 
notion that 'beta' masculinity is congruent with typical models of hegemonic masculinity, however, it effectively does same thing: it reasserts a patriarchal social ordering of society through a hostile form of online misogyny. Incels utilised the 'scientific' Black Pill to argue for a reordering of society that necessarily reduces women's rights. The reasoning for such alienated and marginalised individuals' preferences of a patriarchal society are reflected here:

What happened is that the foids changed, Jew feminism infected the world, and it's continuing to infect the world. Jew feminism allowed the foids to be free. ${ }^{18}$ In actuality, foids should be treated as property of men, to be distributed as men see fit. Ideally too, foids would be bred to increase the female:male ratio in order to create a positive environment for men. We would be using the latest biotechnology for the benefit of MANkind. However, thanks to Jew feminism, we are using technology to supress men.

Incels' sense of 'aggrieved entitlement' and victimisation occurs in relation to what they view as a sexually and socially unequal society brought on by the social progress of the women's liberation and feminism (Bael et al, 2019; Ging, 2017). Such feelings of victimisation and aggrieved entitlement are a core facet of the Incel identity. By externalising blame for their lack of sexual and social success on feminism, these men restore dominance against those who caused their humiliation (Kimmel, 2013). Like Elliot Rodger, many of these men feel culturally marginalised as they cannot live up to the dominant conceptions of masculinity, and misdirect their feelings of anger externally towards women and men with less social status instead of those who occupy the positions of power within society (Vito, Admire \& Hughes, 2017). Incels belief in, and regular assertion of, the notion that "feminism is the root of all evil" derives from their own real-world experiences that have left them bereft of sexual power. Women's right to refuse sex has left such men feeling powerless in situations where they believe that they are entitled to it (Anderson, 2005; Kimmel, 2008).

Such narratives concerning the 'wounded masculinities' of Incels parallel Grieg's (2019) conception of Far-Right masculinities. These tropes originated in the men's rights movements of the 1990s that proclaimed men to be in crisis at the hands of feminism (Messner, 2016). These narratives reflected the cultural and economic dislocations experienced within the neoliberal globalised era, and how such societal changes inflict gender-specific injuries to (White) men. In Far-Right and Incel narratives, these broad socio-cultural changes are represented as an existential crisis for both men and society (Grieg, 2019; Green, 2019).

18 "Foid" or "Femoid" are short for "female humanoid" and is used to 'other' or dehumanise women. 
Narratives combine and reflect threats to power and hegemony. The barbarous other's masculinity represents the fear of taking employment opportunities and bringing a wave of sexual and drug-related violence. The loss of power to women through feminism represents the fear of men's supposedly diminishing status within society. Kimmel (2003) highlighted that White supremacists understand that such messages of loss and displacement feed on undercurrents of discontent and build fertile ground for radicalisation. In order to attempt to reassert power, Incels have committed mass-violence to repair their fractured sense of masculinity. However, these attempts to reassert male dominance within society most often derive through varying intensities of misogynist comments. For example, comments often discuss calls to reassert patriarchy:

Incidentally, women USED TO be shamed for their bad behaviours, but feminism made it acceptable - sometimes even illegal - to shame women for almost anything. When women are empowered and allowed to freely choose their sexual partners, beta males will compete with each other in order to pander to them. But if women are the property of their fathers and husbands and don't have any rights, then men don't have any rational reason to pander to them.

Incels often claim that political correctness, liberal culture and feminism does nothing for "lowstatus" men like themselves, even going as far as claiming that 'beta-males' are oppressed by progressive politics: "I predict this becoming more common, not just with height, but other factors like facial aesthetics, hair loss, etc. Increasing hypergamy is weighing on men and influencing how we are being treated. An increasing amount of men have nothing to lose."19 Such themes of perceived oppression are further explicated in the following comment, which blames feminist discourses for the subjugation of 'low-status' men such as themselves. Here, expectations of the physical embodiment of hegemonic masculinity fuse with the sense of aggrieved entitlement and victimisation brought on by an externalisation of blame towards women and feminism.

\footnotetext{
${ }^{19}$ Hypergamy - A term borrowed from biology used to described the phenomenon of females mating with males of a higher status. MRAs and Incels apply it to humans, arguing that women attempt to find men who are higher status than they are. This gives birth to what they call the 80:20 rule: that the top 20\% of men are being competed for by the top $80 \%$ of women, and the bottom $80 \%$ of men are competing for the bottom $20 \%$ of women.
} 
Yeah and they'll blame "toxic masculinity" for it. They'll be pro mental health one day, bully a short guy the next day, then film this the following day and just call him a bitter Incel with a terrible personality. The circus is currently happening. Honk honk!

Highly visible representations of masculinity such as height and strength are regularly referred to and compared within Incel forums in relation to 'Chad'. These representations are both often revered and disparaged. It is clear that Incels cast blame on women and feminism when they are directly threatened by the physically constraining social and cultural standards of hegemonic masculinity.

Such an obsession with physical attractiveness can also be seen in the numerous discussion threads and polls titled 'rate my face' or 'who here has the worst genes?' which are usually followed by comments debating who has the best physical justification for being an Incel. For Incels, "your life is in your looks", and for those without preferable genetics they are purportedly consigned to a miserable, unsuccessful life. Numerous threads discuss the importance that men must be over 6-foot-tall in order to be sexually successful. This is particularly common in threads discussing the online dating market, where one user suggests that "If you thought women were picky in real life, wait until you try Tinder. Many of them will actually specify a 'minimum height' of at least 6 feet." One user references an article that discussed the top 10 keywords for dating app success where it was found that men who mentioned that they were above 6 feet tall were more successful. One user suggests: "Men want love, women want 6" because roasties are inhumane creatures incapable of love and only view men as a status symbol to enhance her own social position. ${ }^{20}$ Here, another user urges others to subscribe to the belief that height is the most important factor when it comes to attractiveness and sexual selection, thus the most important prerequisite of manliness in the eyes of an Incel:

The only reason being 6-foot-plus is so coveted by women is because women don't view men as unique human beings who should be valued for their inner qualities or individuality. They're simply status symbols and adornments for women. In a vacuum, a women would not have her physiological or psychological arousal magically turned off by looking at a really attractive guy who happens to be one or two inches below some arbitrary measurement... women see it as an area of competition with one another. Before she can see how good his face, body, or personality is, you need to get

\footnotetext{
${ }^{20}$ 'Roastie' refers to a woman who has had sex with more than one partner. It is used to simultaneously body and slut shame women.
} 
his height, because it more easily gives a number that can represent his "value." Just as a woman can flaunt her 6-carat ring over her friend's 5-carat ring, so too can she flaunt 6-foot-something over 5-foot-something. This preference is born of two things and two things only: arbitrary measurement systems and the innate superficiality of women. It is by no means natural or unchangeable."

Women's 'sexual selection' is a frequent and important theme that highlights a reversal of the central tenet of hegemonic masculinity which emphasises the sexual domination of men over women (Connell \& Messerschmidt, 2005; Kimmel, 2008; 2014; Whitehead, 2002). Aside from the lack of agency that women have in relation to sex with 'Chad', when deciding whether to have sex with men who do not represent the epitome of the hegemonic masculine ideal, women are concieved as the primary agent for 'sexual selection'. This enhances feelings of powerlessness and threatens Incels sense of already wounded masculinity.

For many of the Incels within the current study, their involuntary celibacy appeared to be a stigmatised status that challenged their masculine identities. This finding was congruent with Vito et al's (2017) analysis of Elliot Rodger's manifesto, finding that Rodger's status as a virgin was a source of significant tension as he cited being a "kissless virgin" and a "lonely virgin" throughout his manifesto. References to "kissless virgins" are common and highlight frustration and anguish with other people's perceived sexual success. For example, one user describes, "I'm a sperg, ADHD, everyone at my school thinks I'm mentally ill, I get told I look like an alien, and I am a kissless virgin. Sometimes I feel like I'm not a man. While I'm still trying to escape inceldom, posting here is one of my copes." For this user, his sense of masculinity is directly associated with, and therefore challenged by, a lack of sexual attention from women. Additionally, his identity and involvement in the group norms of the Incel subculture are directly related to 'coping' with his state of involuntary celibacy. Engaging and being involved in the Incel community is consistently referred to as a positive way of dealing with the user's sexual and social alienation.

In addition to the deep desire to engage in sexual relationships with women, Incels also frequently reaffirm the notion that women owe men sex, and that failure to provide this pleasure is in direct conflict to a man's sense of masculinity (Connell \& Messerschmidt, 2005; Anderson, 2005; Kimmel, 2008). This was also found in Rodger's manifesto, with the shooter reflecting that he deserved sex and romantic relationships (Vito et al., 2017). Commentators have argued that a form of sexual redistribution should be made available for Incels and other 
mass-shooters to prevent future mass-shootings (Hanson, 2018). Incels have also echoed these arguments in relation to sex-work stating, "It is so frustrating. Whores should be sent by the government to alleviate our sexual frustration. Because, we are handicapped of getting that hot coochie." Such narratives obscure personal responsibility for mass-violence and misogynist behaviour and reinforce notions of masculine entitlement to heterosexual sex (Connell \& Messerschmidt, 2005). Casting blame externally to feminism and women, Incels appear to reinforce each-other's feelings of sexual malaise and alienation within the forums, allowing them to 'cope' with their Inceldom.

However, these understandings of Incels primarily desiring sex are put into tension by one commenter who argues, "just think of the feeling of having a woman wanting you. You are important, your life has value." Here, the Incel user suggests that feeling valued and validated by a woman is central to Inceldom, rather than merely having sex. Another user in a different thread echoed this sentiment:

The sex act itself is not the point. Go see an escort if you want, you will still be an incel. You will still be treated like shit at best and dangerous at worst by society. You will still never know what it is like to be respected as a human. Inceldom is not about sex.

This finding challenges the orthodoxy of op-ed and non-academic, popular readings of Incels, which argue that Incels obsession with sex, if fulfilled, may reduce the likelihood of users engaging in violence. These conclusions centre on typical conceptions of Incels that usually highlight the slew of misogynist and hateful discourse, as well as the occasional brutal massmurder events that are associated with the movement. However, Incels do actively discuss the unrealistic expectations of masculinity that many Incels hold and argue that the unrealistic hypersexuality of the caricature of 'Chad' act as attempts to confirm masculinity, and how these understandings actually work to harm men who hold such conceptions of masculinity.

And there are people here so fucking delusional that they think most males are getting sex [frequently and consistently] and that Chad's harems are a meme. Males are programmed to act like they are getting laid to be shown as successful; they aren 't even getting sex in marriage.

Such fantasies of Chad and the unrealistic expectations on other men's sexual success may serve as a form of protection against others compensatory masculinity. As Connell (1992) has 
shown, the construction of dominant forms of masculinity does not occur in isolation, but in relation to other forms of masculinity and femininity. When one's masculine identity is challenged by their subordinate position in relation to others, they may engage in compensatory masculinity (Pyke, 1996). The constant disparaging of women and 'lesser' men as a way to reconstruct their 'true' masculinity and compensate for their beta-masculinity in comparison to Chad also highlighted this. This finding is congruent with Jones et al's (2019) research into the Men Going Their Own Way (MGTOW) forum, which harbours a more benign and passive form of misogyny in comparison with Incels. Jones et al (2019) found that groups like MGTOW try to reclaim and reinforce hegemonic masculinity in response to the perceived loss of power they experience in mainstream society, compensating through intense misogyny and sexism. However, such compensation was not always prevalent, which complicates these understandings. As the previous quotes suggest, some Incel users consider feeling valued and validated by a woman as central to Inceldom, rather than merely having sex.

The final, and most telling, example shows Incels hyper-awareness of their own and other's relegated positions as "low-status" men in contemporary society. Analysing a picture of an Incel who has been noted as trying to flirt with women online, the user acknowledges the 'crisis of masculinity' men, and is portrayed as representative of men who inhabit the wider manosphere:

I think it's just sad. Men are clearly broken. Sometimes I hate these cucks, sometimes Ifeel pity for them... so many of them live lives of misery and quiet desperation. I mean, just look at that guy, the glasses, the haircut, the bland background (is that some basement he's sitting in? At least that's no wallpaper) ... probably poor, overworked but underpaid, maybe living with his parents again, no sex, no romance, somehow looking both older and younger than he is at the same time. And longing for some romance, intimacy, sex, sensuality, flirting with the other sex he goes online ... and never having learned anything other, he doesn't know what else to do than giving cringy compliments. A short back and forth, then no one replies anymore. The weather, the internet, I'm not like all the other guys... that's it. And at 3 AM he goes to sleep again. Or to bed. At 5 AM, he finally finds some rest...

This user paints a caricature of the idea of the typical 'beta-male', the archetype of the Incel. These findings suggest that Inceldom is also about a broader sense of alienation and a wider 'crisis of masculinity'. Incels certainly place importance on an identity cast in relation to the 
hegemonically masculine ideal, as well as a fundamental opposition to feminism and political correctness, but their wounded sense of masculinity is also centred on a larger 'crisis of masculinity', and a perception of victimisation and aggrieved entitlement (Kimmel, 2013; Grieg, 2019). It is also clear from the previous section that Incels hold the competing notions of wanting to be valued by women, whilst hating and disparaging them. Such a paradox views women as both a source of would-be meaning, love and as a source of happiness, whilst simultaneously one of fear, anger, and extreme hatred. These contradictions also extend throughout other narratives central to the Black Pill, and will be revealed throughout the remaining chapters. The next section examines a final, and important, theme relating to the conception of Incels 'beta masculinity', which is that of waged work.

\section{Economic Anxiety and the 'beta' masculine style}

Waged work occupies a central and defining place in the construction of masculinity (Connell, 1999). For men (especially), work has offered a key route from the transition from adolescence into adulthood. The employment options that would likely facilitate and secure such a transition between 'boy' and 'man', have been increasingly fractured due to the deindustrialization of large swathes of the industrial labour force in Western neoliberal countries. As such, the terrain on which men construct their masculine identities has shifted profoundly over the recent decades. The decline in industrial employment and trades, the rise of the service industry and the relative labour market success of young women appears to make this negotiation from adolescence to adulthood all the more uncertain. For many working-class youths in advanced industrial economies, a linear transition from school into employment is increasingly difficult, and cycling in and out of short-term precarious work and making little progress into more stable career options is increasingly commonplace (Bourgois 1995; France 2008; Furlong 2006; McDowell 2003; 2019; Nixon 2017). For those with poor educational achievement, work may be hard to get at all or confined to occupations such as those in the service sector, where 'feminine' qualities of deference and docility are emphasized alongside demands for interpersonal skills (Kenway \& Kraack, 2004; McDowell, 2003; 2019; Salzinger, 2016).

For men and the construction of masculinity, Kimmel (2016) explains that such cultural and economic changes are important as "[t]he downwardly mobile lower middle class has more than just its economic position at stake; the class is defined by its economic autonomy" (pg. 22). Amongst them are small business owners, craft workers, highly skilled (formerly) union protected manufacturing workers, and small-scale farmers. All these livelihoods have a 
commonality; they are hangovers from an industrial society centered on the male breadwinner role and are based on 'traditional' forms of masculinity. Economic instability and shifting work practices have disproportionately affected young working-class men (Kenway \& Kraack, 2004; McDowell, 2003). Like the Far-Right masculinities in Grieg's (2019) study, Incels report such economic dissatisfaction and blame women, 'leftists', and feminism for their declining economic positions.

It's already happening. They have excluded us from the job market (automation is coming, they don't need us anymore), the dating market, our freedom of speech is being limited at each day and women worship is increasing like crazy (see that gamer gurl whore selling bathwater). It's over, you can see in the statistics, the number of young men killing themselves is increasing and will increase more with the passing years because there's no way of ascension anymore. They've taken so much of us and they are still not satisfied. We will have a rebellion or the number of men killing themselves will get bigger and they won't give a fuck. I bet in the second option.

This reference to 'ascension' has a dual meaning: on the one hand, it represents Incels failure as men to enter into a sexual relationship (a key facet of adult masculinity), whilst on the other, for many young men it refers to the increasing difficulty to acquire stable waged work. Other threads go further, with one user highlighting the turbulent and inflated economic conditions facing youth: "the job market is tough for young people. All the companies want entry level applicants to have a tone of experience by don't want to pay them adequately." This is explained alongside the collapse of the traditional family unit: "the family system has disintegrated because of feminism... Family stability has been replaced with a quest for sexual hedonism... Not only is marriage not honored socially, but it's very morally corrupt with law enforcement and justice system heavily favouring women over men." The user also argues that these issues cascade to create "the collapse of the community" arguing, "socially, we've become increasingly atomized... Two things that have contributed to the disintegration of the community is multiculturalism and individualism." This user argues that because of feminism and multiculturalism this has caused a blurring of a shared set of values: "[w]ithout adherence to a common set of shared values there can be no support system. And without a support system we have things like homelessness, addiction, and depression." Another user in the thread agrees with the original poster, going on to explain: 
The common link between these issues is surely feminism, although it is cause and effect at the same time. It's not the only factor, but I would say the most important of them all. The ongoing speculative bubble on pussy creates an avalanche of new bubbles, such as the one on housing. You have double the people competing for the same quantity of houses, or even less houses because people are discouraged to build new ones due to the leftist and tax hungry economy. Femoid (((independence))) is always a very costly mistake. The ridiculously long list of requirements is not necessary for most of the jobs out there, unless, just my opinion here, it's because they have to balance the incompetent femibeasts they hire with overqualified and underpaid people.

In the same thread, many comments reinforce the notion that waged work is a prerequisite for the construction of masculinity (Connell, 1999). "Incels are screwed in more ways than sexual exchange. In fact, most young men are screwed financially and socially whether Incel or not. To become a man, you must enter the workforce which is increasingly hard to do nowadays." In the post-industrial working environment, White masculinity is increasingly pathologized and problematized as feminist and multiculturalist politics gain steam (Odin Shaw, 2019). This is a particularly 'White' and Far-Right reaction to such a problem. Far-Right leaders mythologize the discourses of the ethnic majority as a nation-family under siege from both internal and external threats - particularly from people of colour (Galasso, 2019). In reality, through the austerity politics of neoliberalism, Black men (as welll as people of colour and women) have suffered economic injuries, precarious work and unemployment at higher rates than Whites in the U.S. In addition, Black communities continue to experience a host of harms such as environmental racism (for example, the Flint, Michigan water crisis see: Pulido, 2016), greater economic strain, higher rates of incarceration and over-policing due to 'tough on crime' laws, as well as longer periods of unemployment than White men (Benach, Amable, Muntaner, \& Benavides, 2002; Hunter \& Robinson, 2016). Such narratives that construct White masculinity as under threat from feminism and multiculturalism are deployed to engage support for the reassertion of a White, ethno-patriarchy by the alt-right, and as Galasso (2019) highlights, these narratives have also proliferated amongst other grops throughout the manosphere.

McDowell (2019) demonstrates how young working-class men are particularly disadvantaged in competition for employment in the interactive service sector. Many threads allude to Incels self-characterisation as 'NEET' (Not in Education, Employment or Training) and living in their 
parent's homes. For Incels who have yet to enter the adult world of waged work or higher education, the service sector roles, which stress 'feminine' characteristics of deference, cleanliness and a pleasing manner, are difficult to come by. Galasso (2019) reflects that "by reifying the nation-family as the traditional family, the Far-Right aims to normalize xenophobia, ethno-nationalism, and authoritarianism" (p. 105). It must be emphasized that Incel communities are not only made up of White men. However, Incels disaffected and wounded sense of masculinity, coupled with their likely disadvantages in competition for employment (McDowell, 2019), creates the foundation from which radicalization can occur through such appeals to the 'nation-family' style rhetoric of the Far-Right (Zimmerman, Ryan \& Duriesmith, 2019).

However, as Nagle (2016) has highlighted, "In the information age, the tastes and values of geeks are elevated above the masculine virtues of physical strength and material productivity that proceeded them." (p. 40). Many Incels who do manage to go on to university appear to flock to employment within the IT and STEM sectors. This was identified as a way to navigate their social and sexual alienation, and thus their masculinity - to 'wealthmaxx'. Wealthmaxxing is seen as an alternative site to display power and masculinity. For one user "Hoping to go back to uni so I can moneymaxxx and not die of a lack of healthcare in this American shithole country. If I can get enough money, some foid will at least want me to be her betabuxx provider.” One particularly prolific Incel.co user suggests:

If I can wealthmax enough, I will go and Currymaxx, take advantage of their culture and exploit it to their benefit. I'd go around to all of the poor villages and communities looking for a diamond in the rough, a cute young virgin who's dirty and her clothes are in tatters. I'll approach her father and offer him a chance to save his daughter from a life of poverty and some money to help maintain his household, and more likely than not he'll accept the offer. Easier than trying to obtain a feminazi beast with ideas of 'empowerment' here in the West.

The attempted reassertion of patriarchal modes of organization that is inherent throughout Incel discourse can be seen within such a context as a backlash against the shifting economic and cultural concerns that are touted by Far-Right narratives to not only disadvantage blue-collar men, but also masculinity in general (Grieg, 2019). A focus on traditional masculinity is a coreradicalizing theme for the Far-Right, which has been demonstrated to be congruent with the men's rights politics of the wider manosphere (Ging, 2019; Grieg, 2019; Green, 2019; Odin 
Shaw, 2019). Such a fear of female (and racial) encroachment on 'men's work' is clearly highlighted as a threat to Incels sense of masculinity. Banet-Weiser and Wiltner (2016) contend that "the injury of economic recession is transformed into male injury, specifically caused by women" (p. 173). Incel rhetoric directly reflects this, adding that the core features of multiculturalism and feminism have interacted to encroach both on the online and offline social spaces for men, but also in the workplace. Women and minorities are seen as taking jobs (including those in the STEM and technology fields) that are the "natural" right of men. Negra and Tasker (2014) suggest that such economic crises circulate and create zero-sum thinking amongst men's rights circles, which works to demasculinize men as women move further into the workforce.

\section{Summary}

It is apparent that Incels sense of victimisation is most often blamed on women - their perceived oppressor. This fundamental opposition to women's 'natural' instincts of sexual selection are used as an explanatory mechanism to understand their lack of social and sexual success. Feeling marginalised for failing to live up to the dominant conceptions of masculinity, they direct their feelings of anger externally, blaming women for being "picky" and highlighting the supposed "innate superficiality of women" to explain their sense of alienation and powerlessness (Anderson, 2005; Kimmel, 2008). These concerns are not new, as they parallel pro-feminist concerns of the early Men's Liberation movement, which underscored that the 'male sex role' was destructive and even lethal for men's health, emotional lives and relationships (Messner, 2018). However, as several scholars have highlighted since the early 2000 's, the advent of the Web 2.0 saw a more widespread antifeminist men's discourse promulgated throughout the manosphere (Ging, 2017; Gotell \& Duton, 2018; Lilly, 2016; Marwick \& Caplan; 2018; Schmitz \& Kazyyack, 2016). These groups continue to focus on framing men as victims of a 'politically correct', feminist-inspired moral panic (Gottel \& Dutton, 2018). This continued 'masculinity in crisis' narrative has expanded with the perception that women, LGBTQ people, and people of colour are gaining too much power in social, sexual and economic realms at the expense of male power (Ging, 2019; Grieg, 2019). For these 'aggrieved' men, rather than acknowledging the neoliberal economic roots of their changing circumstances, blame is cast onto women, ethnic minorities, 'social justice warriors' and identity politics in general (Ging, 2019). 


\section{Chapter 5 - 'Postfeminism' and Incel backlash}

So far, in order to explain the virulent misogyny of Incels' I have interrogated the structural context of hegemonic and 'beta' masculinities within the enduring economic anxieties and instabilities of the neoliberal era. As per the calls for masculinities scholars to examine how the social and cultural context of postfeminism (O'Neill, 2015) impacts on masculine subjectivities and men's practices, I examine such claims in regards to Incels virulent misogyny and antipathy towards women and 'PC' politics within such an environment. In the first section, I provide a brief overview of postfeminism and explicate how postfeminist culture has allowed the space for such toxic online men's politics to proliferate through the depoliticisation of feminism as a movement and its individualising narratives of gender politics (as per Ging, 2019). In the second section I evaluate Incels' relationship to feminism as per Ana Jordan's (2016) Feminism, Postfeminism and Backlash Typology, using rich thematic data to illustrate Incels' relationship to postfeminism, arguing that such neoliberalisation of gender politics has resulted in a backlash against perceived feminist ('PC' culture) gains. Finally, the chapter closes with a discussion which frames the contemporary men's rights politics of Incels as underpinned by intensely personal concerns, rooted in narratives of sexual rejection and pseudo-scientific explanations of the sexual marketplace, which are as (Ging, 2019) argued reactionary features of the postfeminist cultural environment.

\section{Postfeminism as antifeminism?}

The rapidly changing and contradictory media constructions of gender of the late 1990s and early 2000s, which simultaneously celebrated 'girl power' and female success while casting hostile scrutiny of women in the public eye caused some gender scholars to be sceptical of the 'post'-feminist optimism that was seen to be emerging (Gill, 2017; Jordan, 2016; Negra \& Tasker, 2007). As an academic and media concept, postfeminism is often understood as an epistemological shift in feminist thought towards a period where feminist goals of equality have been achieved. Likewise, this notion of the 'pastness' of feminism is reflected within popular media and culture, particularly following the period of second-wave feminism (O’Neill, 2015; Tasker \& Negra, 2007). Eschewing the temporal notions of postfeminism, Gill (2017) explains, "postfeminism is as much a neoliberal sensibility as one defined by its relationship to feminism. It may be best thought of as a distinctive kind of gendered neoliberalism” (p. 611). As Ging (2019) highlights, unlike second-wave feminism, 
postfeminism is not a form of political activism, but rather a fluid and contradictory set of responses to the perceived successes and failures of feminism.

The term postfeminism has been understood as a "backlash" against feminism (Faludi, 1992; Gill, 2007). Faludi (1992) notes that there has always been resistance to women's rights, which subsides and resurfaces periodically. The points at which resistance to women's rights become acute are defined as backlashes. Jordan (2016) highlights that "[b]acklashes do not spring from the void, they feed on undercurrents of antipathy towards feminism and are symptomatic of its (perceived) successes. Backlash is, by definition, parasitic on feminism and only becomes necessary when feminism is strong, rather than declining” (p. 22). However, several feminist scholars highlight that while the concept of backlash is useful in understanding the social and cultural forces that work to counteract and undermine feminist progress, feminism has an enduring history of being met with strategies of resistance, negotiation and containment (O’Neill, 2015; Tasker \& Negra, 2007). Rosalind Gill (2007, p. 254) also notes that the backlash thesis fails to take stock of the changing misogynist discourse:

Much sexism, it seems to me, operates without the alibi of nostalgia for a time when men were men and women were women, but is distinctively new. It has to be understood not only as a backlash, a reaction against feminism, but also as a new discursive phenomenon that is closely related to neoliberalism.

McRobbie (2009) and others (Gill, 2017; O’Neill, 2015; Tasker \& Negra, 2007) posited that postfeminism, and by extension postfeminist culture, is defined by antifeminist sentiments which suggests that gender and sexual equality have been achieved. Postfeminism's repackaging of female sexual objectification, self-enhancement and consumerism as empowering to the individual silences the vocabularies for discussing both structural inequalities and cultural influence. Kelen (2009) suggests that, instead, postfeminism intensifies the surveillance and expectations on individual women's bodies. Patriarchy is thus reterritorialized within the individual, through the logics of freedom of choice and selfdetermination, masking structural examinations of power. Gill (2017, p. 609) suggests that the 'ordinariness and everydayness' of postfeminism is problematic due to it being

a sense-making characterized by relentless individualism, one that exculpates the institutions of patriarchal capitalism and blames women for their disadvantaged positions, that renders the intense surveillance of women's bodies normal or even desirable, that calls forth endless work on the self and that centres notions of 
empowerment and choice while enrolling women in ever more intense regimes of 'the perfect'.

Postfeminism has also moved gender politics from the realm of the political to that of the cultural, which has allowed for a host of music, television and literary icons to occupy key symbolic spaces in the discourse of gender politics (Gill, 2017; Ging, 2019; Tasker \& Negra, 2007). This has significantly expanded the visibility of feminism, thus leaving it open to attack (Ging, 2019). Postfeminist media discourses routinely locate feminism as anachronistic since it portrays gender equality as being (more or less) achieved thus is now a consumer identity or lifestyle choice (Jordan, 2016). McRobbie (2009) argues that postfeminism is "a new kind of antifeminist sentiment" where "elements of feminism... have been absolutely incorporated into political and institutional life," such feminism has been co-opted and watered down for the broader neoliberal project (p. 7). Postfeminist culture is mutually synergistic with neoliberal economics as well as bio-essentialist understandings of gender difference, rolling back decades of gendered, social constructivist research (Gill, 2007; Ging, 2019). Neoliberalism's ascendancy maintains the gender order as the postfeminist neoliberal subject ostensibly has no gender. This allows for the silencing of women's interests and the maintenance of men's dominant structural positions. Further, Ging (2019) highlights how "[postfeminism] locate[s] a plethora of social and economic problems - and their solutions - in the bodies and minds of individual women" (p. 51). The 'ordinariness and everydayness' of postfeminism and neoliberalism celebrates the rapid 'de-gendering' of the workplace and society, arguing that women must merely 'lean in' more to become successful rather than working to combat systemic inequalities that disadvantage women (Gill, 2017; Ging, 2019).

It is within such a postfeminist cultural context, and a renewed luminosity of feminism, that feminism finds itself is also prone to profoundly misogynist attacks (Gill, 2017). Banet-Weiser and Miltner (2016) reflected that if feminism is popular, then so too is its antithesis - virulent misogyny. The rise in popular, institutionalised, and professionalised feminism (Messner, 2016) co-exists, albeit uncomfortably, with antifeminism (Ging, 2019; Gill, 2017). Like contemporary manifestations of the feminist movement, men's rights activists have also shifted their gender politics from the political to the cultural realm, focusing less on collective action and more on the individual (Ging, 2017; 2019). The technical affordances of social media within the Web 2.0 era have allowed an expansive array of cultural production of memes, inside jokes and other affectively charged tools to communicate gender relations (Ging, 2019; Papacharissi, 2014). Each MRA space has its own memes and cultural icons, but all are united 
in Ging's (2019) understanding that "memes function less as calls to political action and more as channels for the collective venting of anger.” (p. 54). In this way, complex gendered debates are boiled down to easy tag lines or phrases such as "feminism is the root of all evil" or claims such as "white women are oppressors" that simplify and express narratives centred around personal victimisation.

Such collective venting of anger that Ging (2019) alludes, at least in the case of Incels, appears to centre on a defining paradox: structurally, men hold institutional power in patriarchal society, but most men do not feel very powerful. The remainder of this chapter is therefore framed around the idea that despite living in a society where men hold privileges and advantages over women, much of Incels anger is a backlash towards feminism due to a perception that they do not hold such power. The individualist understandings of gender dynamics that are privileged within a postfeminist neoliberal society provide a foundation for backlash against feminism and the perception that women are trying to gain an unfair advantage over men. Incels do not feel as though they are powerful, thus any feminist gains exacerbate the sentiments of anger, resulting in vile online misogyny and sometimes, leading to extraordinarily violent events.

The next section applies Ana Jordan's (2016) Feminism, Postfeminism, and Backlash Typology to the case study of Incels. Labelling Incels as a 'movement', and thus analysing their specific discourse, is challenging due to lack of structure or leadership, and their affinity for 'trolling' (Nagle, 2016). As previously discussed, Incels' make up a small, yet vocal section of the wider manosphere. Ging (2017) has highlighted that it is no longer appropriate to refer to them as groups but rather as loose networks or assemblages, which "materialize and disband around connective conduits of sentiment" (p. 653). United by a virulent hatred of feminism, the nebulous and shapeshifting networks of the manosphere cross-pollinate 'philosophies' and narratives of each group. The narratives and philosophies of Incels' straddle other forms of the five key categories of online men's politics that Ging (2017) has previously revealed. Incels narratives and cultural tropes centre chiefly on women (and feminism) being gatekeepers of sexual selection (for men who do not possess the traits of hegemonic masculinity as seen in the discussions of 'Chad' in Chapter 4). Most of the discussions relate to the dark, nihilistic philosophy known as the 'Black Pill' (which will be expanded upon in Chapter 6) which explains social life through the lens of a carefully selected positivist worldview. The intention for the remainder of this chapter is to evaluate such narratives in relation to Jordan's (2016) typology to evaluate some of the key antifeminist narratives present in the data. 
Located within the 'new' strand of MRAs that have been shown to be motivated by highly personal accounts and individual concerns and united by an antipathy towards feminist politics (Ging, 2017), it was unsurprising to find little evidence of support for feminist concerns within the data. Mirroring Jordan's (2016) evaluation of RF4J, ${ }^{21}$ there was no suggestion that Incels, or men generally, could find allies in feminist organisations. This finding was congruent with Jones et al's (2019) study on the MGTOW group. For men who did engage with feminist discourses, or who were seen to be actively fighting for progressive concerns were routinely ridiculed and labelled as "white knights", "cucks", and "social justice warriors." 22 Alongside the labels attached to such men who claim alliance with feminist or other 'progressive' causes, was regular mocking in comment threads. For example, 'Incel Tears' (IT) is an Incel 'watchdog' subreddit that is committed to revealing many of the harmful discourses that are propagated in Incel forums. Male IT members are constantly referred to as effeminate 'social justice warriors' and are actively disparaged within r/Braincels and Incels.co.

Male IT members are particularly derided for being 'soy-boys' and 'cucks'; thus, presented as not holding the prerequisite virtues to keep their sexual partners satisfied. After disparaging a particular IT member during a back and forth dispute, one Incel user suggests, "[he] must have been mad about his bull drinking all his soylent after ravaging his wifey all night long." Here the Incel user implies that due to being allied to a perceived feminist cause, the IT user has bound to be 'cucked' by his partner with a more aggressively masculine sexual partner, claiming dominance over his wife as well as his home (by finishing his soy-milk beverage). Lokke (2019) highlighted that the term 'cuck' as deriving during the 2014 \#Gamergate controversy, and demonstrated the relationship between cuckold porn and cuck memes depictions of white masculinist anxieties. The masculinist and racist framing of this term is utilised to police the IT user by framing his engagement with feminist discourse as indicative of a supposed failure to satisfy his sexual partner, and uphold his sense of masculinity (Lokke, 2019).

\footnotetext{
${ }^{21}$ RF4J refers to the UK fathers' rights group "Real Fathers 4 Justice" (Jordan, 2016)

${ }^{22}$ White Knight refers to a man who takes things out of context to adamantly defend women's rights. 'Cuck' or 'Cuckhold' refers to a man whose wife/girlfriend is having sex with another man, usually a more dominant or aggressive alpha male.

Social Justice Warrior refers to a person who expresses or promotes socially progressive views (viewed in a negative frame).
} 
Incel users actively police commenters that display feminist or 'progressive' sensibilities. As Green (2019) has highlighted in his study of the Reddit and 4chan communities of the AltRight, the policing of 'insiders' works by 'othering' political dissenters which threaten the intellectual, moral and racial superiority of straight White men. Incel members often abuse those who, at the very least, do not show antipathy toward feminist or politically correct concerns through performative expressions of ridicule. Users will often accuse other Incel users who express politically progressive sentiments as members of the out-group. "Women's hypergamy and sexual selection knows no bounds. Women are free and equal to do whatever they wish in today's society, so they choose Chad. STFU IT cuck faggot." In this highly supported comment, this Incel user reveals their antifeminist position and cast dissenters as outsiders by utilising hostile aggression to shut down what they may perceive as pro-feminist and progressive points of view. Such attacks also serve as a warning against future dissenters who may threaten the hegemony of the White masculine superiority within such online spaces (Green, 2019).

Such narratives align more to backlash narratives, however, in the dataset Incels did sometimes admit to gender power imbalances. When they do discuss the concept of 'power', it is almost exclusively split between two overarching narratives. First, White men are perceived to be disadvantaged not only because of feminism, but also because of progressive gains generally. This narrative is more closely aligned to Jordan's (2016) backlash typology, which I discuss later in this section. Second, Incels discussions of power also centre on the notion that women are the sexual gatekeepers in heteronormative relationships:

Women have the power for mating in today's society. It's sexual selection. It's literally based in biology. It's seen in many species. Bigger male species tend to be selected for and have a harem of females. While smaller "beta" males get zero pussy.

Here, it is evident that this user believes that women hold an immensely disproportionate power of choice over whom she wants to have sex. This explicitly masculinist narrative centres on the notion that all men are always 'up for it' (with sexually desirable women), therefore, women hold the power to decide on sexual matters (as discussed in Chapter 4). For Incels, as a sexually and socially alienated group, such understandings are explained in reference to the Black Pill (Chapter 6), which Beauchamp (2019) describes as "a profoundly sexist ideology... that amounts to a fundamental rejection of women's sexual emancipation, labelling women as shallow, cruel creatures who will choose only the most attractive men if given the chance." 
Here, Incels utilise selected biological and evolutionary psychological perspectives to explain women's lack of interest in them. In highlighting women's sexual agency, Incels acknowledge that gender imbalances exist within society. However, such claims were often made to downplay women's inequality in other areas.

To police such antifeminist narratives, Incels attempted to emasculate other users who show dissent by critiquing Incels 'Black Pill' philosophy. There was some acknowledgement of a gender imbalance regarding women's sexual gatekeeping. This finding suggests that a simplistic evaluation of gendered power dynamics was present within Incels narratives. However, feminist ideas were scant throughout the data and tended to discuss a postfeminist cultural environment ambivalently, discussing 'feminist' and 'progressive' issues with little analysis of power dynamics, or collective struggle, aside from when such imbalances were perceived to affect men. For example, Incels were constantly presented as victims of women's oppression and highlighted men's subjugated status within contemporary society.

Postfeminist sentiments were more common within the Incel discourse than discussions acknowledging feminism (in non-backlash terms). Postfeminist individualist narratives centred less on gender-neutral, formal equality/rights claims as seen in Jordan's (2016) analysis, and and were focused more on 'proving' that feminism was no longer necessary due to women's supposed equality. For example, in one thread where users debated whether women whether women were happier under a patriarchy, the top comment suggests, "Women get to ride the cock carousel and still have a beta provider waiting for them at the end of it all." 23 This narrative suggests the belief in a gender-neutral world where patriarchy has been dismantled; implicitly arguing that feminism is no longer necessary. This finding was also illuminated through frequent calls that "feminism is the root of all evil". However, Incels believed that this was undesirable and unnatural, a refrain that is also echoed within Far-Right communities (Greig, 2019). The following comment describes a common narrative concerning women's perceived power over men in society:

Women these days have near unlimited power and choice so of course they're unhappy. They pretty much run the world while men slave to please them. The only reason they're unhappy is because they're burning out on the hedonic treadmill, they have literally so much choice and so much provided to them they seek more and more to stay satisfied

\footnotetext{
${ }^{23}$ Cock carousal refers to what Incels think that women do before eventually settling down. The idea that women have sex with as many "high quality" men as possible, moving from one to another without though. Women are typically between the ages of 16-25 during this so-called phase while they are at their 'peak' SMV.
} 
but never satiated. So yeah, they're depressed, but mostly as a result of greed than anything else.

Such rhetoric frames women as irrational and unable to make well-informed choices for themselves in a (so-called) post-patriarchal society. This line of thinking is consistently repeated, and women are framed as child-like, or animalistic with vague references to academic literature and 'data', which are seen to provide legitimacy to such claims. Ging (2019) notes that the individualism of such a postfeminist culture is utilised within the manosphere's conceptions of gender differences:

Postfeminist culture is also deeply enmeshed in mutually synergistic alliances with neoliberal economics (Gill, 2007; Gill and Scharff, 2013) and bio-essentialist accounts of gender difference... Both of these systems are appealing on the basis of their reliance on individualism; because they eschew systemic or structural analysis, they locate a plethora of social and economic problems - and their solutions - in the bodies and minds of individual women (p. 151).

Incels use of biologically-determined explanations for human behaviour are used to cast assumptions that all women act in accordance to their nature. Building on comments that present data and graphs suggesting that women are happier under overtly patriarchal conditions, another user asserts that:

We know from the data and science what makes women happy, and its patriarchy, women may think they don't want it, but they do. They're very child-like and primitive when it comes to decision-making, it's like when a child who doesn't want to take a bath because they're not forced to. A child will be happier when their body isn't rotting, and they aren't sick when they are forced to take care of themselves, but I'm sure there are many kids who don't want to.

The comments within these threads utilise biologically determinist and evolutionary psychological concepts and data to argue that gender equality has been achieved, thus feminism is no longer necessary in such a gender equal society. In utilising bio-essentialist understandings of gender difference, the use of a postfeminist frame that also argues women are the gatekeepers of sexual selection, Incels in effect conceal the wider gendered inequalities that pervade society under the guise of their selective interpretation of science. 
Routine narratives that infantilise women and reduce the category of 'women' to essentialised biological animals further complicate an analysis of postfeminist discourse. Many narratives discuss violence against women and blame the violence on the victimised (women). These portrayals attempt to de-gender women and evade structural analysis of wider violence against women and girls (VAWG) by naturalising male violence in readings of 'scientific' understandings of VAWG, thus questioning women's 'decision' to date (obviously violent) men. For example, in the thread titled, "Nigger writes book about domestic violence, gets killed by boyfriend" users comment on an article where a domestic violence researcher was murdered by her ex-partner (Oppenheim, 2019). The top comment suggests, "Bitch was so in love with Chad that even tho she was against domestic violence she still stayed with him even tho he beat her and had to call the police to her house 19 times!" This narrative is common within the remainder of the thread; instead of blaming the perpetrator of the violence, blame was cast on the victim and what she was not doing to protect herself.

Incels narratives evaded structural examinations of gendered violence, instead actively blaming the victim and blaming 'PC culture' generally for misrepresenting intimate partner violence (IPV). This user shifted the conversation to minimising men's role in such violence and suggested that women should take responsibility for their own safety:

Domestic violence has become a topic heavily virtue signalled by those seeking to further one's career or status in the PC world. The issue with all of these Domestic Violence advocates is that they NEVER put ANY blame on the woman; they NEVER discuss how or why these women have selected these men. The women has 0 responsibilities for anything in regards to domestic violence, because the man hit the women. It is $100 \%$ the mans fault, there is no in-depth understanding of how the violence originates.

Women are held responsible for the success of intimate and family relationships, and men are judged under expected norms of relational dominance and control; both are held responsible for fixing their abusive situations (Stamp \& Sabourin, 1995). However, a consistent refrain from Incels (and other MRA groups) is that women (feminists) take the side of women in matters of VAWG and are involved in a conspiracy to subjugate men (Dragiewicz, 2008; 2011; Kimmel, 2017; Schmitz \& Kazyack, 2016). These narratives fail to consider the contexts within which such violence was perpetrated and that VAWG is disproportionately a one-sided problem - whereby women are victims and men are perpetrators (Banet-Weiser, 2016; Citron, 
2012; Jane, 2014).). A key factor ignored by Incels (and other MRAs) is that underlying the perpetration of sexual violence is the social and structural context of the gender hierarchy - a "historically constructed pattern of power relations between men and women and definitions of femininity and masculinity" (Connell, 1987, pp. 98-99). Leaving a violent relationship is a practise that occurs over time. Individuals often go through a process of leaving and re-entering multiple times before ultimately ending the relationship, therefore should be understood as an ongoing process of leaving an abusive relationship (Kirkwood, 1993; Merrit-Gray \& Wuest, 1995). Victim-blaming women for the violence perpetrated against them ignores the complexity of victim/survivors leaving the violent situation (see: Eckstein, 2011 for greater discussion). In emphasising that women are the gatekeepers of sexual selection, and that any such violence they experience within a relationship is their own fault, Incels' reproduce harmful victim-blaming narratives that place blame on women for staying with a violent partner, whilst ignoring the pervasive and complex structural issues at work.

Other explanations for this crime advanced by Incels cast violence as natural for the biological male, noting that "[d]omestic violence is natural and should be done by all husbands and partners of women." Other comments also framed the violence in racial terms, where one user suggested "let's be real... niggers are going to kill each other no matter what. Degenerate criminals in USA: niggers $>$ hispanics $>>>$ whites $>>>>>>>>>>$ asians (rice/curry)." Such conceptions perpetuate longstanding media tropes of ideal victims and offenders. They also refer to out-dated genetic determinist understandings of race and crime. Harking back to biological positivist criminological accounts to explain the 'born criminal', such explanations evade structural analysis of the intersections of gendered, classed and ethnic inequalities. Such racist narratives that focus on stereotypical and negative tropes are understood by Levchak (2018) as microaggressions and cause harm to targeted audiences, thus reinforcing racial oppression. These discussions also reproduce the ideas discussed in Chapter 4 of the 'real man' as violent and aggressive, Black and Brown men as hyper-aggressive and violent, while viewing Asian men as effeminate and unlikely offenders (Greig, 2019; Levchak, 2018).

At first it appeared that the victim-blaming and racist narratives are neither reflective of postfeminist or backlash narratives within Jordan's (2016) typology with no direct references to (post)feminism at all. Incels analysis of such an event sidesteps structural evaluations of gender and power in favour of individualist understandings of their victimisation that centre on their own agency to leave a violent situation. These gendered understandings of violent crime cast male-violence, IPV and sexual violence as 'natural' biological urge. Such narratives also 
effectively naturalised black and brown men's violence, evading critical analysis of such violence. By utilising such 'scientific' and 'naturalistic' theories of violence, Incels both depoliticised the gendered nature of IPV, and its intersections with other forms of inequality. The framing of men's violence as natural by implication cast the victim as responsible for her experiences of IPV. Furthermore, through the 'de-gendering' logics of a postfeminist society of which Incels subscribe (Ging, 2019) Incels have created the space for the framing of the victim as entirely culpable for her own death. The implicit invocation of the genderless neoliberal subject silences the gendered nature of violence against women and girls, as well as normalising essentialist understandings of men of colour's violence that centres on media and cultural tropes and stereotypes (Levchak, 2018).

Victim-blaming, dehumanising, and othering narratives have potentially powerful and broad implications. Munn (2019) describes the process in which dehumanisation is used to foster racist attitudes within Alt-Right initiates. Incels utilise victim-blaming narratives to distract from the structural roots of social issues such as VAWG. Vetlesen (2005) has shown how such dehumanisation and narratives of 'othering' have worked in extreme cases to establish the foundation for the mistreatment (genocide) of certain groups throughout history. Vetlesen (2005) highlights how people pay forward the hurt and victimisation that they experience, which makes them feel in control of their victimisation. They then work to repurpose such anger onto some less powerful or marginalised group. Through misogyny and racist tropes, Incels reify categories, and creates distinctions between in and out-groups (more in Chapter Six), which dehumanise and 'other' groups who are lower on the perceived social hierarchy (Bael et al. 2019). Critical analyses of the roots of social problems are curtailed in favour of laying blame and instead blamed on the victims who are cast as lacking in responsibility.

In short, such narratives align closely with postfeminist and neoliberal logics, particularly in the depoliticisation of gender and the bio-essentialist explanations of gendered violence. Postfeminist logics that frequently centre on individualist 'scientific' understandings allow Incels to ignore structural examinations of gendered power dynamics. Within such a postfeminist culture, men within the manosphere utilise memes, inside jokes and other wellrehearsed arguments to silence critiques of gender inequality. As Jordan (2016) notes, however, the very nature of participating in such 'group-think' suggests somewhat of a collectivist politics. Yet such collectivist politics were communicated less in terms of the postfeminist discourses of the MRA groups of Jordan's (2016) study, and more frequently centred on the narratives of backlash: an understanding that significant gender inequality does exist, but it is 
men who are disadvantaged. The remainder of this section, therefore, focuses on such narratives.

Members of the Incel communities of Rreddit and Incels.co discussed backlash narratives frequently. These narratives were articulated through larger discussion threads as well as smaller comments within seemingly non-related threads. For example, the comment "Good morning sirs, just a reminder: we hate women!" was used frequently to derail critical discussions of gender or feminism. Larger discussion threads centred on three central narratives: feminist encroachment on male spaces; male problems (caused by feminism) and; the breakdown of societal norms and values because of feminism. ${ }^{24}$

The first backlash narrative centres on the idea of feminist encroachment on male spaces. It is argued that Incel forums are receiving large numbers of women who are posting and engaging with content within the public forums. In one thread titled "this is a male space", the top user opines:

Im seeing a sudden influx of women posting dumbshit comments on here, so I just thought I'd remind you all that this is a male space. We created this space for ourselves, and we don't want you here so fuck off... You have the government by the balls with a massive social movement dedicated to giving you access to every other space in existence. You have invaded every workplace, every community, every medium of entertainment that men created for themselves, and you have made them all worse and less functional. Why can't you just leave us the fuck alone in the one place we have left?

This user's plea for women to exit 'male spaces' mirrors the backlash seen in the 2014 \#Gamergate saga. Responding to the steady increase of women gamers (Casti, 2014), and the perception that game designers were corrupted by a feminist conspiracy to destroy videogames (Chess \& Shaw, 2015), users from Reddit, 4 and 8Chan, and other online spaces rallied together and coordinated doxxing attacks on feminists involved in speaking out against sexist gaming cultures (Massanari, 2017). This highlights a larger trope of new MRAs and Far- Right narratives that justify networked harassment by constructing feminism and women as villains, and men as their victims (Greig, 2019; Marwick \& Caplan, 2018). There is a high possibility that those individuals who were involved in \#Gamergate are also involved in Incel forums. The

\footnotetext{
${ }^{24}$ Many more narratives could be revealed within such a large data set, but I have chosen three levels of 'backlash' narratives in order to provide a richer commentary on the discourses pertaining to antifeminism.
} 
following comment is a response to another user who suggested that Incels and gamers are the most oppressed group within modern society.

You're probably joking but, gamers were genuinely bullied into becoming a political group... Game designers are intentionally going out of their way to make games less enjoyable for their loyal fans (by forcing narratives, squeezing women into combat positions they historically almost never occupied, removing sex appeal for the sake of removing it)... They were told by random feminists who didn't even play video games, and their journalists who control the companies with public pressure, that the things they want to see are immoral, and that they are being turned into sexists by the media they enjoy (whether they are aware of it or not). It's yet another male space corrupted by women and their self-centric ideologies, and it is no longer allowed to be a male space.

This highlights the fluidity and cross-pollination of antifeminist ideas throughout the manosphere (Ging, 2017). Such narratives consistently frame feminism and 'PC' culture as the oppressive to men, and constantly encroaching on spaces that were 'traditionally' White, maleonly "geek" spaces (Banet-Weiser and Miltner, 2016). As women and people of colour increasingly participate in such environments, backlash appears to be a common response. Incels 'feminist encroachment' narratives also mirror the MRA narratives observed during \#Gamergate that argued games and gaming spaces needed to be "safe spaces" for males in order to evade the alienation caused by feminists migrating to formerly male spaces, demanding to be accommodated (Futrelle, 2014).

The second backlash narrative that Incels consistently referred to was feminist-caused 'male' problems. The central MRA concerns of the 1980-2000 era saw backlash against feminism's perceived attack on fathers through family-law, as well as MRA challenges to anti-violence policies that they considered discrimination against men (Dragiewicz, 2008; 2011; Gottel \& Duton, 2016; Kimmel, 2017; Maddison, 1999). MRA's in the digital age are now largely focused on framing men as victims of false rape complaints and rape culture as a feministinspired moral panic, alongside other individual-level problems (Gottel \& Duton, 2018). These narratives are congruent with the pre-digital era Men's Rights movement who also advocated for such issues. Contemporary Far-Right forces have also been shown to deploy such discourses around the globe that mythologize the nation-family as under siege by feminist progress (Greig, 2019; Galasso, 2019). 
Incels constantly discussed male-problems as being caused by feminism. For example, a thread highlighting research that suggested men who are involved in online dating are at higher risk of eating disorders, bulimia, anabolic steroid use, fasting for weight loss contained virulent feminist-blaming narratives. It was argued that "despite common portrayal of eating disorders as 'female issues", men are more likely to experience dating-related pressures. Another user shared a popular sentiment "[t]here's nothing left to say. We're living in a fucking clown universe. Male problems are swept under the rug, or worse, portrayed as female problems. FUCK BEING BORN MALE." The users ultimately cast blame on women, and by extension, feminism for allowing women to have a larger degree of freedom, which has resulted in the perceived reduction of male power and privilege within society: "This is what men have been reduced to in 2019. It's a fucking sad day for our gender. Women are to blame for men's suffering."

This narrative was almost constant in threads discussing male health issues, including early death and suicide in both forums. Male suicide and other issues such as loneliness and addiction are significant public health challenges in advanced economies, particularly as the rise of online social spaces, gaming and pornography are particularly encompassing of millennial men (Yarrow, 2018). However, through its various explanations, the constraints placed upon men to perform a stoic, unemotional masculinity is among the most supported causes of men's pain. Men who conform to masculine norms have been shown to be less likely to seek help for mental health problems (Gorski, 2010). Incel discourses consistently exploit the crisis to blame women and feminism for men's issues.

This is seen directly within a thread on Incels.co, mourning the death of a niche YouTube personality who was well known for misogynistic videos posted from his mother's basement such as "I hate women", "women are not worth anything," and "life sucks". Incels used this opportunity to reflect on feminist-caused male problems. For example, one user asks the forum to "Imagine the kind of tortuous and lonely life this man lived. Truly a crime that society and women are responsible for." Another user reflects "He is a victim of our times. Times in which a man in the West is considered as somebody unworthy to be happy. Millions of other men will die too. There is a war going on against us." The Far-Right also plays on such "men in crisis" narratives to encourage sentiments of anger, to advocate ethno-nationalism, and to fracture attempts of social justice (Galasso, 2019, p. 105). 
The scope of feminism's supposed misandry is extended to the breakdown or decline of the 'West'. In these final examples of Incels' backlash narratives, feminism and women's move into the workplace is argued to have resulted in economic anxieties and a lack of opportunities for young men (see also Chapter 4). Instead of noting the social and economic shifts of many Global North economies to neoliberalism, the deindustrialisation of much of the labour force, globalisation and the slashing of social safety nets (Messner, 2016), Incels cast women and feminism as culpable for a precarious work environment and a turbulent, inflated economy. One user suggests:

"The West is now a nihilistic, hedonistic, materialistic, degenerate civilization of which hides all these traits by simply slapping on a 'freedom and progress' sticker on the front." To which another user argues, "The common link between all these issues is surely feminism, although it is cause and effect at the same time...Femoid $((($ independence $)))$ is always a very costly mistake. ${ }^{, 25}$

Further, Incels regularly assert, "the family system has disintegrated because of feminism" which is argued to also be coupled with "the breakdown and collapse of community is directly tied to the collapse of the family." One user suggests that "socially, we've become increasingly atomized... two things that have contributed to disintegration of the community is multiculturalism and individualism caused by feminist activism." This also mirrors Alt-Right and Traditional Conservative narratives that centre on calls to reassert 'traditional Christian family values' (Greig, 2019; Green, 2019). These issues are congruent with the broader men's rights politics, and hold continuity with the Mythopoetic Men's Movement that centre on the role of single mothers and the lack of father figures in causing a society filled with 'feminized men', with an Incel in a separate thread arguing:

Fucking women feminized most of the men and now [we] need mass migration to get those uncucked violent offenders that get their pussy wet. What happened to all the strong of character men that had self-control but were not pushovers? Guys today are either soycucks, limp pussy-whipped foid worshippers or violent impulse lunatics that don't

\footnotetext{
25 Triple parentheses (also, (((echoes))) or coincidence markers are used to highlight those of Jewish ancestry. Users of triple parentheses allege that triple parentheses highlight how much control Jews have over the world. (See: https://rationalwiki.org/wiki/Alt-right_glossary\#.28.28.28.29.29.29). The reference to "Femoid (((independence) $))$ " simultaneously blames Feminism and the 'Jewish Conspiracy' for progressive gains in women's rights, and its efforts to demasculinize white men.
} 
understand the term restraint. Fucking shit man, single mothers raise weak men or future prisoners. Feminized male - weak willed man. Feminized male mind - impulsive nut job that lashes out at everything \& never thinks about consequences of any actions. Masculine male - inner strength, composed. Masculine male mind - just because I can break his jaw doesn't mean I actually do it unless I have to, balance \& reasoning in every decision.

Incels are right to discuss important issues such as male suicide and the social/sexual alienation that they experience, as these are clearly troubling issues for not only men, but for wider society. However, in framing these and other broader social problems as the fault of feminism/ists, they are simplifying the complexities of such issues that require broader structural examinations of power. Many of the problems that Incels equate to feminism/ists are more broadly related to shifts in economic and social conditions that are outside of the control of the collective agency of feminists and women globally. As Ging (2017; 2019) has highlighted, MRA concerns have moved from collective concerns regarding law and structural changes for fathers' rights and divorce laws, to casting women and pro-feminist men as implicit in the disenfranchisement of the modern, White male. Such discussions centre on a scale of feminist encroachment in formerly male spaces such as gaming and internet cultures, to narratives blaming feminism for legitimate, yet misdiagnosed 'male' problems, towards a total conspiracy of the 'breakdown' of traditional values within society by the 'feminization' of men. Such backlash narratives centre on the unifying solution of reasserting patriarchy:

The feminist liberation that makes you sad and alone is virtuous, but the patriarchy that gave you meaning and family is oppression. The natural state of womyn is to perpetuate degeneracy and evil at every opportunity, and it is only the civilising influence of patriarchy that makes them human.

\section{Summary: Why blame (post)feminism?}

To understand such backlash and virulent anger of Incels it is appropriate to return the defining paradox that Incels grapple with: that men hold institutional power in a patriarchal society, yet most of these men do not feel very powerful. Within a postfeminist society where feminism has been thrust into the realm of the cultural, these men do not experience the power and privilege that they have been promised (Kimmel, 2014). The postfeminist culture and sensibility, as well as the rise in popular/celebrity feminism, on the one hand has created the 
conditions for mass consumption of a professionalised feminism. Conversely, it has also appeared to create the conditions where feminism is vulnerable to attack from its antithesis virulent misogyny and antifeminist men's rights politics. Following O'Neill's (2015) calls for masculinities scholars to research how the context of postfeminism has impacted men and masculinities, I have argued that Incels subscribe to the neoliberal and individualist/essentialist understandings of gender believing in a gender-equal society. This obscures the structural examination of gender and the other intersections of power and privilege. Backlash against feminism and multiculturalism then makes sense within such a cultural context that stresses that feminism has achieved its goals. Incels certainly do not feel that they are powerful, thus any perceived feminist gains exacerbate the sentiments of anger, resulting in vile misogyny and sometimes, lead to extraordinary violence events and calls for a 'beta-uprising.'

Threats of a 'beta-uprising' cannot be merely dismissed as the collective venting of anger alone (Ging, 2017; Nagle, 2016). Backlash was presented in terms of the negative view of feminism, casting feminism/ists as evil and actively disadvantaging men. Antifeminist discourses suggested a collective politics; however, this was rarely found to hold any real collective organisational power. It was, however, congruent with narratives that mirror Far-Right masculinity in crisis narratives (Greig, 2019). The sentiments of frustration and anger that Ging (2017; 2019) discussed were observable; there was also a strong sense that women's equality has resulted in a crisis of masculinity and the invasion of male-dominated spaces. This is argued to be harmful to women as the reassertion of patriarchal gender roles is cited as a 'civilising process'. Rather than addressing the concerning aspects of the neoliberal economic order and larger structural problems, Incels frame their political anger towards women, feminism and multiculturalism and evade intersectional analysis which may relegate their perceived oppression within a society that they wish to understand themselves as the oppressed. In the next chapter, I examine the overarching ideology of the 'Black Pill' that carefully selects academic studies and reinforces Far-Right narratives that exacerbate such sentiments of anger expressed by Incels. 


\section{Chapter 6 - 'The Black Pill'}

Incels are a danger to western society, because if we manage to blackpill more low value men, it could destroy this rotten civilization in its current form. Just imagine what is going to happen when more men realize that this society is just set up only for Chad and women and that they have no real stake in society. This knowledge could eventually spark a revolution against this degenerate (((society))) and lead to its downfall

As briefly discussed in Chapter Four, the Incel worldview is based on a categorical structure conceptualised as a rigid, three-tier social hierarchy that is exclusively based on appearance, where a minority of 'alpha' males and desirable females head the top, a majority of average-looking 'normies' follow, and a minority of physically unpleasant "Incels" are stuck at the bottom. This structure is common among more 'moderate' manosphere groups who consider "lookism" as the organising principle of social life (Papadamou, et al., 2020). ${ }^{26}$ However, what is specific about Incels is their belief that such a hierarchy is immutable, categories are impermeable, and no one is able to move from one to another. Baele et al, (2019) highlight that a belief in such rigid and unmoveable categorical structures is a key feature of extremist worldviews, and this is achieved within Incel groups through two main linguistic practices that the first two sections of this chapter examines. This first section utilises thematic data to understand the extremist worldview of the 'Black Pill', firstly by demonstrating aspects of the Black Pill worldview that 'naturalise' categories using biological arguments. The second section will discuss the 'radical dualism' of depictions of in and outgroups. These 'dualisms' focus intensely on points of difference within the out-group, which I will argue makes ideologically structured attacks on out-groups both about revenge for their perceived victimisation, as well as a group bonding mechanism for Incel members. Finally, this chapter shows that by using the Black Pill philosophy, Incels radicalise new members through the normalisation of ideologically structured hatred of women, with the end (intended or not) of producing a form of 'stochastic terrorism' as conceptualised by Clover (2019).

\footnotetext{
26 'Lookism' refers to prejudice or discrimination based on physical appearance and especially physical appearance believed to fall short of societal notions of beauty.
} 


\section{Unpacking the Black Pill}

As described in Chapter Two, several authors have discussed the Red Pill philosophy, which "purports to awaken men to feminism's misandry and brainwashing” (Ging, 2017, p.3). The analogy draws on the 1999 film, The Matrix, in which the protagonist Neo is given the choice between taking a red or blue pill. The blue pill represents disconnecting from reality into a delusional, agreeable world, while the red pill awakens him to reality and its hard truths and a commitment to fight back against it. Using the analogy, manosphere groups such as Pick Up Artists (PUA), MGTOW and other politically exclusionary groups such as the Alt-Right believe that the red pill allows men to manipulate the world to their advantage (Bratich \& Banet-Weiser, 2019). Incels have introduced a third, a Black Pill, that if metaphorically swallowed, makes one aware of the unchanging nature of reality: that the world is purportedly stacked against 'low-status men' in favour of women and alpha males; that there can be no personal solutions to systemic oppression, and the world was, is and always will disadvantage men who are 'genetically inferior'; and, finally, that women are inherently wired to prefer men with particular physical features over others. Baele et al., (2019) highlight that this is a crucial difference between the Incel worldview and less misogynistic forums within the manosphere, and that those who believe in the Black Pill understand that it is impossible to escape the social hierarchy that excludes them. The Black Pill is described on the Incel.co forum as:

...about understanding the fundamental nature of human social and sexual behavior, especially female mate choice. In opposition to mainstream self-help advice, the blackpill highlights the role of largely immutable traits in social and sexual exclusion. These traits include physical attractiveness, stature, muscularity, race, personality, ability, health, neurotypicality, social and economic status.

The internet forums of r/Braincels and Incels.co operate as ideologically rich information hubs for novice 'Black Pillers', who have likely encountered the milder Red Pill philosophies elsewhere on the internet. As suggested by one user, "It starts by browsing PUA forums, 4chan as a teenager, then stumbling across the ideas of the red pill. Later, you realise that for us genetically inferior men, we are doomed. Swallow the black pill, find out the truth." The truth, as argued by the Black Pill, is that "[s]ociety is set up for Chad and women only. The world is, was and always will be stacked against 'genetically inferior' men." In this way, Incel initiates 
move through the doctrine of the red pill gradually towards the more extreme worldview of the Black Pill.

Incels' revitalisation of genetic determinism is firmly centred within post-feminist neoliberal and individualist subjectivities (Chapter Five), and is chiefly concerned with theories of 'alpha' and 'beta' masculinity (Chapter Four), and hetero-normative rituals of seduction and mate selection (Ging, 2019). For Incels, "your life is in your looks"; put differently, physical attractiveness is a key determinant for sexual, economic and overall life success. Bael et al., (2019) argue that by utilising the Black Pill as an overarching philosophy, differences between the in-group (Incels) and out-groups (Chads, Stacys, normies) become reinforced, resulting in the creation of a sharp distinction between the in-group and its out-group oppressors. These beliefs help to form an ideologically-centred justification for demonising and dehumanising their targets in online and (occasionally) offline attacks; they work as an attempt to grasp the economic, political and sexual power that they feel entitled to.

Incel forum users regularly post statistics and 'scientific' data to prove the inescapability of their predicaments, which Ging (2017) has termed a sense of "turbocharged genetic determinism" (p. 13). Such beliefs are influenced by the PUA or 'seduction community', which are a series of blogs and websites aimed at teaching straight men 'pick-up' strategies based on evolutionary psychological concepts. For example, the notion that men need to dominate, and that women's natural desire is to be submissive despite their protests to the contrary, are an underlying feature of such communities (Ging, 2019). A core concept that such communities hold in common with Incels is that of 'sexual market value' (SMV), which utilises evolutionary psychological and neoliberal economic principles to explain sexual selection (Ging, 2019; O’Neill, 2015). Baumeister and Vohs (2004) argue that economic principles are present in the context of a cultural system where men and women play different, yet distinct, roles in selecting sexual partners. Baumeister and Vohs (2004) contend that men and women resemble roles of 'buyer' and 'seller', with men typically seeking to acquire sex from women by offering other resources in exchange. Incels also argue for a similar economic market regarding sex, where attractive and wealthy men hold more bargaining power in heterosexual exchanges than men who are of lower value in the sexual economic market. In a thread discussing attraction, this user highlights this 'hard truth' of the Black Pill:

Being attractive is what gets you laid. Without attraction, you can't have sex (and if you do... it's going to suck). It has fuck all to do with personality. Being blackpilled means you 
have the awareness to avoid the ills of being a beta-provider. ${ }^{27}$ You avoid the fakeness, the charade of a relationship with a woman that has no mutual attraction and is only with you for your money, and this is what I'm thankful for. You can't fake attraction. I may be unattractive, but I'm not a fool. Thank you, black pill.

Incels' fascination with physical attractiveness is directly linked to their belief in the worldview of the Black Pill and is frequently used as a justification for their sexual poverty. Incels often post comment threads to judge their own levels of perceived physical attractiveness within the sexual marketplace (Chapter 4). Reviewing evolutionary biology and psychological studies, and recently published data from dating sites such as Tinder and OkCupid, Incels interpret such research to explain their collective predicament of involuntary celibacy:

Evolutionary psychology explains why we are predisposed, or programmed, in certain ways. These underlying factors could be seen as major contributors to the existence of Incels. Many of the theories of evolutionary psychology, which relied heavily on selfreported and observational information, are now being supported by data gathered from dating services such as OkCupid and Tinder.

Incels believe that the combined knowledge of reviewing evolutionary psychological studies and their own, self-reported experiences has given rise to the ' $80 / 20$ rule'. This application of the Pareto principle suggests that $80 \%$ of women desire and are competing for the top $20 \%$ of men, while the bottom $80 \%$ of men are competing for the bottom $20 \%$ of women. ${ }^{28}$ An Incel.co user explains the Incels' predicament:

No shit $80 \%$ of men aren't virgins. But most men will have to bust their assess off and take scraps to get laid with any consistency. Men who can't (autists) or won't (volcels who demand a fair looksmatch) do this generally won't have foids available to them even if they are 7 s physically, unless they excel in some other field. ${ }^{29}$ Foids like

\footnotetext{
${ }^{27}$ A 'beta provider' refers to a man who works to financially support a woman who does not love or respect him, and is not sexually attracted to him.

${ }^{28}$ The Pareto Principle states that, for many events, roughly $80 \%$ of the effects come from $20 \%$ of the causes. The term is used in sales and business management circles, where it is thought of as an axiom that $80 \%$ of sales come from $20 \%$ of customers.

${ }^{29}$ The term 'autists' refers to individuals who possess autism-spectrum disorder characteristics and is used by Incels to highlight individuals who possess undeveloped social skills.

The term 'looksmatch' is someone from the opposite sex that is an equal or relative tier of attractiveness within the 'sexual marketplace'.
} 
men who stand out (in a 'good' way), and by definition that average man doesn't stand out.

The application of the Pareto principle to the arena of sexual selection has also been combined with the evolutionary psychological concept of 'hypergamy', which is understood as the phenomenon of women selecting a 'higher-status' male for mate selection (i.e., those 'alpha males' at the top of the sexual hierarchy).

The invocation of such theories to explain Incels' lack of sexual success works to explain women's decision-making based on their 'natural urges', and in doing so removes their individual agency. Such beliefs work to dehumanise the entire collective group of women, presenting them as only capable of simple, 'natural' emotions such as a primary drive towards sexual desire and reproduction. Incels' view women as holding the primary decisionmaking powers in the Darwinian sexual-economic market, stressing their 'animalistic' desires while casting moral judgement on them. Moral judgements over women's agency are utilised as tools to shame women, which creates a target for abuse when their sense of entitlement is threatened. The concept of hypergamy is also used to shame women for their sexual expression. This user expresses frustration at the supposed inequality of the sexual marketplace that assures women a long-term partner in the future.

Today women get to ride the cock carousel and still have a beta provider waiting for them at the end of it all, rather than having to immediately settle for and pair up with said beta provider in earlier years. ${ }^{30}$

Women being presented as the gatekeepers of sexual selection is not a recent phenomenon in psychology, and it has been highlighted that sex is a female resource that is culturally valued more than male sexuality. (Baumeister \& Vohs, 2004; Symon, 1979). The idea of female sexual gatekeeping is utilised heavily in Black Pill discourses to shame women's free choice for sexual selection. This provides Incels with ideological justification for expressing collective anger at women for not choosing them as sexual partners. It also creates the rationale for the reassertion for 'traditional' gender roles by casting women as irrational, and biologically driven, so therefore they should be under the patriarchal control of men. For example, as discussed in Chapter Four, Incels fixate on height and other salient physical traits as key components of

\footnotetext{
${ }^{30}$ The term 'cock carousel' refers to the belief that young women (16-25) have sex with as many 'high quality' men as possible, moving from one to another without thought. The reference to 'beta provider' is the idea that after riding the 'cock carousel' women will settle down with a beta-male who can provide her a comfortable life.
} 
sexual attractiveness. Here, the user utilises a misquoted 'law' in Robert Briffault's (1931) book to argue that in modern society, women choose a sexual partner with the highest possible benefit, and that women only view men as reproductive and status-improving 'objects'.

Briffault's Law maintains that the 'female, not the male, determines all the conditions of the animal family. Where the female can derive no benefit from association with the male, no such association takes place.' Any physical trait (height, ugly facial feature(s), deformity, that decimates a man's ability to provide a woman with social status via sexual association makes him a failure of a man, a "creep" or a "loser" in the mind of that woman. Height is measurable and very noticeable so it seems more important than it is. If a woman feels any aspect of a man's appearance ruins his ability to give herself status in the eyes of her peers/social group, then he will be rejected. Height is just one factor in this, but it is not the only one. Height, however, more than any other trait because of the fact that it is not subjective, proves how women actually view men: as status objects.

However, the sentence within Briffault's (1931) book never quoted it as a law, nor related it directly to human behaviour. In fact, Briffault was making a stark comparision to the patriarchal human family-unit and the social organisation of species within the animal kingdom:

The facts above illustrated show that the relations arising out of the reproductive functions, which constitute the only analogue of social relations to be found in the animal world differ conspicuously from those generally connoted by the term 'family'. That term stands, in the tradition of civilised societies, for a group centring around the interests, activities and authority of a dominant male. The husband is the head of the family; the other members of the group, wife and children, are his dependents and subordinates. The corresponding group arising out of that reproductive functions among animals presents no trace of that constitution. It consists of the mother and her offspring (Briffault, 1931, pp. 22-23).

Incels' uncritical, and incorrect reading of Briffault (1931) and their sharp biases against women coalesce to create and reinforce misogynist readings of social phenomena to argue for their perceptions of women's 'hypergamy'. 
Within the worldview of the Black Pill, one way to combat the biological imperative of women's hypergamy is by raising one's own Sexual Market Value. This can be achieved through one of two ways: by 'looksmaxxing' or improving one's physical characteristics in order to become more attractive (Chapter Four) or through the 'just be white' (JBW) theory. Aspects of JBW theory are based on cross-cultural studies of attractiveness and online dating data that suggest White men have a higher success rate than other ethnicities within online dating (Hergovich \& Ortega, 2017). This concept is succinctly explained by one r/Braincels user who argues, "race decides the probability of inheriting good features. With whites, the probability of inheriting facial projection (a prerequisite for attractive masculinity) is the greatest." Threads discussing the JBW theory often devolve into racist attacks on the non-white Incels of the group, discussing African American Incels: "If we analyse their racial features you would notice they have extremely dark skin, which is considered unattractive, huge flat noses which resemble those of chimps, curly hair which looks like pubes." The user goes on to evaluate their "positive traits", but poses the question "why are Blacks considered much higher on the racial hierarchy of SMV than say, Asians, Indians, brown Hispanics, or Middle Easterners? Especially because these races actually have more Caucasoid skull, lighter skin, and refined features than Blacks." Such genetic positivism is common within the Incels subculture and reinforces a racial hierarchy where White Incels reign supreme. This is a core feature of the Black Pill: the naturalisation and reification of categories of difference by utilising pseudo-scientific biological arguments. This feature also provides a 'scientific' justification for the problem of involuntary celibacy. Such narratives also mirror white supremacists' justifications for ethno-nationalist insurrection and the threat of barbarous masculinities of the ethnic other (Grieg, 2019).

Incels views of women and their supposed 'naturally' hypergamus behaviours highlight the mechanisms through which it produces both symbolic and real violence. Bael et al., (2019) recognise that the Incel worldview is based upon the past "golden age", a nostalgic time of a "patriarchal society where monogamy is the rule, traditional gender roles are accepted and followed, women and men marry early, and adultery is prohibited" (p. 13). During this mythologised era, it is imagined that all men had near-unencumbered "access" women as romantic partners, thereby reducing the competition for sex. Incel discourses also highlight a belief in a white supremacist racial hierarchy within such a "golden age" (Bael et al., 2019, p. 13). As discussed in Chapters Four and Five, Incels blame feminism and multiculturalism for the supposed 'declining position of men' in society. Frustrated with the prevailing system that 
is believed to be responsible for men's economic, social and sexual alienation, the Black Pill provides the conceptual ammunition to make calls for a 'beta uprising' appear as a legitimate course of political action.

\section{'Radical Dualisms'}

Incels incessantly depict out-groups (Chads/Alpha males, normies and women) as negative, whilst the in-group is portrayed positively (aside from discussions highlighting shared physical or mental 'deficiencies', as in Chapter Four). Torok (2013) argues that creating group polarisation is key to radicalising individuals, and the constant comparison of the in-group as 'just' or 'good' against that of out-groups strengthens the homogeneity of the group's worldviews. As Torok (2013) discusses, "The key to the success of radicalisation is the victor of one idea over another" (p. 8). We see this clearly in the rhetoric of the Black Pill in the policing of certain ideas over others. The Black Pill at once is utilised to polarise the outgroups, and simultaneously explain Incels place within the social milieu, thus strengthening bonds between group members through the collective venting of frustration and anger (Ging, 2019; Zimmerman et al., 2019). The following passage argues that the out-group (women and alpha males) are primarily concerned with physical attractiveness, which immediately contrasts with the user's comparison of Incels as morally superior and casting themselves as accepting of difference:

...just accept that the only thing that really matters is how you look to them so no worry about worrying about walking weird or acting like anyone but yourself. It's of no importance at best they will think of you as an ugly normal person, I don't see anything better about that than an ugly weirdo. Women and Chads are obsessed only with maximising a partner and friends holding high levels of physical attraction, while Incels just want to love and cherish someone who truly accepts and appreciates us.

Here, the Incel.co user presents Incels as romantic, and capable of providing unconditional love, whilst paradoxically they are presented as not driven by uncontrolled sexual desire based on attractiveness, a trait which the out-group is perceived to possess. Incels present the in-group as rational and morally superior, while portraying the out-group as animalistic and selfinterested - a trait supposedly not shared by the in-group. This contradiction is complicated by frequent comments that obsess over their desires to have a sexual partner and are further 
complicated by comments that advocate extreme violence against out-group members. "If I get the chance to kill I'm taking it. I don't care if I go down in history as the most evil and hated human being. I'd wipe everything out if I could. Stacys, Chad, normies - everyone." As Bael et al., (2019) highlights, the in-group is discussed as the only group capable of positive moral and pro-social values, and those who are black-pilled (thus intelligent or "high IQ") are the only people capable of seeing the truth about the social world. In other words, the in-group, invoking Black Pill principles cast themselves as intellectually and morally superior to all others. This strategy is also seen to be a feature in Islamic online extremist contexts and reinforces group cohesion, creating a shared set of norms and values for initiates (Torok, 2013; Awan, 2017).

As we have seen, women are frequently depicted as "low IQ", cruel, callous, and having simplified emotions (such as being guided by biological wiring). Incels frequently refer to women as "foids" or "femoids" or "roasties". ${ }^{31}$ This language casts women as 'other', lessthan and, at times not human. This makes ad hominem attacks against the group justified, whilst also renewing the in-group's sense of superiority over the out-group, such as in the following dehumanising passage:

Women aren't sentient. They have learned to imitate sentience with an uncanny resemblance. In reality, they are animals, animals wearing the flesh of humans who operate purely on instinct and their lizard brain. For some reason we evolved philosophically speaking, abstract thought and reasoning, but foids did not.

Discussing the Alt-Right journey through radicalisation, Munn (2019) highlighted that dehumanisation is a psychological prerequisite for violence, and shows that initiates learning the ways of the Alt-Right are never burdened with the idea of a 'human', a peer with a name, claim or story. For Incels, referring to women as "foids", "roasties", or "sluts" and claiming that they possess animalistic tendencies helps prepare Incels to justify violence against such monstrous archetypes. Bauman (2000) has observed that "dehumanised objects cannot possibly possess a 'cause', much less a 'just' one; they have no 'interests' to be considered, indeed no claim to subjectivity." (p. 96). Vetlesen's (2005) research which examined case studies of ethnic cleansing and genocide highlighted that dehumanisation has long been utilised as a strategy to galvanise support for lethal action against out-groups. This was also echoed by Munn (2019) who argues that through such dehumanising rhetoric, rights-bearing subjects are

\footnotetext{
${ }^{31}$ The term "roastie" is used to describe a woman who has had sex with more than one partner. It combines both body and slut-shaming practises, as it refers to a pronounced and elongated labia akin to that of roast beef.
} 
transformed into apolitical objects, clearing the way for rape threats, doxing, or occasionally in- in the case of Incels - extreme violence. By framing the out-group as responsible for Incels victimisation, such strategies of dehumanisation justify the threat of violence and misogynist attacks.

Furthermore, out-groups are depicted as portraying Incels as 'other'. Frequent posts in both forums centre on negative portrayals of Incels in media articles and opinion pieces. In response, this emotively triggers the in-group's sense of worth and heightens their sense of victimisation. Comment threads are filled with personal stories of perceived victimisation. In the following quotations, Incel.co members discuss a woman posting about avoiding her ex-partners in the context of living in a rural, small town. The Incels commenting on this post project their felt invisibility in the eyes of all women. "Roastie whore. She's not talking about 'people'. She's talking about Chad and only Chad and she can't wait to get dicked by the next one." A reply then suggests, "She doesn't see non-chads as 'people'. They're just objects who exist for her convenience, and protection in times of danger." A third weighs in "And as punching bags when she needs stress relief, sources of validation/money when she's depressed or broke, and objects of manipulation when she feels like being cruel and vindictive."

These kinds of exchanges reinforce in-group solidarity through a shared sense of victimisation by the out-group, which, in this case, the individual they are referring to represents the entire group of women generally. According to Torok (2013), by targeting the affective dimension of individuals through sharing moral outrage over perceived injustices by the out-group, group polarisation is reinforced, making attacks on the out-group easier and justified. Such exchanges are also exemplary of Papacharissi's (2015) 'affective counter publics', where digitally connected communities are discursively linked through the acts of emotional storytelling of suffering and common experience.

Bael et al., (2019) also demonstrated such in/out-group dualisms in their study, noting that the Incel in-group often acknowledges its "subhuman" nature. Bael et al., (2019) also reflect that despite challenging the view of extremist worldviews as made up of a positive ingroup facing negative out-groups, for Incels both in and out-groups are at times both negatively depicted (as seen in Chapter Four, when discussing Incels 'negative' genetic traits). However, Incels primarily present themselves as possessing positive traits and values that out-groups, particularly women, do not have. I suggest that the utilisation of the Black Pill as 'scientific' helps to protect against negative in-group portrayals, with 'Black Pilled' individuals accepting 
their position as 'natural'. For example, there was very little opposition to racist posts about non-white Incels despite frequent assertions of a racial hierarchy. Particularly for selfdescribed 'currycels,' there seemed to be a consensus that white Incels held a higher value on the sexual market.

The Black Pill creates such sharp distinctions to depict out-groups as morally inferior and ingroups as morally just and good. This simultaneously polarises and 'others' the out-group whilst strengthening the in-group's sense of solidarity. Grieg (2019) highlights that in FarRight communities, leaders use such radical dualisms to reinforce the hetero-normative patriarchal family, and thus present the nation-family as under threat. This normalises xenophobia, ethno-nationalism and authoritarianism. Incels frequently dehumanise out-groups, which Vetlesen (2005) and Munn (2019) have both highlighted as a psychological prerequisite for violence. Drawing attention to differences and hierarchy within the in-group were routine within the Incel forums, and as Bael et al (2019) discusses, the in-group is often acknowledged for its "subhuman" nature. The use of the 'scientific' Black Pill creates the conditions in which believers accept their 'position' within the SMV hierarchy as 'natural'. Polarisation between in/out-groups works to normalise and reinforce hatred, and therefore, online (and sometimes offline) attacks on the 'other', particularly women. Such misogyny normalising discourses are a key component to the radicalising process of the Black Pill ideology and are useful in understanding the raft of misogynist and sexist rhetoric within Incel communities. As Manne (2017) reveals, misogyny acts as a mechanism by which those threatened by female empowerment work to police women who step outside the status-quo, thus used it is used as a tool to reinforce patriarchy.

\section{Normalisation of ideologically structured hatred against women}

The online environment is an important context to normalise radical thinking (Awan, 2017; Torok, 2013). Users become attuned to the values and norms of the community through discussions and disciplinary techniques that 'normalise' the way users should think and act (Chapter Four). Like other online extremist groups (Green, 2019; Torok, 2013), Incel users who argued against the Black Pill 'tenets' were quickly policed when discussions breached ideologically acceptable boundaries. This plays a key role in mediating anti-Black Pill opinions, thus leaving little space for critical discussion. Munn (2019) describes three overlapping cognitive stages that alt-right initiates are nudged along throughout their 
radicalisation journey: normalisation, acclimation and dehumanisation. It is through similar cognitive stages that Incels, too, come to accept the Black Pill worldview.

Through normalising the 'pill' ideology, Munn (2019) demonstrates how discourses of humorous and ironic inside jokes (in the form of gifs, memes, clever references, language play, absurd juxtapositions and 'edgy' humour) normalises racism, xenophobia, and misogyny, providing the frame for alt-right values. Incels frequently invoke the 'science' of the Black Pill to suppress independent and critical thought from both outsiders (such as Incel Tears members) and insiders (Incel members themselves). This works simultaneously to normalise and police opinions for the wider Incel community.

Other studies of extremist groups show that online radicalisation also necessitates a form of self-isolation. This is discussed as a crucial stage for learning the social meanings and behaviours within the online forum environments (Awan, 2017; Torok, 2013). It is also a crucial stage of normalisation in Munn's (2019) radicalisation process. As discussed in the case of Elliot Rodger and other Incel 'Saints' (Chapters Two and Four), it is clear that social isolation is prevalent in Incel communities. These forums are highlighted as key sites for social interaction for many Incels, as one user laments:

No one calls me on my cellphone. I don't even know why I have one. I only use it as an MP3 player at the gym, and when the delivery guy calls me. Other than you guys, I don't really talk to anyone. The isolation is pretty hard sometimes. But it's better than talking to fake people.

The self-isolation that Torok (2013) and Awan (2017) described provides insulation from pervasive outside influences, especially alternative ideas and competing rationalities. Seeking out others with similar ideas and experiences, and by engaging with Incel forums and chatrooms, 'experts' in the Black Pill 'train' others by debating current events in reference to their own isolated and individualised experiences. Occasionally, when dissenting opinions challenge the beliefs of the Black Pill, they are either heavily down-voted (in the case of r/Braincels) or heavily debated using 'evidence' that is often taken from the cache of Black Pill information located within the 'Incel wiki' tab on Incels.co. ${ }^{32}$ Torok (2013) highlights that

\footnotetext{
${ }^{32}$ The 'Incel Wiki' and 'BlackPill' tabs are located on the top left-hand side of the homepage of Incels.co. These hubs of user-generated information make up a core feature of the 'scientific' arguments presented in Incels.co comment threads. It is publicly accessible and users often refer initiates to these tabs to learn elements of the Black Pill.
} 
during self-isolation, time spent in online spaces allows access to 'normalising' radical discourses. The normalisation of 'in-jokes' and the varied lexicon featured within Incel discourses requires users to develop a sense of 'disciplined regularity' (Torok, 2013) to understand the references, edgy humour and language play. This is crucial for the 'acclimation' stage where users are ideologically conditioned by the sheer volume of hatred against the out-groups (Munn, 2019). Ging (2019) also highlights that within the 'postfeminist' society (Chapter 5) gender politics for groups within the wider manosphere take place within the cultural realm of memes, inside jokes, and the edgy humour displayed within the normalising discourses of radicalisation argued within this chapter.

However, in conceptualising Incels (and other online radicals) retreat to the self-imposed online isolation described by Torok (2013), there is a risk of repeating the false distinction between the 'virtual' and 'embodied' nature of technology and the terrestrial world. This view fails to capture Brown's $(2006$, p. 277) suggestion of the nature of contemporary society as a "human/technical hybrid". The virtual/real binary is an ineffective reference point (Powell et al., 2018). As Ging (2019) has shown the configuration of technology, ideology and emotional appeals or calls to action are particular features of the wider men's rights movement of the manosphere and its fluid coalescence around collective political action (Papacharissi, 2015). In digital society, the nature of hatred and bigotry are technosocial processes, which result from ongoing cycles of technological co-production (Powell et al., 2018). These are mediated through, and afforded by, technological platforms such as Incels.co and r/Braincels, both of which are easily accessible with a smartphone. Such technology allows for continuous connection and social engagement with Incel discourses. Rather than the self-imposed online isolation proposed by Torok (2013), Incels are able to be in continuous contact with Black Pill culture and despite reporting being isolated in the offline world, actively engaged Incels are far from isolated within the online sphere. As Powell et al. (2018) have argued, "[t]echnology is not just revealing pre-existing bigotry within society; rather, technology and society are shaping one another, coalescing in emerging cultures and practices that simultaneously produce and reproduce hate based harms." (p. 121 emphasis in original).

The final stage of the radicalisation journey for Munn (2019) is the 'dehumanisation' stage. As discussed in the previous section, the dehumanisation and 'othering' of the out-group is a regular occurrence in the online echo chambers of Incels. Dehumanisation of the out-groups appear to be a common feature, and a way that Incels bond - whether it be through memes, 
gifs, short inside-jokes and wordplay or extended essays (Bael et al., 2019). Dehumanisation plays a role in creating a reading of the social world as in a 'crisis' not only of masculinity, but also of a 'crisis of beta masculinity' (Ging, 2019; Nagle, 2016). In discussing such crisis-narratives through humorous dialogue and edgy humour, to more serious and extensive essays, Incels collectively (re)produce comprehensive blame-attributing narratives, which centrally locate the actors responsible for the 'crisis of beta masculinity' and their own Inceldom. Subsequently, these narratives advocate for violent punishment for those who have hurt them (Bael, et al., 2019).

Incels frequently discuss previous attempts by the "Incel Saints" of Rodger, Lepine, Sodini, or Minassian and others to violently punish their perceived oppressors. Their admiration and support of violence is regularly expressed through the profile pictures of their pseudonymised accounts, as well as regular victim-blaming narratives such as "If only some foid would have fucked [Elliot] Rodger, then perhaps he wouldn't have gone ER on their ass." As shown throughout the previous chapters, many Incels show strong support for violence, particularly against women. Johnston and True (2019) show that hostile sexism and violence against women are strongly associated with support for violent extremism. Similarly, McCulloch, Walklate, Maher, Fitz-Gibbon and McGowan (2019) too have demonstrated this link in the context of lone-wolf terrorism, citing the failures of the Australian Police negotiators to acknowledge the prior intimate partner violence perpetrated by Man Monis during the 2014 Lindt Café siege as significant in their decision to raid the cafe. ${ }^{33}$ Rodger, Lepine and others shared a common history of violence against women and overt misogyny, and Incels regularly valorise mass-murderers who shared such misogynistic beliefs. Pain (2014) connects the intimate and structural dynamics of daily terrorism, such as domestic violence, to wider global terrorism. Both forms of 'terrorism' aim to exert political control through fear, trauma and violence (Pain, 2014). Incels' valorisation and participation in hostile misogyny is indicative of a form of both the 'everyday' and 'global' terrorism that Pain (2014) conceptualised.

\footnotetext{
${ }^{33}$ Monis had a long and documented history of violence against women. At the time of the siege, he was out on bail for 40 sexual offences committed against seven different women and being an accessory to the murder of his 30-year-old former wife and mother of their two children. (See: McCulloch et al., 2019 for a detailed analysis of the Lindt Café siege).
} 
For example, within Black Pill -related comment threads Baele et al., (2019) also cited frequent attempts to invoke uprisings and revolts, akin to the 'beta uprising' that Nagle (2016) discussed. This Incels.co user describes the chain reaction and purpose of such an uprising:

What I want to happen is for there to be a mass shooting/bombing every single day in every single city in the world, ideally targeted at politicians (and their lackeys), media conglomerates, and financial institutions. No one should be allowed to live in ignorance of how the other half lives. "Bring the war home."

The 'truth' or 'science' of the Black Pill was often used as a direct justification for calls for domestic terrorism and Incel uprisings, and the lives of the out-group are presented as inconsequential to the broader goals of punishing society for the 'crisis of beta masculinity':

Death is a pre-requisite of Life. Brown, White, Black, it doesn't matter. Death tends to happen. The thing about murder is that not only does it kill people... it also can change the course of history. Of course, I would prefer it if Women died. But death happens and I couldn't care less about what their ideology or skin colour is as long as people are dying before their biological death date, that's all I care about. Chaos, something that the Elites hate. It is something that I admire. Its final form is the Blackpill and no one can deny the truth of what we must do.

However, as Bael et al., (2019) have shown, such ubiquitous support for violence, whether it be in the form of the fantastical uprisings mentioned here, in lone-wolf terror attacks such as Elliot Rodger or the other "Saints", or the more benign forms of online violence, all provide a sense of catharsis for Incel members troubled imaginations. In the wake of the 2019 Christchurch Mosque attacks, and other high-profile lone wolf terror events during the data collection period, frequent posts discussing "kill counts" and debating the motives of the separate killers were observed. Memes depicting the 'Chad NZ Shooter' were posted to the Incels.co forum, valorising the number of women and Muslims killed in the attack. Continuities between the posts of such attacks centred on the number of women killed, maimed or injured. Such clear encouragement of extreme violence, particularly targeted against women is, as Reicher, Hopkins, Levine and Rathe (2005) note "legitimated as 'self-defence' against the perceived aggression of the target and atrocity is generally represented as a noble and even virtuous act” (p. 630). It is through such online discussions of intimate, yet chaotic violence 
on the scale of lone-wolf shooting, as well as the everyday forms of misogyny directed against women, that both forms of Pain's (2014) terrorism are connected in Incel forums.

These last examples are valuable as they clearly demonstrate the connection between Extreme Right Wing and Incel ideologies. They also thrust the continuities of everyday violence, and its extreme manifestations of violence in mass-shootings into the spotlight (Johnston \& True, 2019; McCulloch et al, 2019; Pain, 2014). The online forums of Reddit and Incels.co show the cross-pollination of ideas through memes, hyperlinking of articles and the overlapping interests that such cultures hold in common. These interests are underpinned through hardened misogynistic attitudes that call for the reassertion of patriarchal societies through the use of a style of militant misogyny (Zimmerman et al., 2019) and deeply embedded masculine gender constructions influenced by Far-Right narratives (Grieg, 2019). The co-production and sharing of common memes suggest that in digital society, hatred and bigotry are expressed through such technosocial networks and are not independent to one culture or forum alone (Powell et al., 2018). Such a manifestation of hateful rhetoric (and action) against gendered or racialised 'others' (the common out-groups) are explained and (re)produced through hierarchies of difference in an attempt to push-back against a 'new' globalised, multi-cultured world where previously many of these men likely would have felt more powerful and important than they do now (Grieg, 2019). Incels' extreme conclusions made from syntheses of carefully selected scientific studies and xenophobic, ethno-nationalist and authoritarian narratives work to educate initiates and provide them with an evidence-base of seemingly objective research that confirms their lived experiences of supposed subjugation by feminist misandry.

Even 'moderate' Black Pill beliefs retain an air of violence, pushing back against women's rights: "I wouldn't [commit murder] because it's not my goal. My vision is to blackpill men and uncuck laws so mankind will be free of delusion, limit women's hypergamy, and put women in their place." Here, corporeal violence is substituted for textual violence for the goal of reducing women's liberties. The Black Pill philosophy intends, at the very least, to reduce the rights of women (and that of non-White people) and their status in society by its reversal to an imagined state of a racialized, patriarchal hierarchy of the "golden age" (Bael et al., 2019, p. 13). The Black Pill is problematic in its unwavering support of violence against out-groups. Even if it does not inspire the mass-shooting events such as Rodger, Sodini or Minassian, these radical views oppress others in real ways in the invisible forms of everyday violence enacted against women and girls. As McCulloch et al., (2019) argue: 
The failure to see clearly the continuities between what is typically understood as private violence against women and lone wolf terrorism is part of an ongoing failure to take women's security seriously - a failure that also limits efforts to understand the risks of and respond effectively to lone wolf terrorism... Viewing these two forms of violence as existing on a continuum reframes violence against women as a form of everyday intimate terrorism, and lone wolf terror attacks as an escalation of extant tendencies within particular individuals (p. 447).

Munn (2019) highlights that the Alt-Right utilise mass communications such as easily proliferated memes and YouTube videos to incite random lone wolves to carry out violent terrorist acts, and cited Hawkins (2019) to observe that the absence of a formalised structure or parent organisation makes Far-Right extremism difficult to detect or disrupt. Munn (2019) argues that the rising frequency of violence and 'scattershot quality' of such violence can be explained by the idea of 'stochastic terrorism', which encompasses the notion that while violent attacks can be statistically predictable, they remain individually unpredictable. Like previous Incel attacks, Munn (2019) points out the individual nature of such attacks; the shooter chooses his own targets, location and specific motive. Such attacks, in the context of the Black Pill are too; they are dramatic, singular and look like "an individual killer obeying their own disturbance, their own nature or character or conscious belief" (Clover, 2019). However, as Clover (2019) argues, the actions of such individuals are an effect of the larger structure that reveals itself. For Incels, these structures reveal themselves at the nexus of the intersecting factors such as harmful (Far-Right) constructions of masculinity, broad economic changes leading to insecurity, social alienation, and the technosocial construction of echo chambers in which harmful worldviews exist unchallenged. Stochastic violence emphasises rhetoric that inspires small cells or individuals ("lone wolves") to commit acts of violence, while retaining deniability for leaders and groups (Biondi \& Curtis, 2018). The 'scientific truth' of the Black Pill ideology, therefore, is a 'truth' that makes it necessary for believers to blame, and therefore punish, women for their positions within society. Provocative rhetoric and ideology incite users to interpret the political and personal calls to action as they deem appropriate, resulting in a raft of harms from online misogyny to extreme mass-violence attacks. 


\section{Summary}

This chapter has suggested that the 'Black Pill' is an ideology of carefully selected and cherrypicked theories deriving from the disciplines of evolutionary psychology and biology, as well as psycho-economic 'neoliberal' principles, merged to form a confluence of misogynist and white supremacist beliefs. The Black Pill provides an explanation for Incels perceived disadvantaged position within society, while also providing an ideological scapegoat to target their collective anger (women). The Black Pill also works to provide justification for their oppression using techniques of group polarisation, which results in 'radical dualisms' that focus on sharp points of difference between in/out-groups (Awan, 2017; Torok, 2013). I argue that the Black Pill normalises misogyny and racism by using ideologically structured hatred against women specifically and non-Incels generally. Such an ideology is similar to, yet differentiated from, Far-Right rhetoric insofar as its constructions of a wounded masculinity, and masculinity in crisis narratives, are enhanced by an acute sense of self-loathing and nihilism, as well as extreme misogyny that is not as evident in alt-right circles (Grieg, 2019). This ideology, whether seriously intentional or not, is responsible for producing 'stochastic terrorism' through its radicalising and dehumanising narratives that cast users as responsible for their own individualistic interpretation of the radical Black Pill. 


\section{Chapter 7 - Discussion/Conclusion}

In order to understand the virulent misogyny espoused by arguably one of the most extreme examples of online antifeminist groups, this thesis examined the Incel subculture. First, I interrogated the structural contexts in which the culture derived by initially discussing Incels conceptions of masculinity, highlighting that Incels sense of masculinity simultaneously loathes and reveres 'Chad' - a caricaturised version of the hegemonic (Westernised) standard of masculinity. Incels position themselves as 'beta-males', a conception of masculinity that identifies itself as against feminism, political correctness and hegemonic masculine norms and that mirrors Far-Right constructions of masculinity (Grieg, 2019). However, my data revealed that despite railing against hegemonically masculine norms, Incels utilise a 'hybrid masculinity' (Bridges \& Pascoe, 2014) which valorises particular aspects of the masculinity of Chad, while attacking women and other subordinated or marginalised masculinities. Reflecting on Nagle's (2016) position, which rejected the notion that beta-masculinity is incongruent with the typical forms of hegemonic masculinity, I argued that it does, in effect, the same thing: it reasserts and celebrates a patriarchal ordering of society whereby men are positioned as dominant over women. This is directly observable in calls from Incels to reassert a patriarchy and restrict women's rights.

Incels' beta or hybrid masculinity and their sense of 'aggrieved entitlement' occurs within their view of society, which they perceive as economically, sexually and socially favouring women (Kimmel, 2014). As observed in Jones et al's (2019) study of the MGTOW group, this sense of victimisation and aggrieved entitlement is also at the core of Incels' group identity. Incels claim that feminism is at the centre of their sense of victimisation. They believe that women's empowerment has created the space for women to express their sexual agency, thereby, creating conditions where women are "picky" and innately superficial in choosing sexual partners based on physical attraction. Incels explain their broader sense of powerlessness in social and sexual terms due to their supposed genetic disadvantages. By not being able to conform to the athletic and conventionally physically attractive capabilities of 'Chad', Incels frame themselves as victims of both feminism and their own biology. Such concerns parallel pro-feminist concerns of the early Men's liberation movement that understood that the 'male sex role' was destructive and even lethal for men's health, emotional lives and relationships (Messner, 2018). They also mirror contemporary Far-Right gender constructions that are centred on threats to (White) masculinity from ethnic and feminised 'others' (Grieg, 2019). 
Within the contemporary manifestations of such gender politics, Incels call for the reassertion of patriarchy through organised violence.

Such calls for political violence have been pressed aside by some as the work of entertainment seeking trolls who enjoy the 'shit-posting' and railing against social norms (Nagle, 2016). Arguably, many Incels are likely harmless and merely engaging in such 'trolling' for reasons of self-enhancement within online echo chambers (Jaki et al., 2019) and to reassert their fractured sense of masculinity (Jones et al., 2019). However, such collective expressions of anger and skewed ideological beliefs are also responses to the social and sexual alienation that they so deeply experience. In fact, such beliefs have had very real effects, as in the case of Rodger, Minassian and others who have literally killed in their attempts to grasp power over women's bodies, over which they hold an intense feeling of entitlement.

Like other manosphere groups, the data revealed that Incels hold intense economic anxieties. This finding was in common with other studies, which have shown sexism, racism, and economic dissatisfaction to be related to the rise of extremist communities. Online communities, in particular, have been shown to provide fertile ground for the growth of such exclusionary politics (Grieg, 2019; Green, 2019; Odin-Shaw, 2019; Schaffner, MacWilliams \& Netea, 2017; Wayne, Ocean, \& Valentino, 2018). Incels growth has similarly developed alongside a broader 'backlash' against feminist action and social justice movements of the past 30 -years, whilst the socio-economic and cultural changes brought in by neoliberalism that have especially affected the White working class are largely ignored (Kimmel, 2014; Messner, 2018). These larger structural changes are rarely acknowledged by Incels themselves, who opt instead to blame feminism and multiculturalism for their broader economic anxieties.

For (Western) men particularly, these changes to waged work - which have previously been a central and defining place in the construction of masculinity - have become increasingly fractured due to deindustrialisation in the global north (Connell, 1999; Salzinger, 2016). As such, it is argued that the terrain in which masculinities are constructed has shifted profoundly over the recent decades. A linear progression from school into the workforce is no longer guaranteed, and many young people find themselves cycling in and out of short-term, precarious work (McDowell, 2019; Nixon, 2017). Regular waged work is no longer assured for men, thus challenging the traditional male breadwinner role (Grieg, 2019). In an increasingly feminised and multicultural neoliberal economy, Incels highlight their dissatisfaction and anxiety in competing for jobs for which they feel entitled to. Such fears of 
female (and racial) encroachment on 'men's work' and 'men's spaces' was found to be experienced as a threat to Incels' masculinity (Banet-Weiser \& Miltner, 2016). Therefore, feminism, multiculturalism and progressive 'PC' culture generally, are highlighted as a direct threat to Incels' sense of masculinity, and are routinely blamed for their social, sexual and economic alienation.

O’Neill (2015) has called for masculinities scholars to examine how the social and cultural context of postfeminism has affected masculine subjectivities and men's practices. In heeding the call, Ging (2019) posited that the mutually synergistic dynamics of neoliberal capitalism, the technological affordances of social media platforms and the cultural context of postfeminism have shaped contemporary online antifeminism. Discussing the broader manosphere, Ging (2019) argues that despite the Men's Rights Movement's (MRM) very real and occasionally explosively violent nature, it is incoherent and unconnected, tending not to mobilise publicly like feminist movements. Instead, within the Web 2.0 era, social media has allowed for an expansive array of cultural memes, inside jokes and other affectively charged tools to engage with gender politics. Ging (2019) highlights that for MRMs, memes and other cultural materials function as collective venting of anger, rather than nuanced political debate. The current study concurs with Ging (2019), demonstrating that for some Incels who have grown up within the 'ordinariness and everydayness' of a postfeminist neoliberal society that implies a 'pastness' of feminism (O’Neill, 2015; Tasker \& Negra, 2007), the recent renewed luminosity of feminism has created a perception that women are attempting to gain an unfair advantage. However, diverging from Ging's (2019) assessment that such movements are seemingly incoherent and unconnected, and therefore easily quashed, I have argued that the confluence of misogynist and white supremacist worldviews united within the Incel worldview represents a significant threat to society due to the random and chaotic nature of its outbursts of violence. This argument is put into even sharper focus when considering Pain's (2014) understanding of the continuities between everyday intimate terrorism and global terrorism; both have the intent of instilling fear and achieving political influence.

Incels' collective venting of anger centres around a defining paradox: that (white) men hold institutional power in patriarchal society, but most do not feel very powerful personally. Yet, structurally, the male gender still dominates. At the heart of the Incel ideology are hardened misogynistic notions of the need to reassert 'traditional' gender roles (Grieg, 2019; Zimmerman et al, 2019). Incel narratives such as Minassian's call for “Incel Rebellion” parallel white 
supremacists' calls for ethno-nationalist insurrection (Greig, 2019). It follows that for Incels who are frustrated with the prevailing system, feminist activism for equality exacerbates sentiments of anger, and results in backlash against feminism, women and those who support progressive causes. The current study concurs with the assessment of Zimmerman et al (2019) who argue that such a sense of social frustration, alongside the adherence to an ideology that promotes destructive, violent solutions, makes Incels dangerous actors both in and of themselves, but also increases the possibility of recruitment to other extremist sects. These exploratory findings highlight the importance of further research that continues to unveil the conceptual apparatus that underpins such groups of angry, young men.

Furthermore, Incels narratives and philosophies straddle other forms of the five key categories of online men's politics that Ging (2017) observed. Such narratives and cultural tropes centred primarily on women (and feminism) being gatekeepers of sexual selection and argued that women were to blame for their sexual alienation. Using Jordan's (2016) Feminism, Postfeminism, and Backlash Typology, it was shown that Incels narratives utilised simplistic evaluations of gender power dynamics and focused heavily on 'proving' that feminism was no longer necessary. Many comment threads depicted women as amoral subjects who were driven purely by their 'natural' biological urges for procreation. Incels used cherry-picked biological and evolutionary psychological studies to create complex arguments for their lack of sexual success, and to shame women for their sexual agency. Throughout Chapter Five, I demonstrated that the context of postfeminism has affected Incels by privileging individualist/essentialist understandings of gender that invoke the 'genderless' neoliberal subject. Due to a belief that women have equal and equitable rights in contemporary society, any feminist claims were routinely interpreted as women trying to gain an advantage over men. The invocation of the genderless neoliberal subject obscures structural examinations of power and privilege; privilege which many Incels undoubtedly possess. Backlash against feminism and multiculturalism, therefore, has originated within the cultural context of postfeminism that stresses that feminism as a movement has achieved its goals of equality (Banet-Weiser \& Miltner, 2016; Gill, 2017). This finding is also salient for the other key categories of online men's politics that Ging $(2017 ; 2019)$ discussed and is important in relation to understanding the gender politics of the manosphere. 
The Incel ideology routinely centred on an array of biological and psychological determinist theories known as the Black Pill. Incels understand the Black Pill as an organising principle of social life. It creates a rigid three-tier hierarchy primarily based on physical attraction, where a minority of alpha males and desirable females are located at the top, a majority of average looking 'betas' follow, and a minority of physically unpleasant Incels are stuck at the bottom. The Black Pill believes that such structures are impermeable, and that transcending one's genetics is impossible. Bael et al., (2019) highlighted that this belief in impenetrable structures is a key feature in extremist worldviews. A belief in the Black Pill represents an understanding that it is impossible to escape the hard boundaries of the social hierarchy that excludes them. Therefore, the Black Pill ideology implies that in order to transcend such seemingly impermeable boundaries, a violent retribution must take place against those who hold a higher place in the social hierarchy (for example, the 'beta rebellion' called for and attempted by Minassian and which influenced the 2020 Incel attacker in Sudsbury, Ontario).

The beliefs of the Black Pill, as Bael et al., (2019) acknowledged, work to produce and reinforce sharp distinctions between the in-group and the out-groups (Chads, Stacys, normies). This creates an incessant negative depiction of out-groups, whilst portraying themselves as morally superior. Torok (2013) highlighted that creating group polarisation is a key component used to radicalise individuals. This creation of radical dualisms also works to reinforce group solidarity and cohesion. The 'science' of the Black Pill is the base for which a shared set of group norms and values are created and reinforced. The Black Pill also further creates radical dualisms in the sense that the out-group is frequently depicted as portraying Incels as 'other', or worthless, furthering feelings of alienation or exclusion. Shared accounts of perceived victimisation and citations of evolutionary biology are utilised to trigger the in-group's already diminished sense of self-worth. This creates moral outrage against the out-group (women) of which the embers are routinely stoked through the invocation of a shared ideology of the Black Pill. The techniques of creating group polarisation and developing a shared set of norms and values that are policed by group members are also utilised by other extremist groups such as radical Islamist and extreme Right-Wing groups (Awan, 2017; Torok, 2013; Greig, 2019; Green, 2019).

The online environment is the vital context to normalise radical thinking and behaviour, and disciplinary techniques 'normalise' the ways users should think and act (Awan, 2017; Green, 2019; Torok, 2013). Munn (2019) described three overlapping cognitive stages that alt-right 
initiates are nudged along throughout their radicalisation journey: normalisation, acclimation, and dehumanisation. Incels parallel such a journey, and as Ging $(2017 ; 2019)$ highlighted, they engage with memes, cultural information and ideas that span the wider manosphere that shape and 'normalise' initiates behaviours and values. Future research may investigate the extent to which memes and other cultural references which work to normalise racism, xenophobia and misogyny span the manosphere generally. Such research could provide further insights to the confluence of misogynist and white supremacist worldviews. These important next steps could also provide insights to Munn's (2019) 'acclimation' stage, where the sheer volume of ideological hatred against out-groups successively conditions users to fear and react to existential crises invoked by the onslaught of hysterical fear-producing narratives of the 'other'. Incels time within the online environment creates a form of self-isolation that Awan (2017) and Torok (2013) discussed as providing insulation from pervasive outside influences and competing rationalities. The configuration of technology, ideology and emotional appeals or calls to action are unique and central features of new digital politics of sentiment (Ging, 2019; Papacharissi, 2015). These features combine to propel 'Black Pilled' users to demonstrate their hatred in individualised ways. For Incels, the self-isolation proposed by Torok (2013), is facilitated through continuous contact with Black Pill culture, whereby users are consistently indoctrinated with the Black Pill ideology (Powell et al., 2018). As Incels are self-reported 'loners', and isolated within the offline world, it was found that Incels major sense of communication and connection may well come from such online communities, exacerbating such insulation from pervasive outside influences.

However, Incels should not be thought of as a lone group of extremely unhappy men whose bigotry and dehumanisation of out-groups only occurs in isolation. As Ging (2019) and other scholars in the area have highlighted, Incels are part of a broader manosphere that share similar political ideas and values. Furthermore, as Pain (2014) highlighted, such global terrorism is intimately connected with the everyday phenomenon of domestic violence and patriarchy. Incels collectively repeat blame-attributing narratives that centrally locate women as responsible for a broader 'crisis of masculinity', and their own Inceldom. Incels ubiquitous support for violence is particularly troubling as the use of the 'science' or 'truth' of the Black Pill is used as a justification for violence against women and minorities. The interconnectedness of such violence is seen directly after lone-wolf violence such as the 2019 Christchurch Mosque shooting, the Dayton, Ohio and El Paso, Texas shooters and the most recent Incel attack in Sudbury, Ontario - with each of these attacks targeting women and ethnic 
minorities and justifying their attackers with similar hate-filled rhetoric. Memes and discussions about such attacks frequently commended and analysed the attackers' methods of destruction. This provides further evidence of the interconnectedness of the wider manosphere and the links to Right Wing extremism.

The frequent and hostile misogyny observed within Incel forums is also problematic. Incels frequently discussed support for previous "Incel Saints" such as Elliot Rodger, Alek Minassian, Lepine, Sodini and a raft of other lone-wolf shooters who represented Incel values. Such 'Truecels' were frequently valorised, discussed and supported for their murders. McCulloch et al (2019) demonstrated the link between lone-wolf terrorism and previous violence against women. Johnston and True (2019) also highlighted that hostile sexism and violence against women are strongly associated with support for violent extremism. The nature of Incel violence meets the requirement of the U.S. State Department's description, which defines 'terrorism' as "premeditated, politically motivated violence perpetrated against non-combatant targets by subnational groups or clandestine agents." Moreover, in 2020, the Texas Department of Public Safety have labelled Incels an "emerging domestic terrorism threat (p.3). The Incel ideology of the Black Pill should be considered as a form of violent extremism leading to politically motivated attacks against civilians. The failures to see such continuities between the invisible or private violence against women, and its links to close links to domestic terrorism, is representative of an ongoing failure to take women's security seriously (McCulloch, et al., 2019). The oftentimes invisible misogyny of groups such as Incels should be taken as a stern reminder that misogyny is indeed prevalent within contemporary society, as the Black Pill's desire for a return to the "golden age" (Bael et al., 2019, p.13) necessarily supports rolling back hard-fought feminist gains, and promotes political violence against women.

However, labelling the entire group of Incels as an "emerging domestic terrorism threat" (Texas Department of Public Safety, 2020, p. 3) is problematic, as studies in social psychology reveal that within the contemporary political and security environment the use of labels such as 'terrorist' have become close to the ultimate out-group, in that they are immediately cast as threatening or dangerous (Bael, Sterck, Slingeneyer, \& Lits, 2019). The mass media and online watch-groups such as Incel Tears play a role in prompting such problematic labels with the growing base of media articles, opinion columns and the hive of social media activity discussing the perceived threat that Incels pose. Discussing Islamic terrorism, Wardlaw (1989) explained, "institutions and roles [related to terrorism] become reified," (p. 5) establishing 
moral judgements and expectations. As Bael et al., (2019b) highlighted, evoking such narratives of in/out-group categorisation bestows on the labelled individual (or group) extremely negative characteristics, triggers negative feelings and elicits hard-line policy choices against those associated with the group. Furthermore, the labelling of 'terrorist' or 'terror group' opens individuals and groups up to strategies of exclusion, monitoring, revised policing techniques, far reaching intelligence and surveillance measures and policies of early prevention (Mythen, 2014; Schmid, 2013). The labelling and monitoring of 'terrorist groups' (and the large amounts of funding such initiatives receive), runs contrary to policies that defund domestic violence prevention strategies, rape shelters, progressive gender education and other anti-violence services (Pain, 2014). As the realities of everyday violence and global terrorism are intimately connected, a more empathetic response must address the people suffering the effects of everyday terrorism. Policies of 'othering' and exclusion for Incels, I suggest, are particularly detrimental, as it appears that isolation contributes to their extremism in the first place. The silencing and exclusion of Incels also removes any potential for nuanced political discourse with individuals from such groups, which may work to reveal to Incels their problematic worldview that is based in hateful and sentimental discourse, and surface-level readings of scientific information. Rather, I argue that we should treat the ideology of the Black Pill that is rooted in a militant form of misogyny as a threat, and work to combat such discourse and beliefs at the structural levels to prevent such potential for radicalisation.

\section{Conclusion:}

Throughout this qualitative exploration of the Incel subculture, I aimed to provide a counterpoint to the individualising narratives that much Incel-focused research has taken. My data revealed that for Incels, the acts of individuals can be observed in reference to the wider structures of masculinity and economic anxiety (Chapter Four), the cultural era of postfeminism (Chapter Five), and the ideology of the dehumanising, radicalising discourse of the Black Pill (Chapter Six). These aspects interact with individual life histories of Incels, who in varying degrees of belief accept the 'truth' of the Black Pill; a truth that requires believers to blame, and therefore punish, women for their positions within society. This finding directly highlights the relationship between gendered and structural violence, and stochastic terrorism rooted in militant misogyny.

In exploring the Incel subculture through examining the discourse, memes and other cultural material forged and (re)produced through Incel forums, I identified that through the Black Pill's 
shared sense of a mythology of victimisation, Incels unwaveringly support violence against out-groups. Despite Incels (and other manosphere groups) absence of a formal structure or overarching parent organisation, the communication of the 'truth' of the Black Pill is a key facet of how it radicalises users to commit violence. The nature of such attacks are seemingly random, sporadic and individually unpredictable. As Munn (2019) has conceptualised, similar violence perpetrated by individuals connected to the Alt-Right can be viewed as 'stochastic terrorism', which allows members to choose their own targets, locations and specific motivations.

It may be easy to believe that Incels are an extreme fringe group that do not pose a threat to national or international security. However, Incels represent just one part of a spectrum of extremist groups spanning a vast range of political ideologies, united by militant misogyny. These groups range from white supremacists and neo-Nazis, to the radical Islam of groups such as ISIS. Incels' Black Pill ideology is just one aspect of a violent ideological masculinity that underpins these extremist worldviews. Such ideologies, which are based on ideas of domination through fear and violent acts, should be considered a form of terrorism (Zimmerman, et al., 2019). However, I have argued that such labels are also problematic as they create political legitimacy to engage in heavy-handed policing and surveillance of such individuals and groups. It is vital that future research further explores the resurgence to traditional, patriarchal cultures, as it is a core feature that unites violent extremist beliefs.

This thesis unpacked and provided empirical evidence to support the insights offered by Ging's (2017, 2019) research on the interconnections between the constellation of 'men's rights' groups that compose the manosphere. It built specifically on Ging's (2019) understanding of Incels' negotiation with contemporary digital gender politics and detailed the subculture's relationship with hegemonic masculinity and a postfeminist society. Building on Bael et al's (2019) discussion of the Black Pill, I detailed the core facets of the worldview, and highlighted specific ways in which Incel ideology works to radicalise new members. Throughout the case study of Incels, Powell et al's (2018) theoretical framework of 'digital society' was useful in highlighting that the creation of hatred and violence transcends the false distinction of the internet and the terrestrial world. The close examination of Incels reveals that both online and offline features of society contribute to the (re)production of hatred, and that violence is ubiquitous - not merely confined to the offline sphere. 
Now that I have peeled back the layers of the Incel subculture, it is vital that future research attempts to dismantle the conceptual structures at the intersections of dominant notions of masculinity, privilege and entitlement, patriarchy, racism and xenophobia, and the acceptance of everyday forms of violence against women. Furthermore, future research that engages with how the collective venting of sentiment works to reify Incel and other manosphere groups' worldviews and addresses the clear sense of loneliness, alienation and mental health problems that are present within the community is vital. Finally, it is of utmost importance that researchers examine the specific techniques of online cultures and ideologies (such as Incels, Alt-Right, and other militant misogynists) that foster young men to commit a raft of violent actions that range from the 'invisible', everyday forms of violence to the extreme violent events displayed by 'lone wolves'. 


\section{Appendix}

\section{Incel Terminology Dictionary}

Alpha (Male) - The opposite to a beta male. Takes on risks and confrontation. Confident and a leader.

Ascend - When an Incel moves on to get laid or enter into a relationship.

AWALT - All Women Are Like That. All Women are Literal Trash. All women are lying thots. Used to generalise about women in negative ways. Erases specificity, dehumanizing.

Based - When someone is concerned about spreading the truth, but also unconcerned about any potential reaction.

Becky - an average girl, opposite to Stacy.

Beta (Male) - opposite to alpha; lacking charisma and shy away from confrontation.

Originated from biology, misapplied to humans, predominantly in MRA and PUA.

Betabuxx - Describes an Incel who manages to find a partner. Refers to an imagined time at which women suddenly start to lose SMV (sexual market value) and decide to settle for a man who can support them (usually financially).

In addition, betabuxxing involves financially supporting a woman whilst she finds sexual fulfilment with Chad elsewhere, cucking the beta man. Incels typically dislike men who betabuxx, dismissing the idea of attracting a women later in life because of the fear of being treated this way.

Black Pill - Nihilistic cousin of the Red Pill (The idea being that men recognise that the world is unfair and stacked against them in favour of women. From there, men can start to game the system and become 'alpha' males, going to the gym, treating women poorly etc.) The Blackpill rejects this, saying that there can be no personal solutions to system oppression, and that the world was, is and always will be stacked against men who are 'genetically inferior', and that women are inherently wired to prefer men with particular kinds of facial features, bone structures and body types.

The Blackpill is problematic insofar as it also means that those who subscribe to it are either doomed to a life of misery or have to take part in violent retribution to prevent this. This core concept highlights how Incels discourse obsessively leans backwards and forwards in discussions of mass-murder or suicide.

Blue Pill - refers to someone who is blissfully and wilfully ignorant of societal disadvantage of men.

BTFO - "Blown The Fuck Out" meaning a certain comment is demoralizing or demotivating to a group/individual. It can be used to state that someone lost an argument.

Blackops2cel - Also known as, St.BlackOps2Cel is the archetype of the Incel community. Emblematic of what an Incel looks like as per their community have expressed. 
Braincels $-\mathrm{r} / \mathrm{braincels}$, the main Incel subreddit since $\mathrm{r} /$ incels was removed from Reddit in Nov 2017 for violating site-wide rules. Braincels posters tend to tow the Reddit line of adhering to the sites anti-harassment and incitement to violence rules.

Chad - The anti-Incel. A Chad is a man who is sexually successful, who is charismatic, handsome and clever (although, Chad is often portrayed as unintelligent or Jock-like). Incels who have odd relationship with Chad, simultaneously loathing and worshipping him. Their theory is that even women who eventually marry 'betas' will still desire Chad, and will almost invariably cheat on and 'cuck' their husband with Chad. The undeserving victors in the genetic lottery, with everything coming to them easily.

Common Chad monikers include Chad, Chadrone (for a mixed-race Chad; also see: Tyrone), Chang (East Asian Chad), Chadpreet (Indian Chad), Chaddam (Arabic).

Chadlet - A manlet (short) Chad.

Chadlite - Someone who is almost a Chad, but not quite. Ranked above a normie on the social hierarchy.

ClownWorld - Clown World or Heil Honkler is an alt-right propaganda meme. The "Honkler" image first appeared on 4chan in February 2019 Clown World and Honkler are part of the alt-right effort to obfuscate racism and hate with seemingly innocuous memes. Its users claim it simply means that the (Western) world is so "crazy" in its embrace of social justice politics (read: not racist) that the only people who could conceivably be running it are Jews clowns, hence "clown world." In reality, countries without closed borders and which don't allow for the genocide of minorities are "clown countries" because they do not embrace the policies of the Third Reich.

Cock Carousel - Term used to describe what Incels think that women do before eventually settling down. "Riding the cock carousel" is a common phrase on Incel forums. The idea is that women have sex with as many 'high quality' men as possible, moving from one to another without thought. Women are usually 16-25 while riding the carousel (in Uni).

Cope - According to the Blackpill there can be no personal solutions to structural problems, so no amount of working out, diet, showering and self-improvement can improve the status of an Incel. Anything an Incel does is necessarily a 'cope' - a temporary way of making themselves feel better in the situation. This mind-set becomes self-perpetuating, as it leads to thinking that any path to self-improvement is ultimately doomed, thus no need to try. Can be used to indicate disagreement with a post.

Cuck - A man whose wife/girlfriend is having sex with another man. There are racial overtones, with the word often referring specifically to women cheating with black men. There is also BDSM-like connotations, as the man supposedly gets off on the humiliation.

Currycel - A man who is involuntarily celibate because he is of Indian or similar descent.

ER or 'going ER' - Elliot Rodger, the man who shot and killed multiple people in Isla Vista California in 2014. A martyr on Incel forums, 'going ER' refers to an Incel who is deemed likely to follow a similar pattern of behaviour and commit mass-murder.

Escourtcel - an Incel who uses the services of a sex-worker to get laid. 
Fakecel - a person who claims to be an Incel, but is not. Incel gatekeeping.

Femcels - Female Incels, which "don't exist" according to many comment threads on $\mathrm{r} /$ Braincels and Incels.co. Reddit.com/r/Femcels is a community in which involuntary celibate women can discuss their involuntary celibacy, however, it is emphasised by male Incels that women cannot be Incels.

Foid/Femoid - Abbreviated form of "female humanoid", used to dehumanize females. Sometimes refers to women as 'It' to further dehumanize.

Fuckzoned - when a femcel is used in a relationship for nothing more than sex.

-fuel - Motivation for something e.g., Lifefuel.

FWHR - Face/width height ratio.

GTFIH - "Get the Fuck In Here" i.e. read this post.

Gymcel - an Incel who works out.

High IQ - Indicates agreement with a previously stated point.

Hole - Refers to any female.

Hunter Eyes - The squinty, hooded shape of a particular set of eyes. Coupled with an aesthetic shae - when the outer corner is higher than the inner like so, also known as positive canthal tilt (a positive trait).

Hypergamy - A term borrowed from biology used to described the phenomenon of females mating with males of a higher status. MRAs and Incels apply it to humans, arguing that women attempt to find men who are higher status than they are. This gives birth to what they call the 80:20 rule: that the top 20\% of men are being competed for by the top $80 \%$ of women, and the bottom $80 \%$ of men are competing for the bottom $20 \%$ of women.

Incel - Involuntary Celibate.

Inhibition / Inhib - Can be described as low or high. Low inhib guys don't care what others think of them and are thus perceived as confident/alpha. High inhib guys think about what others think about them and let that limit themselves by being passive.

Inkwell - sarcastic term for Incel, usually in reference to news media / other forums poking fun at Incels.

IT - IncelTears, forum on reddit which is inherently anti-Incel.

JBW - Just Be White - used to imply that just being white can resolve all Incel problems.

JB - Jail Bait (underage girl who is sexually desirable, yet difficult to determine if she is old enough).

Jestering/JesterMAXING - a professional joker or 'fool' at a medieval court, typically wearing a cap with bells on it and carrying a mock sceptre; used in Incel terms in a similar way as a 'cuck' or 'beta', but used when someone is found particularly hilarious.

KEK - Different form of 'Lol' originated in World of Warcraft. 
KHHV - Kissless, Handholdless, Hugless virgin.

KV - Kissless Virgin.

Landwhale - an overweight woman.

Lanklet - Tall, skinny, and therefore unattractive man.

LARP / LARPer / LARPing - a type of role-playing game in which participants physically act out scenarios, typically using costumes and props. In Incel terms it often is used to infer someone is acting/pretending to be an Incel

LDAR - Lay Down and Rot. Meaning to give up on life.

Looksmatch - Looksmatches are of similar attractiveness levels, both a 6/10 or such. Often connoted with women who try to strive beyond their looksmatch i.e. go for an 8 when she's a 6.

Low IQ - indicates disagreement with previously stated point.

Lowinhibmaxx - to get a low inhibition, to be someone with a low inhibition. Presumably an effort to increase testosterone levels to become confident when approaching the other gender.

LTR - Long Term Relationship.

Manlet - short guy, usually quick stocky.

-maxx/-maxxing/-max - efforts to improve an aspect of one's life to the fullest. Maximise it.

Mentalcel - someone who is an incel because of mental illness-related reasons.

MGTOW - Men Going Their Own Way, a forum and community dedicated to the belief that women are toxic and detrimental to society. These men sometimes cross-pollinate with Incel beliefs but not always.

Mog - to overshadow, especially in personal aesthetic attributes, often supported with a facial feature.

Mogger - generally used as a racist term, when a black man 'mogs' another race.

NEET - Not in Education, Employment or Training - widespread, not Incel-specific, but is common in a lot of discussion about/with them and between themselves.

Nice Guy - a guy who acts nice to women in an attempt to get laid, proceeds to get angry and complain when it doesn't work.

Noodlewhore - an Asian woman.

Normie - anyone besides Incel or Chad/Stacy or Incels.

NPC - Non-Playing Character, someone who repeats group ideas with no critical thinking. No original thought.

NT - neurotypical i.e. not on the autism spectrum. Not unique to Incels but often used. 
Numale - Nu-males are men lacking self-respect who are completely devoid of any masculinity and will jump at any moment to defend women online for feminist brownie points while falsely believing that in return, they will receive sexual favours.

Orbiter / beta-orbiter - a guy that wants to sleep with his female friend, he orbits her (hangs out with her in a needy way) in the hopes of getting sex someday.

Personality - (r/Braincel automoderator) "Personality isn't really important to most women. They often just euphemize personality for good physical looks and social status.”

Personality Detector - used to mock women who stick with abusive significant others or women who enter into relationships with a negative background. Incels are pointing out the failure of some women to detect weird feelings about those who have negative/malicious intentions.

-pill - can be used as a suffix for any Incel 'realisation' e.g. racepill; agepill etc.

-pilled - the act of accepting one of the 'pills' as the truth.

Red pill - The idea being that men recognise that the world is unfair and stacked against them in favour of women. From there, men can start to game the system and become 'alpha' males, going to the gym, treating women poorly.

Reee - commonly referred to now as an 'autistic screeching meme', this is an onomatopoeic expression of intense rage or frustration, typically is associated with the Angry Pepe character. Used throughout the manosphere.

Rice - relating to someone from Asia.

Roastie - Used to describe a women who has had sex with more than one partner. Combines both body and slut-shaming practices.

Rope - to suggest or commit suicide by hanging.

SMV - sexual market value.

Snowbunny - a white woman who likes or is in a relationship with a black man.

Soy - Basing off studies, which suggest, but do not prove, that soy lowers testosterone, Incels use this to suggest that someone is low in testosterone.

Stacy - a hot, popular woman. The archetype of a desirable woman.

Sui - Suicide.

Tallfag - a tall man.

TFL - True Forced Loneliness - similar to Volcel, may indicate a disability or other ailment that forces the Incel to be alone.

Truecel - the opposite of volcel and fakecel, used to indicate that there is no hope for them.

Turbo - an extreme case.

Tyrone - used to denote a black man who is a Chad equivalent, though usually also with racist and classist overtones. Tyrone might cuck you, but Stacy will ultimately leave Tyrone 
for Chad because Chad is white. Incels believe that women will always prefer white men because of the power and status they hold.

Volcel - used to indicate that the reason is celibate is a voluntary decision, e.g. "Fatcels are volcels."

Wage Cuck - a wagecuck is a someone who works a traditional job (a wageslave) while not being in a relationship and not having a potential for a relationship.

The Wall - after riding the cock carousel, this is the idea that a woman is used up and worthless. Specifically relates to age and the loss of youthful appearance.

White Knight - a male who takes things out of context to adamantly defend women's rights. White Knights tend to make an issue out of something when there is no issue present. Exhibits Nice Guy behaviour when doing so.

Wristcel - A man who is Incel because of the circumference of his wrists. Again, because this is a relatively immutable quality, much like other aspects of bone structure, it is seen as an unchangeable marker of genetic inferiority, and therefore something that will condemn Incels to sexual celibacy. 


\section{References}

Allely, C. S., \& Faccini, L. (2017). "Path to intended violence" model to understand mass violence in the case of Elliot Rodger. Aggression and violent behavior, 37, 201-209.

DOI:10.1016/j.avb.2017.09.005

Anderson, E. (2005). Orthodox and inclusive masculinity: Competing masculinities among heterosexual men in a feminized terrain. Sociological perspectives, 48(3), 337-355. DOI:10.1525/sop.2005.48.3.337

Awan, I. (2017). Cyber-extremism: Isis and the power of social media. Society, 54(2), 138-149. DOI: $10.1007 / \mathrm{s} 12115-017-0114-0$

Baele, S., J, Brace, L., \& Coan, T., G. (2019). From "Incel” to "Saint": Analyzing the violent worldview behind the 2018 Toronto attack. Terrorism and Political Violence, DOI:10.1080/09546553.2019.1638256.

Bael, S., J. Sterck, O., C. Slingeneyer, T. \& Lits, G., P. (2019). What Does the "Terrorist” Label Really Do? Measuring and Explaining the Effects of the "Terrorist" and "Islamist" Categories. Studies in Conflict \& Terrorism, 42(5), 520-540, DOI:10.1080/1057610X.2017.1393902.

Banet-Weiser, S., \& Miltner, K. M. (2016). \#MasculinitySoFragile: culture, structure, and networked misogyny. Feminist Media Studies, 16(1), 171-174. DOI:10.1080/14680777.2016.1120490

Barlett, C. P., Vowels, C. L., \& Saucier, D. A. (2008). Meta-analyses of the effects of media images on men's body-image concerns. Journal of social and clinical psychology, 27(3), 279-310. DOI:10.1521/jscp.2008.27.3.279 
Bassett, E. H., \& O'riordan, K. (2002). Ethics of Internet research: Contesting the human subjects research model. Ethics and Information Technology, 4(3), 233-247. DOI: 10.1023/A:1021319125207

Bauman, Z. 2000. Modernity and the Holocaust. Ithaca, N.Y.: Cornell University Press.

Baumeister, R. F., \& Vohs, K. D. (2004). Sexual economics: Sex as female resource for social exchange in heterosexual interactions. Personality and Social Psychology Review, 8(4), 339363. DOI: $10.1207 / \mathrm{s} 15327957$ pspr0804_2

Baym, N. K. (2015). Personal connections in the digital age. John Wiley \& Sons.

Bharti, B. (2019). US Army warns of possible incel shootings at joker film screening, Retrieved from https://nationalpost.com/news/world/u-s-army-warns-of-possible-incel-shootings-at-jokerfilm-screenings

Benach, J., Amable, M., Muntaner, C., \& Benavides, F, G. (2002). The consequences of flexible work for health: are we looking at the right place? Journal of Epidemiology and Health. 56(6). DOI: 10.1136/jech.56.6.405

Berger, P. L. (1966). The social construction of reality. New York: Anchor Books.

Berger, R. (2015). Now I see it, now I don't: Researcher's position and reflexivity in qualitative research. Qualitative research, 15(2), 219-234. DOI:10.1177/1468794112468475

Bergstrom, K. (2016). An Unwelcome Intrusion? Player Responses to Survey Research Recruitment on the World of Warcraft Forums. Loading... The Journal of the Canadian Game Studies Association, 10(16).

Beuchamp, Z. Our Incel Problem retrieved from https://www.vox.com/thehighlight/2019/4/16/18287446/incel-definition-reddit 
Biondi, K., \& Curtis, J. (2018). From Structural to Stochastic Violence. EDITORA DA UEMA, 46.

Bird, S. R. (1996). Welcome to the men's club: Homosociality and the maintenance of hegemonic masculinity. Gender \& society, 10(2), 120-132. DOI: 10.1177/089124396010002002

Bourgois, P. (1995). In Search of Respect. Cambridge: Cambridge University Press.

Boyd, S. B. (2004). Demonizing mothers: Fathers' rights discourses in child custody law reform processes. Journal of the Motherhood Initiative for Research and Community Involvement, 6(1).

Brantingham, P. (2011). Crime and place: rapidly evolving research methods in the 21st century. Cityscape, 13(3), 199-203.

Bratich, J., \& Banet-Weiser, S. (2019). From pick-up artists to incels: con (fidence) games, networked misogyny, and the failure of neoliberalism. International Journal of Communication, 13. DOI:1932-8036/2019FEA0002

Braun, V., \& Clarke, V. (2006). Using thematic analysis in psychology. Qualitative research in psychology, 3(2), 77-101. DOI:10.1191/147808876qp063oa

Bridges, T., \& Pascoe, C. J. (2014). Hybrid masculinities: New directions in the sociology of men and masculinities. Sociology Compass, 8(3), 246-258. DOI:10.1111/soc4.12134

Briffault, R. (1931). The mothers: The matriarchal theory of social origins. New York: The MacMillan Company.

Brown, S. (2006). The criminology of hybrids: Rethinking crime and law in technosocial networks. Theoretical Criminology, 10(2), 223-244. DOI:10.1177/1362480606063140

Bryant, A., \& Charmaz, K. (Eds.). (2007). The Sage handbook of grounded theory. Sage. 
Carpenter, L. (2005). Virginity loss: An intimate portrait of first sexual experiences. New York: New York University Press.

Casti, T. (2014). Women play video games. Can we cut the sexist crap now? Huffington Post. Retrieved from http://www.huffingtonpost.com/2014/04/24/female-gamers_n_5207137.html

Ceruzzi, P. E., \& Paul, E. (2003). A history of modern computing. MIT press.

Charles, M., \& Grusky, D. B. (2007). Egalitarianism and gender inequality. In Grusky, D. \& Szelényi, S. (eds) The inequality reader: Contemporary and foundational readings in race, class, and gender, 327-342.

Chan, J., \& Bennett Moses, L. (2016). Is big data challenging criminology?. Theoretical criminology, 20(1), 21-39. DOI: 10.1177/1362480615586614

Charmaz, K. (2000). Constructivist and objectivist grounded theory. In N. K. Denzin \& Y. Lincoln (Eds.), Handbook of qualitative research (2nd ed., 509-535). Thousand Oaks, CA: Sage.

Charmaz, K. (2014). Constructing grounded theory (2nd ed.). Thousand Oaks, CA: Sage.

Chess, S., \& Shaw, A. (2015). A conspiracy of fishes, or, how we learned to stop worrying about\# GamerGate and embrace hegemonic masculinity. Journal of Broadcasting \& Electronic Media, 59(1), 208-220. DOI:10.1080/08838151.2014.999917

Clover, J. 2019. Four notes on stochastic terrorism. Retrieved from https://popula.com/2019/04/03/four-notes-on-stochastic-terrorism/, accessed 03 February, 2020.

Cohen, S. (1993). Human rights and crimes of the state: the culture of denial. Australian \& New Zealand Journal of Criminology, 26(2), 97-115. 
Connell, R. 1987. Gender and Power: Society, the Person, and Sexual Politics. Stanford University Press.

Connell, R. W., \& Messerschmidt, J. W. (2005). Hegemonic masculinity: Rethinking the concept. Gender \& society, 19(6), 829-859. DOI: 10.1177/0891243205278639

Coston, B. M., \& Kimmel, M. (2012). Seeing privilege where it isn't: Marginalized masculinities and the intersectionality of privilege. Journal of Social Issues, 68(1), 97-111. DOI: $10.1111 / \mathrm{j} .1540-4560.2011 .01738 . x$

Croall, H. (1992). White collar crime (p. 64). Buckingham: Open University Press.

Crosset, V., Tanner, S., \& Campana, A. (2019). Researching far right groups on Twitter: Methodological challenges 2.0. New Media \& Society, 21(4), 939-961. DOI: $10.1177 / 1461444818817306$

Crotty, M. (1998). The foundations of social research: Meaning and perspective in the research process. St Leonards, NSW: Allen \& Unwin.

Daly, K., \& Chesney-Lind, M. (1988). Feminism and criminology. Justice quarterly, 5(4), 497-538. DOI:10.1080/07418828800089871

Danner, C., \& Raymond, A, K. (2019) Everything we know about the Dayton mass-shooting. Retrieved from http://nymag.com/intelligencer/2019/08/everything-we-know-about-thedayton-mass-shooting.html

Décary-Hétu, D., \& Dupont, B. (2012). The social network of hackers. Global Crime, 13(3), 160175. DOI:10.1080/17440572.2012.702523

Demetriou, D. Z. (2001). Connell's concept of hegemonic masculinity: A critique. Theory and Society, 30(3), 337-361. 
Dickson-Swift, V., James, E. L., Kippen, S., \& Liamputtong, P. (2007). Doing sensitive research: what challenges do qualitative researchers face? Qualitative research, 7(3), 327-353. DOI: $10.1177 / 1468794107078515$

Dragiewicz, M. (2008). Patriarchy reasserted: Fathers' rights and anti-VAWA activism. Feminist Criminology, 3(2), 121-144. DOI: 10.1177/1557085108316731

Dragiewicz, M. (2011). Equality with a vengeance: Men's rights groups, battered women, and antifeminist backlash. Northeastern University Press.

Douthat, R. (2018). The Redistribution of Sex. Retrieved from https://www.nytimes.com/2018/05/02/opinion/incels-sex-robots-redistribution.html

Dwyer, S. C., \& Buckle, J. L. (2009). The space between: On being an insider-outsider in qualitative research. International journal of qualitative methods, 8(1), 54-63.

DOI:/10.1177/160940690900800105

Eckstein, J. J. (2011). Reasons for staying in intimately violent relationships: Comparisons of men and women and messages communicated to self and others. Journal of family violence, 26(1), 21-30. DOI: 10.1007/s10896-010-9338-0

Faccini, L., \& Allely, C. S. (2017). Rare instances of individuals with autism supporting or engaging in terrorism. Journal of Intellectual Disabilities and Offending Behaviour, 8(2) 70-82. DOI:10.1108/JIDOB-11-2016-0022

Faludi, S. (1992). Backlash: The Undeclared War Against American Women, $2^{\text {nd }}$ ed (New York: Doubleday).

Flood, M. (2008). Men, sex, and homosociality: How bonds between men shape their sexual relations with women. Men and Masculinities, 10(3), 339-359.

DOI:10.1177/1097184X06287761 
Follman, M. (2019). Armed and Misogynist: How Toxic Masculinity Fuels Mass Shootings. Retrieved from https://www.motherjones.com/crime-justice/2019/06/domestic-violencemisogyny-incels-mass-shootings/

Foucault, M. (1978). The History of Sexuality. New York: Pantheon Books.

France, A. (2008). Being to Becoming: The Importance of Tackling Youth Poverty in Transitions to Adulthood. Social Policy and Society, 7(5) 495-505. DOI: 10.1017/S1474746408004454

Friedman, J. (2013). A Look Inside the 'Men's Rights' Movement That Helped Fuel California Alleged Killer Elliot Rodger. Retrieved from https://prospect.org/power/look-inside-men-srights-movement-helped-fuel-california-alleged-killer-elliot-rodger/

Friedrichs, D. O. (2009). Trusted criminals: White collar crime in contemporary society. Cengage Learning.

Fuchs, C. (2014). Digital prosumption labour on social media in the context of the capitalist regime of time. Time \& Society, 23(1), 97-123. DOI: 10.1177/0961463X13502117

Furlong, A. (2006). Not a Very NEET Solution: Representing Problematic Labour Market Solutions among Early School-Leavers. Work, Employment and Society, 20(6) 553-569 DOI: $10.1177 / 0950017006067001$

Galasso, N. (2019). Confronting Far-Right "Masculinities”: Implications for NGOs, Turkish Policy Quarterly. 18(3), 101-108.

Gergen, K. J. (1999). An invitation to social construction. Sage.

Glaser, B. G., \& Strauss, A. L. (1967). The discovery of grounded theory: Strategies for qualitative research. Chicago, IL: Aldine. 
Gill, R. (2007). Postfeminist media culture: Elements of a sensibility. European Journal of Cultural Studies 10(2), 147-166. DOI: 10.1177/1367549407075898

Gill, R. (2017). The affective, cultural and psychic life of postfeminism: A postfeminist sensibility 10 years on. European Journal of Cultural Studies, 20(6), 606-626. DOI: $10.1177 / 1367549417733003$

Ging, D. (2017). Alphas, betas, and incels: Theorizing the masculinities of the manosphere. Men and Masculinities, 22(4), 638-657. DOI: 10.1177/1097184X17706401

Ging, D. (2019). Bros v. Hos: Postfeminism, Anti-feminism and the Toxic Turn in Digital Gender Politics. In Ging D, \& Siapera, E. (Eds) Gender Hate Online: Understanding the New Antifeminism (pp. 45-67). Palgrave Macmillan, Cham.

Ging, D., \& Siapera, E. (2018). Special issue on online misogyny. Feminist Media Studies, 18(4), 515-524, DOI: 10.1080/14680777.2018.1447345.

Gotell, L., \& Dutton, E. (2016). Sexual violence in the 'manosphere': Antifeminist men's rights discourses on rape. International Journal for Crime, Justice and Social Democracy, 5(2), 65. DOI: $10.5204 /$ ijcjsd.v5i2.310

Grabosky, P. N. (2001). Virtual criminality: Old wine in new bottles?. Social \& Legal Studies, 10(2), 243-249.

Green, A. (2019). Cucks, Fags and Useful Idiots: The Othering of Dissenting White Masculinities Online, In Harmer, E., \& Lumsden, K. Online Othering pp. 65-96. Palgrave Macmillan, Cham.

Greig, A. (2019). Masculinities and the Far-Right: Implications for Oxfam's Work on Gender Justice," Oxfam America, retrieved from https://www.oxfamamerica.org/explore/researchpublications/masculinities-and-the-far-right 
Hankes, K., \& Amend, A. (2018). The Alt-Right is Killing People. Retrieved from https://www.splcenter.org/20180205/alt-right-killing-people

Hanson, R. (2018) Two Types of Envy. Retrieved from http://www.overcomingbias.com/2018/04/two-types-of-envy.html

Haraway, D. (1988). Situated knowledges: The science question in feminism and the privilege of partial perspective. Feminist studies, 14(3), 575-599. DOI: 10.2307/3178066

Harmer, E., \& Lumsden, K. (2019). Online Othering: An Introduction. In Harmer, E., \& Lumsden, K (eds) Online Othering (pp. 1-33). Palgrave Macmillan, Cham.

Harrington, C. (2003). Phallic Masculinity as Chimera. The Independent Cultural Journal "I” 27, 117.

Hawkins, C. 2019. The threat from far-right extremism: Rising, less predictable and hard to determine. Retrieved from https://ihsmarkit.com/research-analysis/the-threat-from-far-rightextremism-rising.html, accessed 03 February, 2020.

Henry, N., \& Powell, A. (2015). Embodied harms: Gender, shame, and technology-facilitated sexual violence. Violence against women, 21(6), 758-779. DOI: 10.1177/1077801215576581

Henry, N., \& Powell, A. (2016). Sexual violence in the digital age: The scope and limits of criminal law. Social \& Legal Studies, 25(4), 397-418. DOI: 10.1177/0964663915624273

Hergovich, P., \& Ortega, J. (2018). The strength of Absent Ties: Social Integration via Online Dating. SSRN. DOI: 10.2139/ssrn.3044766

Hesse-Biber, S. N., \& Leavy, P. (2011). The practice of qualitative research. Thousand Oaks, CA: Sage. 
Hesse-Biber, S, N. (2017). The Practive of Qualitative Research. $3^{\text {rd }}$ edition. Thousand Oaks, CA: Sage.

Hunter, M, A., \& Robinson, Z, F. (2016). The Sociology of Urban Black America. Annual Review of Sociology. 42, 385-405. DOI: 10.1146/annurev-soc-081715-074356

Jaki, S., De Smedt, T., Gwóźdź, M., Panchal, R., Rossa, A., \& De Pauw, G. (2019). Online hatred of women in the Incels. me forum: Linguistic analysis and automatic detection. Journal of Language Aggression and Conflict, 7(2), 240-268. DOI: 10.1075/jlac.00026.jak

Jane, E. A. (2014). 'Back to the kitchen, cunt': Speaking the unspeakable about online misogyny. Continuum, 28(4), 558-570. DOI: 10.1080/10304312.2014.924479

Jane, E. A. (2016). Online misogyny and feminist digilantism. Continuum, 30(3), 284-297. DOI: $10.1080 / 10304312.2016 .1166560$

Jones, C., Trott, V., \& Wright, S. (2019). Sluts and soyboys: MGTOW and the production of misogynistic online harassment. New Media \& Society, DOI: 1461444819887141.

Johnston, M., \& True, J. (2019). Misogyny \& violent extremism: Implications for preventing violent extremism. Policy Brief. Monash University.

Jordan, A. (2016). Conceptualizing Backlash:(UK) Men's Rights Groups, Anti-Feminism, and Postfeminism. Canadian Journal of Women and the Law, 28(1), 18-44. DOI: 10.3138/cjwl.28.1.18

Kanuha, V. K. (2000). "Being" native versus "going native": Conducting social work research as an insider. Social work, 45(5), 439-447. DOI: 10.1093/sw/45.5.439

Kelan E (2009) Performing Gender at Work. New York: Palgrave Macmillan 
Kenway, J., \& Kraack, A. (2004). Reordering work and destabilizing masculinity. In Dolby, N., Dimitriadis, G., \& Willis, P. (eds) Learning to labor in new times, (pp 95-109). New York: Routledge Falmer.

Kimmel, M. (2010). Misframing Men: The politics of contemporary masculinities. New Brunswick: Rutgers University Press.

Kimmel, M. (2015). Angry White Men: American Masculinity at the End of an Era. New York: Nation Books.

Kimmel, M. (2017). Manhood in America. New York, NY: Oxford University Press.

Kimmel, M. S., \& Mahler, M. (2003). Adolescent masculinity, homophobia, and violence: Random school shootings, 1982-2001. American behavioral scientist, 46(10), 1439-1458. DOI: $10.1177 / 0002764203046010010$

Kirkwood, C. (1993). Leaving abusive partners. Newbury Park: Sage.

KhosraviNik, M., \& Esposito, E. (2018). Online hate, digital discourse and critique: Exploring digitally-mediated discursive practices of gender-based hostility. Lodz Papers in Pragmatics, 14(1), 45-68. DOI: 10.1515/lpp-2018-0003

Kupers, T. A. (2005). Toxic masculinity as a barrier to mental health treatment in prison. Journal of clinical psychology, 61(6), 713-724. DOI: 10.1002/jclp.20105

Levchak, C. C. (2018). Microaggressions and modern racism: Endurance and evolution. Springer.

Lewis, R., Rowe, M., \& Wiper, C. (2019). Online/Offline Continuities: Exploring Misogyny and Hate in Online Abuse of Feminists. In Harmer, E., \& Lumsden, K (Eds.) Online Othering (pp. 121-143). Palgrave Macmillan, Cham. 
Lilly, M. (2016). 'The World Is Not a Safe Place for Men': The Representational Politics of the Manosphere. Political Science, Université d'Ottawa/University of Ottawa.

Light, R., \& Kirk, D. (2000). High school rugby, the body and the reproduction of hegemonic masculinity. Sport, education and society, 5(2), 163-176. DOI: 10.1080/713696032

Lokke, G. (2019). Cuckolds, cucks, and their transgressions. Porn Studies, 6(2), 212-227. DOI: $10.1080 / 23268743.2018 .1555053$

Louie, M. (2018). The Incel (Involuntary Celibacy) Problem: How are Incels different from others who struggle with romance. Retrieved from https://www.psychologytoday.com/us/blog/minority-report/201804/the-incel-involuntarycelibacy-problem

Lupton, D. (2015). Digital Sociology. New York: Routledge.

Maddison, S. (1999). Private men, public anger: The men's rights movement in Australia. Journal of Interdisciplinary Gender Studies: JIGS, 4(2), 39. DOI: 803980536786452

Manne, K. (2017). Down girl: The logic of misogyny. Oxford University Press.

Markham, A., \& Buchanan, E. (2002). Ethical decision-making and internet research: recommendations from the AoIR ethics working committee (Version 2.0). Retrieved from http://www. aoir. org/reports/ethics.pdf.

Maynard, M. (1994). Methods, practice and epistemology: The debate about feminism and research. In M. Maynard \& J. Purvis (Eds.), Researching women's lives from a feminist perspective (pp. 1-26). Abingdon: Routledge.

McCulloch, J. \& Wilson, D. (2015). Precrime: Pre-emption, precaution and the future. New York: Routledge. 
McCulloch, J., Walklate, S., Maher, J. M., Fitz-Gibbon, K., \& McGowan, J. (2019). Lone Wolf Terrorism Through a Gendered Lens: Men Turning Violent or Violent Men Behaving Violently? Critical Criminology. 27(3), DOI: 10.1007/s10612-019-09457-5

McDowell, L. (2003). Redundant Masculinities? Employment Change and White Working Class Youth. Oxford: Blackwell

McDowell, L. (2019). Looking for work: youth masculine disadvantage and precarious employment in post-millennium England. Journal of Youth Studies. DOI: $10.1080 / 13676261.2019 .1645949$

McRobbie, A. (2009) The Aftermath of Feminism: Gender, Culture and Social Change. London: SAGE.

Megarry, J. (2014). Online incivility or sexual harassment? Conceptualising women's experiences in the digital age. Women's Studies International Forum, 47, 46-55. DOI: 10.1016/j.wsif.2014.07.012

Massanari, A. (2017). \#Gamergate and The Fappening: How Reddit's algorithm, governance, and culture support toxic technocultures. New Media \& Society, 19(3), 329-346. DOI: $10.1177 / 1461444815608807$

Marwick, A. E., \& Caplan, R. (2018). Drinking male tears: language, the manosphere, and networked harassment. Feminist Media Studies, 18(4), 543-559. DOI: 10.1080/14680777.2018.1450569

Messner, M. A. (1998) The limits of 'the male sex role': An analysis of the men's liberation and men's rights movements' discourse. Gender \& Society 12(3): 255-276. DOI: $10.1177 / 0891243298012003002$. 
Messner, M. A. 2016. Forks in the Road of Men's Gender Politics: Men's Rights vs Feminist Allies. International Journal for Crime, Justice and Social Democracy 5, 6-20.

DOI:10.5204/ijcjsd.v5i2.301

Merrit-Gray, M., \& Wuest J. (1995). Counteracting abuse and breaking free: the process of leaving revealed through women's voices. Health Care for Women International, 16. 399-412. DOI: $10.1080 / 07399339509516194$

Muncie, J. (2000). Decriminalizing criminology. In Lewis, G., Gewirtz, S., \& Clarke, J. (eds) Rethinking social policy, pp. 217-228.

Munn, L. (2019). Alt-right pipeline: Individual journeys to extremism online. First Monday, 24(6). DOI: $10.5210 / \mathrm{fm} . v 24 \mathrm{i} 6.10108$

Mythen, G. (2014). Understanding the risk society. Crime, security and justice. London: Palgrave Macmillan

Nagle, A. 2016. The New Man of 4/Chan. The Baffler, 30. Retrieved from http:// thebaffler.com/salvos/new-man-4chan-nagle.

Negra, D., and Tasker, Y. (Eds) (2014) Gendering the Recession: Media and Culture in an Age of Austerity. Durham, NC: Duke University Press.

Nicholas, L., \& Agius, C. (2018). \# Notallmen, \#Menenism, Manospheres and Unsafe Spaces: Overt and Subtle Masculinism in Anti-“PC” Discourse. In Nicholas, L., \& Agius, C. (Eds.) The Persistence of Global Masculinism (31-59). Palgrave Macmillan, Cham.

Nixon, D. (2017). Yearning to Labour? Working Class Men in Post-Industrial Britain. In Walker, C., \& Roberts, S. Masculinity, Labour and Neoliberalism: Working Class Men in International Perspective. (53-75). London: Palgrave Macmillan. 
Noaks, L., \& Wincup, E. (2004). Criminological research: Understanding qualitative methods. London: Sage.

Oksanen, A., Hawdon, J., Holkeri, E., Näsi, M., \& Räsänen, P. (2014). Exposure to online hate among young social media users. In Warehime, N, M. (ed) Sociological studies of children \& youth, 18(1), 253-273.

O’Neill, R. (2015). Whither critical masculinity studies? Notes on inclusive masculinity theory, postfeminism, and sexual politics. Men and masculinities, 18(1), 100-120. DOI: $10.1177 / 1097184 X 14553056$

Oppenheim, M. (2019). Woman who wrote book on domestic violence 'stabbed to death by her boyfriend'. Retrieved from https://www.independent.co.uk/news/world/americas/aliyah-terrymurder-stabbed-boyfriend-isaih-henderson-domestic-violence-a8998831.html.

Pain, R. (2014). Everyday terrorism: Connecting domestic violence and global terrorism. Progress in Human Geography. 38(4). 531-550. DOI: 10.1177/0309132513512231

Papacharissi, Z. (2010). A private sphere: Democracy in a digital age. Polity.

Papacharissi, Z. (2014). Affective Publics: Sentiment, Technology, and Politics. Oxford, UK: Oxford University Press

Papadamou, K., Zannettou, S., Blackburn, J., De Cristofaro, E, Stringhini, G., \& Sirivianos, M. (2020). Understanding the Incel Community on YouTube. ResearchGate, Preprint, January.

Pittaro, M. L. (2007). Cyber stalking: An analysis of online harassment and intimidation. International journal of cyber criminology, 1(2), 180-197.

Pollock, E. (2009). Researching white supremacists online: methodological concerns of researching hate 'speech'. Internet Journal of Criminology, 1-19. 
Powell, A., \& Henry, N. (2019). Technology-facilitated sexual violence victimization: Results from an online survey of Australian adults. Journal of interpersonal violence, 34(17), 3637-3665. DOI: $10.1177 / 0886260516672055$

Powell, A., Stratton, G., \& Cameron, R. (2018). Digital criminology: Crime and justice in digital society. Routledge.

Pulido, L. (2016). Flint, Environmental Racism, and Racial Capitalism. Capitalism Nature Socialism. 27(3), 1-16. DOI: 10.1080/10455752.2016.1213013

Pyke, K. D. (1996). Class-based masculinities: The interdependence of gender, class, and interpersonal power. Gender \& Society, 10(5), 527-549. DOI: 10.1177/089124396010005003

Rager, K. B. (2005). Self-care and the qualitative researcher: When collecting data can break your heart. Educational researcher, 34(4), 23-27. DOI: 10.3102/0013189X034004023

Rambukkana, N. (2019). The Politics of Gray Data: Digital Methods, Intimate Proximity, and Research Ethics for Work on the "Alt-Right”. Qualitative Inquiry, 25(3), 312-323. DOI: $10.1177 / 1077800418806601$

Reicher, S., Hopkins, N., Levine, M., \& Rath, R. (2005). Entrepreneurs of hate and entrepreneurs of solidarity: Social identity as a basis for mass communication. International review of the Red Cross, 87(860), 621-637. DOI: 10.1017/S1816383100184462

Reitman, J. (2018). US law enforcement failed to see the threat of white nationalism. Now they don't know how to stop it. The New York Times Magazine. Retrieved from nytimes.com/2018/11/03/magazine/FBI-charlottesville-white-nationalism-far-right.html.

Reyns, B. W., Henson, B., \& Fisher, B. S. (2011). Being pursued online: Applying cyberlifestyleroutine activities theory to cyberstalking victimization. Criminal justice and behavior, 38(11), 1149-1169. DOI: 10.1177/0093854811421448 
Ricciardelli, R., Clow, K. A., \& White, P. (2010). Investigating hegemonic masculinity: Portrayals of masculinity in men's lifestyle magazines. Sex Roles, 63(1-2), 64-78. DOI: 10.1007/s11199010-9764-8

Rose, P. (1985). Writing on women: Essays in a renaissance. Middletown, CT: Wesleyan University Press.

Salzinger, L. (2016). Re-marking men: Masculinity as a terrain of the neoliberal economy. Critical Historical Studies, 3(1), 1-25. DOI: 10.1086/685553

Sanders, J., Munford, R., Liebenberg, L., \& Henaghan, M. (2014). Show some emotion? Emotional dilemmas in undertaking research with vulnerable youth. Field Methods, 26(3), 239-251. DOI: $10.1177 / 1525822 X 13516842$

Segrave, M., \& Vitis, L. (Eds.). (2017). Gender, technology and violence. Taylor \& Francis.

Spitzberg, B. H., \& Hoobler, G. (2002). Cyberstalking and the technologies of interpersonal terrorism. New Media \& Society, 4(1), 71-92. DOI: 10.1177/14614440222226271

Scaptura, M. (2019). Masculinity Threat, Misogyny, and the Celebration of Violence in White Men. Master's thesis, Virginia Polytechnic Institute and State University, Virginia. Retrieved from https://vtechworks.lib.vt.edu/handle/10919/93239?show=full

Scaptura, M. \& Boyle, K. Masculinity Threat, “Incel” Traits, and Violent Fantasies Among Heterosexual Men in the United States. Feminist Criminology. DOI: $10.1177 / 1557085119896415$

Schmid, A. (1983) Political Terrorism: A Research Guide to Concepts, Theories, Databases and Literature. Amsterdam: North-Holland. 
Schmitz, R. M., \& Kazyak, E. (2016). Masculinities in cyberspace: An analysis of portrayals of manhood in men's rights activist websites. Social Sciences, 5(2), 18. DOI: $10.3390 /$ socsci5020018

Schwendinger, H., \& Schwendinger, J. (1970). Defenders of order or guardians of human rights? Issues in Criminology, 5(2), 123-157.

Stamp, G. H., \& Sabourin, T. C. (1995). Accounting for violence: an analysis of male spousal abuse narratives. Journal of Applied Communication Research, 23, 284-307. DOI: $10.1080 / 00909889509365432$

Strauss, A., \& Corbin, J. M. (1997). Grounded theory in practice. Sage.

Symons, D. (1979). The evolution of human sexuality. New York: Oxford University Press

Taub, A. (2018). On Social Media's Fringes, Growing Extremism Targets Women. Accessed, 03, February, 2020. https://www.nytimes.com/2018/05/09/world/americas/incels-torontoattack.html

Terry, G., Hayfield, N., Clarke, V., \& Braun, V. (2017). Thematic analysis. The Sage handbook of qualitative research in psychology, 17-37.

Texas Department of Public Safety. (2020) Texas Domestic Terrorism Threat Assessment, January, 2020. Retrieved from https://www.dps.texas.gov/director_staff/media_and_communications/2020/txTerrorThreatA ssessment.pdf

Tolentino, J. (2018). The Rage of the Incels. Retrieved from https://www.newyorker.com/culture/cultural-comment/the-rage-of-the-incels.

Torok, R. (2013). Developing an explanatory model for the process of online radicalisation and terrorism. Security Informatics, 2(1), 6. DOI: 10.1186/2190-8532-2-6 
United Nations. (2014). 48/104. Declaration on the Elimination of Violence against Women. United Nations General Assembly

Van Valkenburgh, S. P. (2018). Digesting the red pill: Masculinity and neoliberalism in the manosphere. Men and Masculinities. DOI: 10.1177/1097184X18816118

Vetlesen, A. J. (2005). Evil and human agency: Understanding collective evildoing. Cambridge University Press.

Vito, C., Admire, A., \& Hughes, E. (2018). Masculinity, aggrieved entitlement, and violence: considering the Isla Vista mass shooting. NORMA, 13(2), 86-102. DOI: $10.1080 / 18902138.2017 .1390658$

Waling, A. (2019). Rethinking masculinity studies: Feminism, masculinity, and poststructural accounts of agency and emotional reflexivity. The Journal of Men's Studies, 27(1), 89-107. DOI: $10.1177 / 1060826518782980$

Wardlaw, G. (1989). Political Terrorism: Theory, Tactics and Counter-Measures. Cambridge: Cambridge University Press.

Wendling, M. (2018). Alt-right: From 4chan to the White House. Pluto Press.

Westlake, B. G., \& Bouchard, M. (2016). Liking and hyperlinking: Community detection in online child sexual exploitation networks. Social science research, 59, 23-36. DOI: 10.1016/j.ssresearch.2016.04.010

Whitehead, S. M. (2002). Men and masculinities: Key themes and new directions. Cambridge: Polity.

Williams, Z. (2018). 'Raw hatred': why the 'incel' movement terrories women. Retrieved from https://www.theguardian.com/world/2018/apr/25/raw-hatred-why-incel-movement-targetsterrorises-women 
Winter, A. (2019). Online hate: from the far-right to the 'alt-right'and from the margins to the mainstream. In Harmer, E., \& Lumsden, K. Online Othering (pp. 39-63). Palgrave Macmillan, Cham.

World Health Organisation. (2014). Global status report on violence prevention, 2014. Luxemburg: World Health Organisation.

Yar, M. (2012) Crime, media and the will-to-representation: Reconsidering relationships in the new media age. Crime, media, culture 8(3) 245-260. DOI: 10.1177/1741659012443227

Yar, M. (2013). Cybercrime and the internet: an introduction. Cybercrime and Society, 2nd edn. London: Sage, 1-20.

Zimmerman, S., Ryan, L., \& Duriesmith, D. (2018). Recognizing the Violent Extremist Ideology of 'Incels'. Women In International Security POLICYbrief, 9, 1-5. 